\title{
Mechanosensing and Symmetry of Potassium Channels Studied by Molecular Dynamics Simulations
}

\author{
Dissertation \\ for the award of the degree \\ "Doctor rerum naturalium" \\ of the Georg-August-Universität Göttingen \\ within the \\ International Max-Planck Research School \\ for Physics of Biological and Complex Systems \\ of the \\ Göttingen Graduate School for \\ Neurosciences, Biophysics and Molecular Biosciences \\ submitted by \\ Julian Tim Brennecke \\ from Hildesheim
}

Göttingen, 2018 


\section{Thesis Committee Members:}

Prof. Dr. Bert de Groot (Reviewer)

Max Planck Institute for Biophysical Chemistry, Göttingen

Prof. Dr. Marcus Müller (Reviewer)

Georg August University, Göttingen

Prof. Dr. Luis Pardo

Max Planck Institute for Experimental Medicine, Göttingen

\section{Further Members of the Examination Board:}

Prof. Dr. Andreas Janshoff

Georg August University, Göttingen

Prof. Dr. Sarah Köster

Georg August University, Göttingen

Dr. Johannes Söding

Max Planck Institute for Biophysical Chemistry, Göttingen

Date of the disputation: October 2nd, 2018 


\section{Abstract}

Potassium channels are relevant in a variety of physiological processes in all three kingdoms of life. In mammals these processes including nerve signaling, heartbeat regulation, and osmotic regulation of cells. Thus a malfunction of these channels is the cause for multiple diseases making potassium channels an important target for drug design.

Some of these potassium channels are mechanogated. This particular group and their gating mechanism, as well as quantification of asymmetry in homomultimeric proteins, are the focus of this thesis. Mechanogating is the response of channels to membrane stress through bending and stretching and is involved in many physiological processes including the sensation of touch, hearing, and pain perception. Crystal structures of mechanogated potassium channels can, in some cases, be directly interpreted as open (conductive) or closed (non conductive) states and the gating mechanism can directly be inferred. However, other channels of this group display different states as observed by crystallographic structure determination, but their configuration does not permit insight to the underlying gating mechanism. Two of these channels are the mechanogated two-pore domain potassium channels TREK-2 and TRAAK which are studied here. To elucidate their gating and mechanogating mechanism in particular, we applied molecular dynamics (MD) simulations, allowing insight down to atomistic scale.

First we studied the hypothesis of the lipid block based gating mechanism, that suggests a lipid entering the channel blocking the ion permeation pathway. Our results challenge this mechanism as we find ion permeation even in the presence of the lipid that is proposed to be responsible for the lipid block. We suggest a gate located in the narrowest region of the channel called the selectivity filter. The open probability of this gate is influenced by the states of the channel. Two states are known from crystallographic structure determination termed 'up' and 'down'. We find the up state increasing the open probability compared to the down state. As our main interest is on mechanogating of these channels, we varied the membrane tension to trigger this mechanism. Corresponding MD simulations display a transition towards the up state at increased membrane tension and by this the open probability is increased as corroborated by experimental results. Concluding this research we formulated an overall model by which the channel states as known from crystal structures influence selectivity filter stability and are influenced by membrane tension.

Second we studied asymmetry of homomultimeric proteins of which potassium channels are a typical representative. Many computational analysis tools implic- 
itly use ensemble averages to determine protein motions, e.g. Principal Component Analysis, and thus neglect asymmetry which can be important for protein function. Therefore, a solid understanding of asymmetry is required to correct for the neglect. A first step towards this aim is asymmetry quantification. To do so, we developed two algorithms, one evaluating the overall asymmetry and a second one restricted to a functional reference motion. We applied the algorithms to modified potassium channels TREK-2 and KcsA to demonstrate their ability to correctly quantify asymmetry. Furthermore, we applied it to the unfolding process of TTR demonstrating their ability to handle even complex functional properties. Both algorithms can therefore be used to quantify the asymmetry of homomultimeric proteins.

In this thesis we provide evidence for a selectivity filter based gating mechanism and demonstrate how this mechanism can be influenced by membrane tension. Furthermore, we provide two algorithms able to quantify asymmetry and demonstrate their usage. 


\section{Acknowledgment}

At first I would like to thank my supervisor Prof. Bert de Groot for the opportunity to do my $\mathrm{PhD}$ in his group. His guidance and patience made this thesis possible. I appreciate him giving me the chance to try different things and follow my own path even so it sometimes was not completed with successfully results. Special thanks to Petra Kellers for reviewing and improving my manuscripts. Further, I would like to thank GGNB and its representatives for the opportunities the program provided, from conferences to retreats and interesting courses. This added an additional layer to the $\mathrm{PhD}$ research and opened up the chance to meet other PhD students from different labs.

GGNB also required a thesis committee which lead to three interesting meetings with Prof. Marcus Müller and Prof. Luis Pardo. I am especially thankful for their outside view, creative ideas and assurance of help in case of any problems. It was a great benefit which I appreciate.

I want to thank all present and former department members for creating an environment of support and cooperation. This created the chance to profit from each other. Apart from personal discussions, I learned at least as much from seminars of others about biological systems and techniques as from my own studies.

I also want to thank the DFG for funding my position as part of the SFB 803.

Last but not least I want to thank my family. My wife as well as my parents supported me during the entire time of the thesis. They suffered the most from my bad mood if things did not work as planed. But they always encouraged me to look ahead and press on. Without them, this time would have been much harder. 


\section{Contents}

1. Introduction 1

1.1. K2P Channels . . . . . . . . . . . . . . . . . . . . . 2

1.2. Evolutionary and Physiological Role of Mechanogating . . . . . . . 5

1.3. Principles of Mechanogating . . . . . . . . . . . . . . . 6

1.4. Electrophysiology . . . . . . . . . . . . . . . . . . . . . 8

$\begin{array}{ll}\text { 2. Aim and Organization of the Thesis } & 11\end{array}$

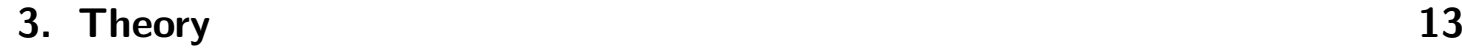

3.1. Molecular Dynamics Simulations . . . . . . . . . . . . . . . . . . . 13

3.2. Computational Electrophysiology . . . . . . . . . . . . . . . . . . . 19

3.3. External Electric Field . . . . . . . . . . . . . . . . . . . . . . . . . 20

3.4. Membrane Tension Coupling . . . . . . . . . . . . . . . . . . . . 21

3.5. PLS-FMA . . . . . . . . . . . . . . . . . . . . . 21

4. Gating by Lipid Block 23

4.1. Introduction . . . . . . . . . . . . . . . . . . . . 23

4.2. Methods . . . . . . . . . . . . . . . . . 25

4.3. Results . . . . . . . . . . . . . . . . . . 26

4.4. Discussion \& Conclusions . . . . . . . . . . . . . . . . . . . . 30

5. Mechanosensitive Gating of TREK-2 33

5.1. Introduction . . . . . . . . . . . . . . . 33

5.2. Methods . . . . . . . . . . . . . . . . . 36

5.3. Results and Discussion . . . . . . . . . . . . . . . . . . 37

5.4. Conclusions . . . . . . . . . . . . . . . . 44

5.5. Author Contributions . . . . . . . . . . . . . . . . 46

5.6. Acknowldegments . . . . . . . . . . . . . . . . 46 
$\begin{array}{lr}\text { 6. Selectivity Filter Exchange } & 47\end{array}$

6.1. Introduction . . . . . . . . . . . . . . . . . . 47

6.2. Methods . . . . . . . . . . . . . . . . . . . . . 48

$6.3 . \quad$ Results . . . . . . . . . . . . . . . . . . . . . . . . . . . 49

6.4. Discussion \& Conclusions . . . . . . . . . . . . . . . . 51

7. Quantifying Asymmetry of Multimeric Proteins 57

7.1. Introduction . . . . . . . . . . . . . . 58

7.2. Theory . . . . . . . . . . . . . . . . . . 60 60

7.3. Results . . . . . . . . . . . . . . . . . . . . . . . . 65

7.4. Discussion . . . . . . . . . . . . . . . . . . . . . . . . . . . 69

7.5. Conclusions . . . . . . . . . . . . . . . . . . . . 71

7.6. Acknowledgment . . . . . . . . . . . . . . . . 71

\begin{tabular}{ll}
\hline 8. Summary and Conclusions & $\mathbf{7 3}$
\end{tabular}

8.1. Gating and Mechanogating. . . . . . . . . . . . . . . . . . . . . 73

8.2. Quantification of Asymmetry . . . . . . . . . . . . . . . . 74

\begin{tabular}{ll}
\hline 9. Outlook & 77
\end{tabular}

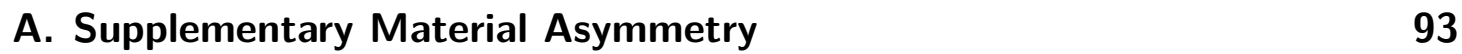

A.1. User Manual Asympy . . . . . . . . . . . . . . . . . . . . . . . . . . 93

A.2. Supplementary Figures . . . . . . . . . . . . . . . . . . . . . . 97 


\section{Chapter 1}

\section{Introduction}

Potassium channels are membrane-spanning proteins, conducting potassium ions $\left(\mathrm{K}^{+}\right)$across the cell membrane at a rate of $10^{6}$ to $10^{8}$ ions/s. These proteins are found in excitable as well as non-excitable cells and can be linked to various functions. Some of these functions are the regulation of neurotransmitter release, heart rate control, insulin secretion, neuronal excitability, epithelial electrolyte transport, smooth muscle contraction and cell volume regulation [1]. Whenever a protein is functionally important, its dysfunction can lead to serious diseases. For $\mathrm{K}^{+}$channels these include diseases of the heart [2], kidney [3], pancreas [4] and the central nervous system [5]. The majority of these diseases is linked to gene mutations of varying potassium channels. For a more detailed overview on potassium channel pharmacology we refer to the review of Shieh et al. [1].

The structure of potassium channels is variable. However, common motifs can be found in any potassium channel. The first common feature of all potassium channels is the selectivity filter. This specific region forms the narrowest part of the permeation pathway and selectively conducts potassium ions. An additional feature all potassium channels share is their ability to gate [6]. Gating refers to the fact that at least two states of these channels are known which are the open and closed state with high and no or little ion conductance, respectively. However, triggers opening and closing the channel vary greatly between the different types and include $\mathrm{pH}$, temperature, membrane tension, transmembrane voltage and ligand binding 70 . 10 .

asdfa Potassium channels can be distinguished by the structure of the pore-containing subunits [1]. Following this classification three groups of $\mathrm{K}^{+}$channels can be identified. The first group includes the voltage-gated (Shaker-like) potassium channels. These consist of four subunits, each containing one pore region and six regions of transmembrane helices of which four form a voltage sensor. Each subunit contributes one pore region to form the selectivity filter at the symmetry axis. The second group comprises the inward rectifying $\mathrm{K}^{+}$channels composed of two trans- 
membrane regions and a single pore region per subunit. The third group covers the two-pore (domain) potassium channels. In contrast to the other groups, these are only assembled of two similar subunits and therefore present only a pseudofourfold symmetry rather than a strict fourfold symmetry. As the selectivity filter shares high similarity between all potassium channels, two-pore channel subunits contribute two pore regions each to the selectivity filter (see figure 1.1) including typically four transmembrane domains.

As two-pore (domain) potassium channels (K2P) are in the focus of the research presented in this thesis, the following section will introduce this particular family of potassium channels in more detail.

\subsection{K2P Channels}

Similar to other potassium channels, members of the two-pore domain (K2P) potassium channel family are found in a variety of excitable and non-excitable cells. The typical structure of these family members is illustrated in figure 1.1. K2P channels are homodimers: they consist of two identical subunits. Both subunits share four (relevant) structural motifs. The first motif consists of transmembrane helices. Channels of the K2P family have amino acids arranged in four $\alpha$-helices spanning the membrane from the cytoplasmic to the extracellular side. Additionally, a CAP domain of helices can be found on the extracellular side. The most important part of the channel consist of residues contributing to the selectivity filter. In contrast to typical fourfold symmetric potassium channels, K2P channels contribute two pore regions per subunit to the selectivity filter, creating a unique pseudo fourfold symmetry. The last important motif in K2P channels consist of the pore helices. These are two helices per subunit which span only half the membrane sitting between the selectivity filter and the transmembrane helices stabilizing the selectivity filter. An additional structural feature can be found in the K2P subfamily TRAAK/TREK that is a C-terminal domain reaching into the cytoplasmic side. However, no structural information is available on this domain as x-ray crystallographic studies or other structure determination experiments so far do not include this region.

Physiologically, K2P channels are thought to create a so called 'leak' or 'background' current that stabilizes the resting membrane potential [11]. Even though K2P channels are considered as 'leak' channels, they were identified as important clinical targets for the treatment of cardiovascular diseases and several neurological disorders including pain and depression 11.

The K2P channel family encompasses 15 channels; three of them show stretch activation. As the main focus of this thesis will be on mechanogating, these three channels are of major importance. They are termed TRAAK, TREK-1 and TREK-2 


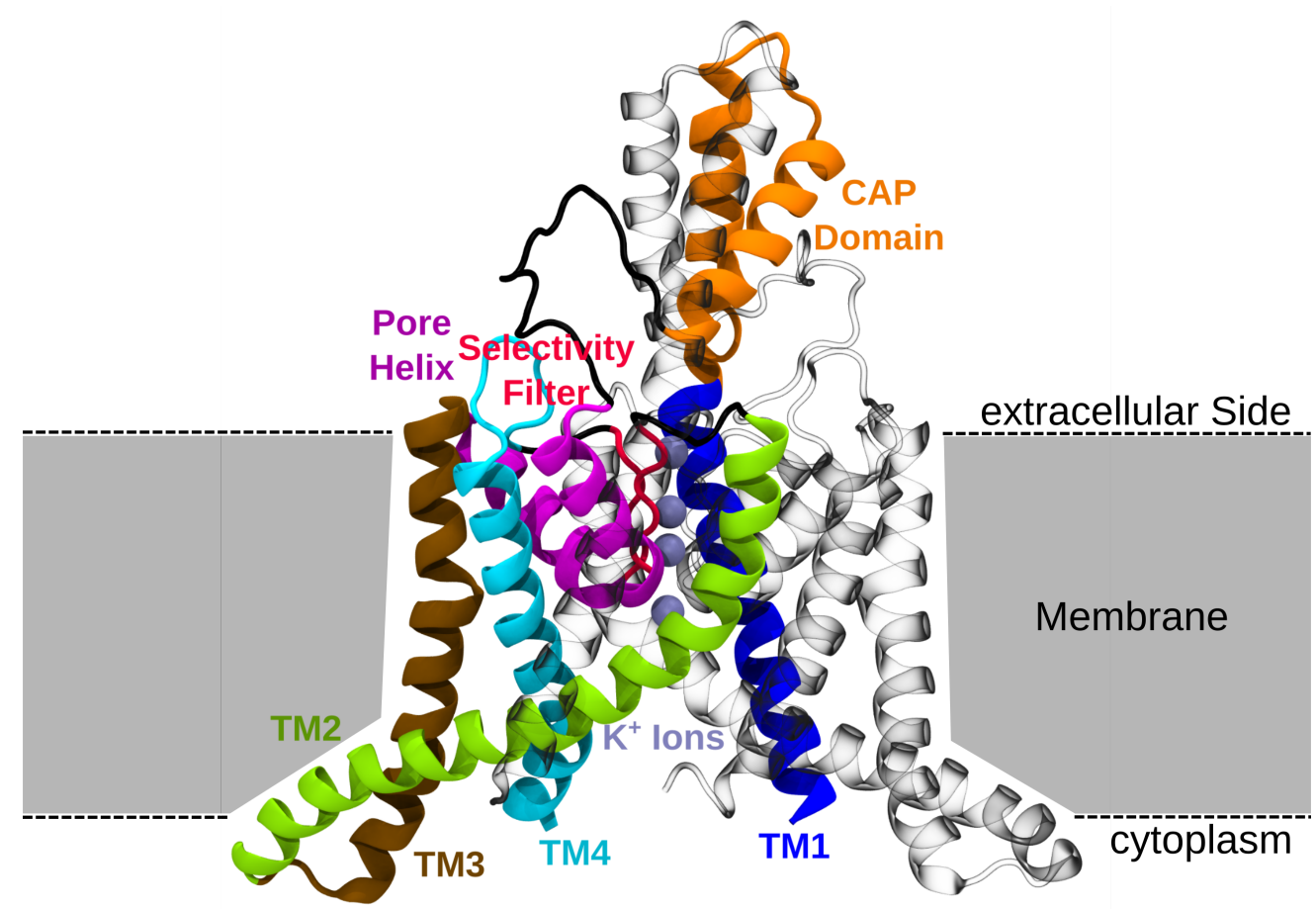

Figure 1.1.: Structure of K2P channels K2P channels are homodimeric potassium channels consisting of four transmembrane $\alpha$-helices (TM1-4). On top of the channel sits the cap domain (orange $\alpha$-helix). The selectivity filter (red) shows the typical fourfold symmetry of potassium channels and is the narrowest for the ions (ice blue spheres) to pass. Behind the selectivity filter sits the pore helix (raspberry) stabilizing it. The part of the protein located inside the membrane is marked with gray background. 


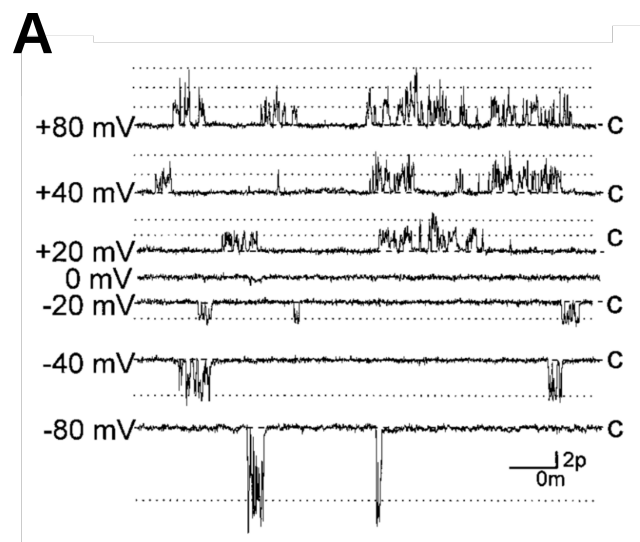

B

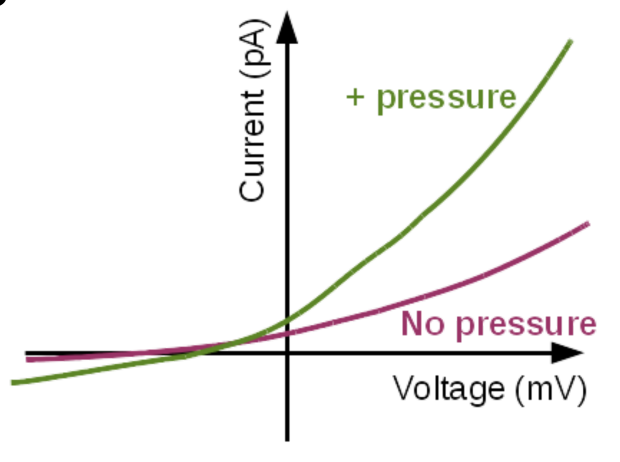

Figure 1.2.: Gating of mechanosensitive K2P channels A Single channel recordings show the open and closed state (c) of TREK-2 at different transmembrane voltages [8]. B These recordings are translated to IV curves, characterizing the behavior of ion channels. They show increased current if membrane pressure is applied.

and thus the subfamily is called the TRAAK/TREK subfamily. All three channels show a two to four fold increased current as a response to $-50 \mathrm{mmHg}$ applied pressure (refer to section 1.4 for experimental details) 12.

A prerequisite for molecular dynamics simulations is the availability of high resolution crystal structures. The crystal structures for TRAAK and TREK-2 show two different states. As mentioned before, these crystal structures lack the C-terminal domain. Nevertheless, gating studies of C-terminal truncated TRAAK/TREK channels demonstrate a similar gating behavior as the wild type channel (see figure 1.2 making it an appropriate structure to study gating [12,13]. The two states were termed up and down as the TM4 helix is either tilted more upward or more downward.

Some contradicting speculations have been made on the conductance of the two states. Ion occupancy in the crystal structure is commonly used to infer the conductance of the crystallized state 14, 15. Following this argument, the up state is considered more conductive than the down state as it shows higher ion occupancy $12,13,16,17]$. This hypothesis is supported by inhibition studies of the TREK- 2 channel. In these the active metabolite norfluoxetine binds to the channel at physiological relevant concentrations and enforces the down state. The inhibitory properties of norfluoxetine suggest the down state to be non conductive [18, 19. However, other mutational studies suggest the down state to be the stretch-activated state across the TRAAK/TREK channel subfamily 20. As a result the question on the conductivity of the states remains unanswered. 
Another open question concerns the gating mechanism of K2P channels. Functional studies show a lack of the classical bundle crossing gate as commonly found in potassium channels, such as KcsA and thus support a selectivity filter gate $14,21,25]$. The bundle crossing gate requires close contact of the transmembrane helices of the different subunits thus sterically blocking ion permeation. In contrast, a selectivity filter gate could be similar to C-type inactivation as seen in KcsA ( [26]). In this mechanism, the selectivity filter changes its configuration to either allow ions to pass or block them. The stability of the selectivity is speculated to be controlled by the C-terminus that alters the TM4 and TM2 helices [27]. A complete understanding of $\mathrm{K} 2 \mathrm{P}$ gating is beyond the current state of research. With this thesis I aim to improve the understanding of the gating mechanism and especially the mechanogating of K2P channels.

\subsection{Evolutionary and Physiological Role of Mechanogat- ing}

One hypothesis on the functional role of ion channels in the early evolutionary stages of life suggests mechanogating to be their original function. In these early development states, the cell's outer shell was formed by two fatty acid leaflets $[28,29$. The metabolic conversion of sugar inside these cells created increased cytoplasmic osmolarity [30]. Consequently, water was pulled into the cells through the membrane 31]. Without any regulation of this process, the cells would burst and would be destroyed. To antagonize this effect, a release mechanism of metabolites was required to reduce osmolarity. A conceivable mechanism would be mechanosensitive channels acting as emergency release 32 . After the cell has taken up water, the cell membrane got stretched. This stretching is the activation mechanism of mechanosensitive channels that in turn release metabolites such as ions from the cell. This hypothesis arises from the idea that all ion channels are intrinsically mechanosensitive 33. However, the majority of ion channels probably lost their mechanosensitive function during evolution 34 .

Nowadays, mechanosensitive ion channels have evolved to cover a wide range of important physiological processes such as perception of touch, hearing, balance and the sensation of pain [35,36]. Touch perception is mediated by Merkel cells. These cells are squeezed by the touch creating a membrane tension. Specialized channels inside the cell react to the change in membrane tension and activate. The ion content of the cell surrounding is changed by the opening of the channels triggering nerve endings in the vicinity [37.

The mechanism of hearing is fundamentally different to touch sensation. In spe- 
Force From Lipids

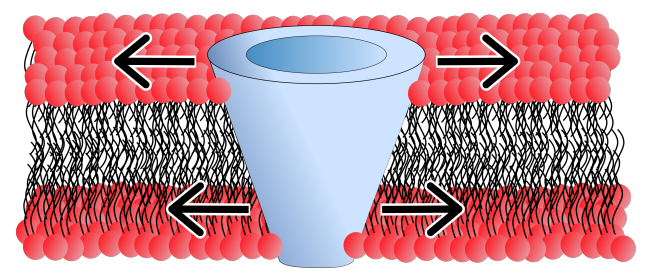

Tethers Attached

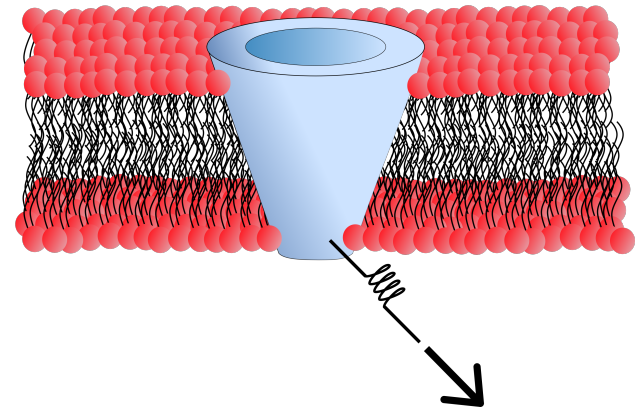

Figure 1.3.: Principles of Mechanogating Three major principles are known for mechanogating, the two most important are the force from lipids (FFL) principle and the tethers attached principle. Channels controlled by the force from lipid principle are directly gated by a change in membrane tension. This membrane tension pulls at the channel and leads to a change in its structure. In contrast, the tethers attached principle requires linkers to be attached to the channel. An example are the hair cells in the ear. Channels in the hair cells are connected by tethers to the next hair cell. At a relative motion of the cells towards each other, these tethers pull the channel open.

cialized hair cells, mechanosensitive channels are located at the distal tip of the stereocilia 38. So called tip links connect each stereocilium with its longer neighbor. These connections follow the tether attached principle (see next section) and open the channels upon movement. For hearing, mechanically activated cation currents as present in rodent sensory neurons are well described in biophysical terms 39 41]. But the molecular identity of mechanotransducer channels contributing to senses of touch and pain remain largely unknown 42.

Many eukaryotic mechanosensitive ion channels for these functions were identified including NOMPC [43, Piezo1 and Piezo2 [44] and TRP [45]. However, most of the understanding nowadays comes from mechanosensitivity of the bacterial channels MscL and MscS [32] which are structurally very different from eukaryotic channels. For a more extended overview we refer to the review of Árnadóttir et al. 46

\subsection{Principles of Mechanogating}

To date, three major mechanisms of mechanogating are known 1) G-Protein coupled, 2) Force From Lipids and 3) Tethers Attached. G-Protein coupled gating will not be considered in the following as it is indirect and not relevant for the thesis. The main idea is that a G-Protein coupled receptor is modified by mechanical 

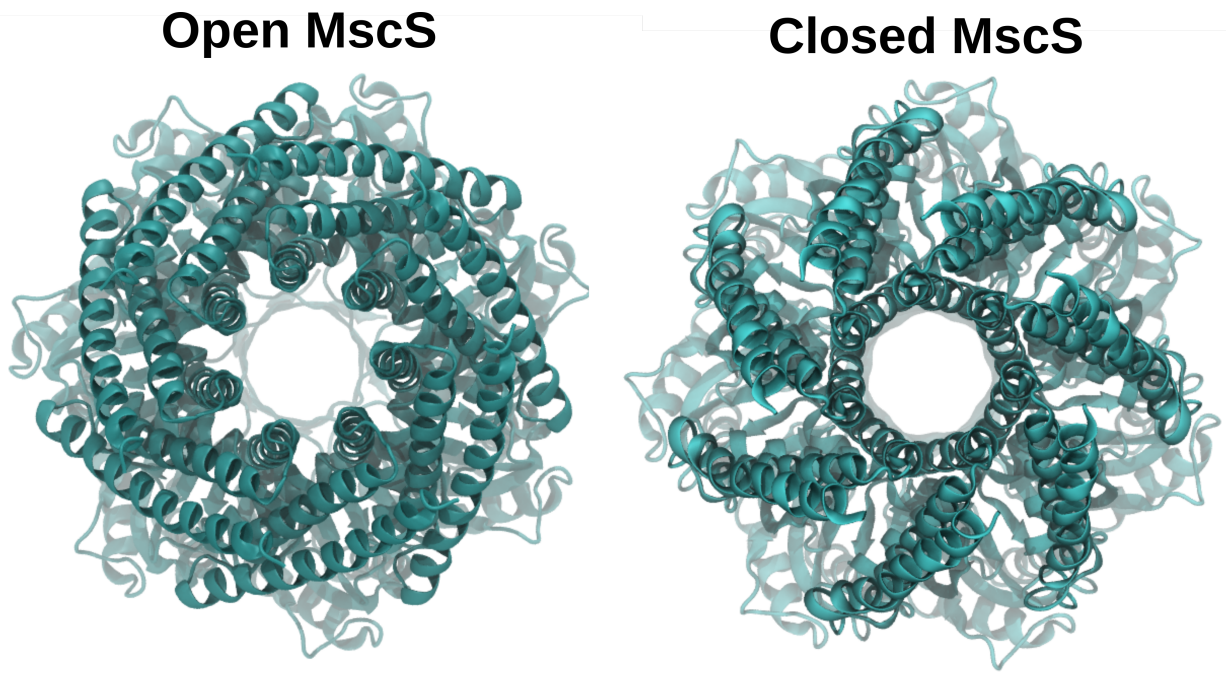

Figure 1.4.: MscS Gating Top view of the open and closed MscS channel (PDB ID open: 2VV5, closed: 2HW9). The open state shows a large pore were as the closed state sterically hinders ions from passing. The channel opens and closes by a tilt of the helices.

stress which regulates as a downstream process the activity of an ion channel.

The second mechanism is the "Force From Lipid" (FFL) principle (see figure 1.3 [47-49]. This principle states that the pressure inside the cell changes the membrane tension and thus directly influences the channel. As the channel sits inside the membrane, it can be affected by a change in membrane tension. A good example of this gating behavior is the bacterial MscS channel [50]. This channel is formed by helices spanning the membrane in a barrel like arrangement. As the membrane tension changes, the helices are pulled outward and tilt further into the membrane, creating a more open like structure (see figure 1.4). Due to the larger pore in the middle of the channel, the ions can pass more easily and the current through the channel increases.

The third principle is the "Tethers Attached" principle (see figure 1.3 B) [51]. Channels following this principle have a tether which is either connected to the cytoskeleton or to another structure as in the stereocilia in hair cells. If the channel changes its position relative to the structure it is attached to, the channel is pulled open or closed by the tether.

For the system we are interested in, namely the K2P channels, the FFL principle applies as pressure induced activation of TRAAK is enhanced upon cytoskeletal disruption or patch excision, suggesting a direct response of TRAAK to the physical state of the bilayer $[52]$. This concept is also assumed to be true for the other 


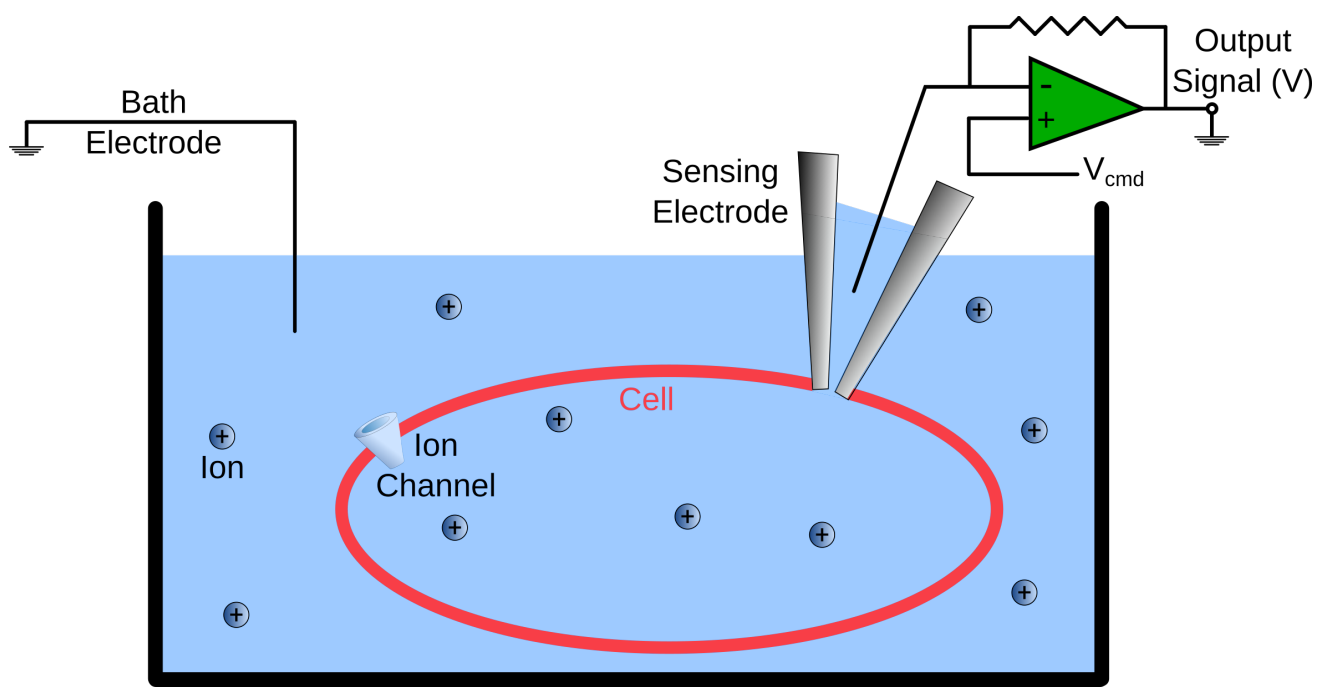

Figure 1.5.: Patch Clamp Electrophysiology Currents through ion channels embedded in a cell are measured by a voltage created by electrodes in- and outside of the cell. The current is used to characterize the channel.

members of the TREK/TRAAK subfamily.

\subsection{Electrophysiology}

Comparison to experimental data is required to validate the results of molecular dynamics (MD) simulations. Therefore, MD simulations are typically created to replicate the main components of experimental setups. In this section, the basic principles of relevant experimental setups to determine channel conductivity and mechanogating processes are presented.

An assay was developed to detect small electrical currents to be able to characterize the gating behavior of ion channels [53]. This method was termed patch clamp electrophyisology. In the typical whole cell patch clamp setup (see figure 1.5), an electrode-containing glass micropipette is placed at the surface membrane of an individual cell. By applying suction to the micropipette, the membrane enclosed by the pipette tip is removed. A second electrode inside the water bath outside the cell can now be used to apply a voltage difference between the in- and outside of the cell. This voltage difference drives ions across channels located in the membrane. Channels can be characterized by measuring the resulting current at different voltages in the picoampere range. The results of these measurements are so called IV (current against voltage) curves (see figure 1.2).

A major advantage of this technique is the high temporal resolution allowing to 


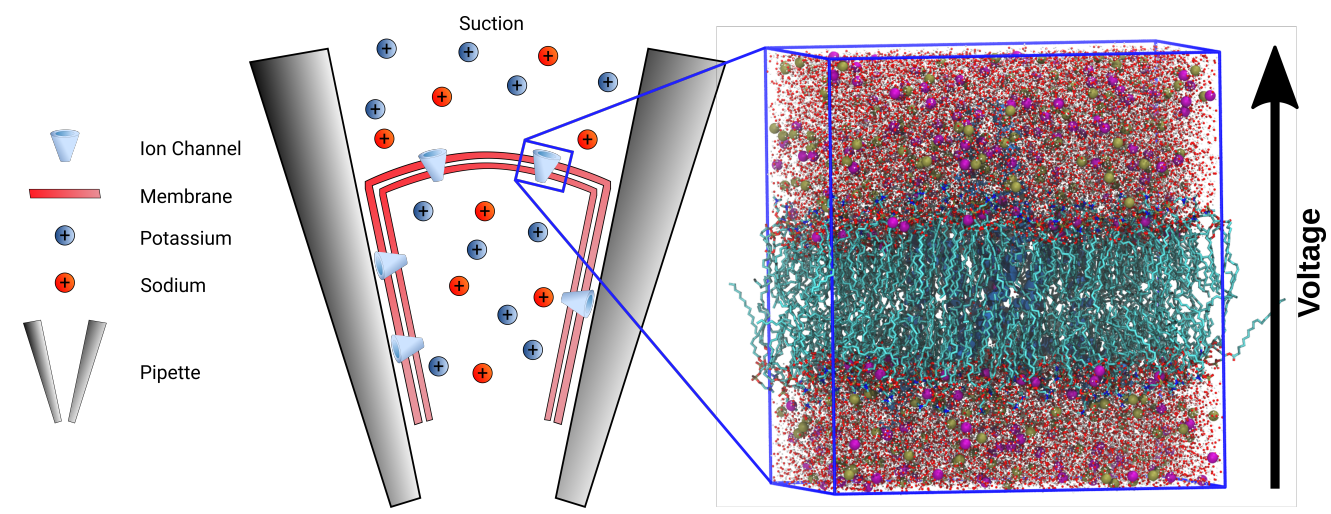

Figure 1.6.: Excised Patch Electrophysiology A pipette is used to excise a patch from a cell. Ion channels in the patch can be characterized by currents as a response to a transmembrane voltage. By sucking at the membrane, an additional membrane tension can be applied and therefore the pressure response can be measured. This setup is mimicked by the simulation setup (right inset).

determine the rapid kinetics of ion channels. Moreover, it can detect flow rates as low as $10^{7}$ ions per second. The technique is highly reliable as the readout is direct and the environment can be controlled. For a detailed review on ion channel characterization we refer to the review of Clare [54].

To study mechanogating of ion channels requires controlling the membrane tension as well as the transmembrane voltage. To achieve this level of control, excised patches can be used. To excise a patch, a micropipette is brought in contact with a cell membrane as in the whole cell setup. After a seal between the tip of the pipette and the membrane is formed, the pipette is removed from the cell while some suction is applied simultaneously. This results in the excision of a membrane patch which subsequently covers the tip of the pipette (see figure 1.6). IV curves can be recorded of these patches similar to the whole cell setup. In addition, suction can be applied inside the pipette what leads to a bending of the membrane in the upward direction. By this, the membrane is not only bent but also stretched, a possible second cause for mechanogating. However, for TREK/TRAAK channels, measurements bending the membrane in either direction were performed showing an independence of the direction in which the membrane was bent. This result hints at a direct tension response unrelated to membrane bending [12]. Taking geometrical considerations into account enables the quantification of the membrane tension due to suction. With the suction applied, IV curves can be determined once more, now also accounting for the increased membrane tension.

Thus we have two crystal structure states with an unclear functional readout and electrophysiology measurements showing two different conductance levels. How- 
ever, how the states are related to conductance levels remains unknown. 


\section{Chapter 2}

\section{Aim and Organization of the Thesis}

With this thesis I would like to contribute to the understanding of K2P channel gating on an atomistic level particularly focusing on the mechanogating mechansm of the TRAAK/TREK channel subfamily. At first, the relevant methods are introduced in chapter 3. These are first and foremost Molecular Dynamics (MD) simulations, the method to create data for analysis. However, to be able to compare the results of MD simulations to experiments, ion currents through channels have to be measured. The MD extensions Computational Electrophysiology and External Electric Fields are introduced. Both methods are different approaches to create a transmembrane voltage, the driver of ion permeation. Finally, PLS-FMA, a tool to calculate the motion with the highest correlation to a functional measure, is introduced. This method is the basis of an asymmetry measure introduced in chapter 7 .

The first results chapter (4) gives insight into the conductivity of selectivity filter states. This understanding is further improved in chapter 5 as it is partially contained in the publication underlying this chapter. In chapter 4 a lipid inside the channel is shown not to gate the channels of the TRAAK/TREK family as speculated by Brohawn et al. [16] In contrast, we find that the gating of these channels takes place in the selectivity filter.

Further analysis on the role of the selectivity filter in conductance of the channel structures was performed and is presented in chapter 5. The understanding enables us to study the mechanogating mechanism of the channel directly by applying membrane tension in MD simulations. As a result, structural transitions from a less conductive to a more conductive state are observed. In addition, the direct current measurement of the ion permeation shows that this transition effectively alters the conductance of the channel which compares well with experimental results.

In chapter 6, an exchange protocol of the selectivity filter is applied. It is demonstrated that the state of the selectivity filter mainly defines the conductance of the 
channel rather than the overall structure.

Existing methodologies would artificially classify the same motion in different subunits differently, overlooking that asymmetric conformational changes may be related by symmetry. Even more, averaging such motions by imposing symmetry, as in the case for e.g. PCA or related techniques, suppresses such asymmetric contributions, thereby possibly leading to the risk of overlooking functionally relevant motions. Thus to be able to deal with asymmetry, new methods are required. A first step towards understanding asymmetry is its quantification. In chapter 7 , an extension of two different methods are introduced which enable the quantification of asymmetry and makes the correction for asymmetry possible. These methods could be applied to K2P channel gating as the selectivity filter changes asymmetrically.

The thesis is rounded off in chapter 8 by a summary of all presented results and the conclusions we can draw from these results. Finally, an outlook is given hinting at further research opportunities identified by this research. 


\section{Chapter 3}

\section{Theory}

In this chapter, an introduction to the methods and techniques as employed in this thesis is given.

At first, molecular dynamics (MD) simulations are introduced. This computational method is the basis of all simulations to generate dynamic data of the studied proteins. To study permeation of ion channels in MD simulations, a technique to simulate transmembrane voltages is required. Therefore, two methods, namely Computational Electrophysiology (CompEL) and External Voltage Simulations, are introduced achieving a transmembrane voltage in two different ways. Finally, the algorithm PLS-FMA is introduced which is later used to classify the selectivity filter states as conductive and non conductive and is extended in chapter 7 to study asymmetry.

\subsection{Molecular Dynamics Simulations}

Various methods such as X-ray crystallography, FRET and AFM exist to study the structure and dynamics of biomolecules. However, all of these methods are limited in spatial as well as in temporal resolution [55. In figure 3.1 different techniques and their resolution are compared.

Another method to study the dynamics of proteins is the application of all-atom molecular dynamics (MD) simulations. MD simulations allow insight into atomic motions from a femtosecond to millisecond time scale. This short time scale makes MD suitable to complement experimental techniques by providing additional information. In the following the basis of MD simulations will be derived starting from quantum mechanics which is the dominant physics concept at atomistic length scales. The algorithms necessary to simulate a system as precisely as possible is introduced. At the end, an overview over the limitations of MD is given. 


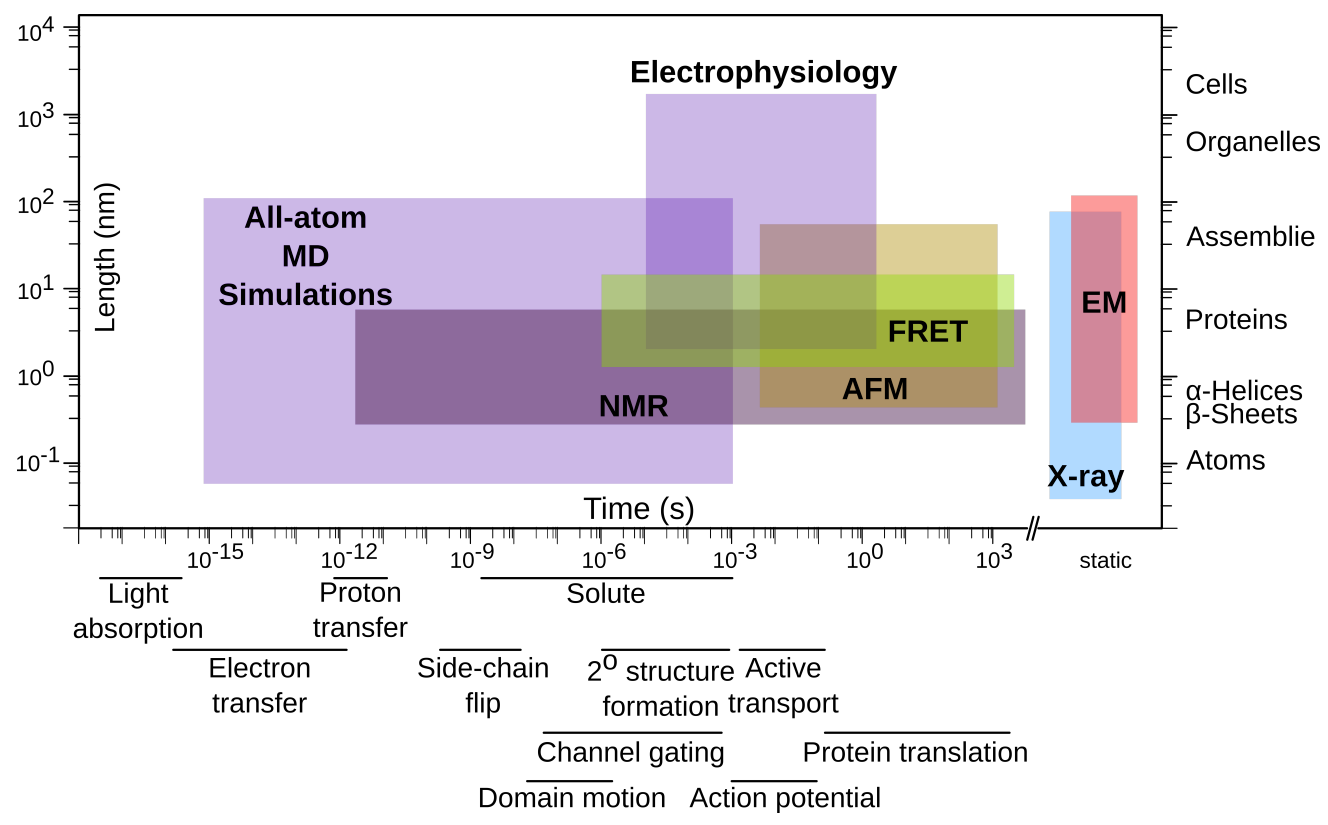

Figure 3.1.: Comparison of spatial and temporal resolution Various techniques are available to study biophysical processes. However every technique has limitations in spatial and temporal resolution. Processes on various time scales as well as representative objects on length scales are shown as a reference. (Figure based on [55].)

\subsubsection{From QM to MD}

As mentioned before, the dynamics at atomistic length scales are dominated by quantum mechanics (QM). The dynamics of any atomic system can be described by the Schrödinger equation. Solving the time dependent variant of the Schrödinger equation shows the time evolution of the system. However, as solving the Schrödinger equation is computationally demanding approximations are required to effectively simulate atoms.

Born-Oppenheimer Approximation The Schrödinger equation solves the wave function of the electrons and the nuclei simultaneously. However, as Max Born and J. Robert Oppenheimer found, the complexity of the problem can be reduced: electrons are much lighter than nuclei. As a result, the motion of nuclei can be treated as resting compared to the motion of an electron. Therefore, the motions of electrons and nuclei can be separated and thus the wave functions can be solved independently.

To study the motion of biomolecules, the motion of the nuclei is most relevant. To get the motion of each nucleus, the electron wave function and the force acting on 
the nucleus is calculated. These forces allow updating the positions of the nuclei. A method using this approach to calculate motions based on QM is named $a b$ initio MD [56].

A major drawback of ab initio MD simulations is the massive computational effort which restricts the length of simulations to a few nanoseconds. These short time scales suffice to simulate fast processes. However, many biological processes involve major conformational changes which can not be sampled in such a short time. Consequently, an additional approximation is required increase the length of simulations.

Classical Nuclear Dynamics In $a b$ initio MD, the nuclei are described quantum mechanically. QM allows to simulate effects such as tunneling of light nuclei and high frequency vibrations of covalent bonds. If the tunnel effect is neglected and the vibrational modes are restrained, a classical

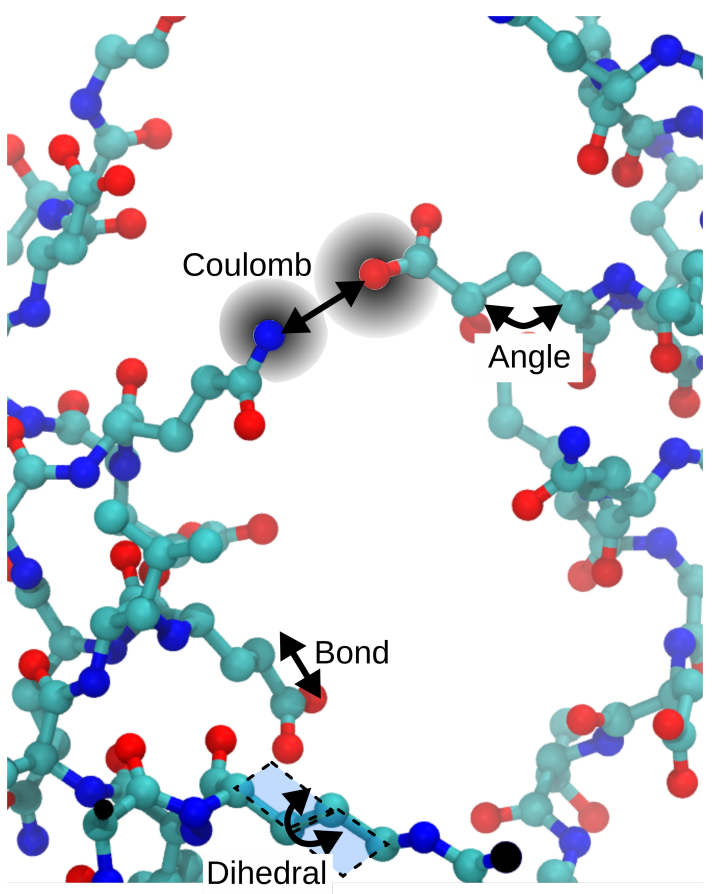

Figure 3.2.: Interactions Illustration of the different interactions. description of the nuclei can be used.

Force Field Approximation To calculate the forces on these classical nuclei more efficiently, calculation of the Schrödinger equation can be abandoned by defining an effective potential which describes the force between the atoms. The potentials depend on the atom and its environment. All potentials required to describe the forces acting on the atoms can be split into bonded and non-bonded interactions

$$
\begin{aligned}
V(x) & =\sum_{i} V_{i} \\
& =V_{\text {bonded }}+V_{\text {non-bonded }} .
\end{aligned}
$$

Bonded interactions are interactions of atoms which are covalently bound. These interactions can be described by a harmonic potential

$$
V_{\text {bonded }}=\frac{1}{2} \kappa\left(x-x_{0}\right)^{2} .
$$


In this equation, $x_{0}$ denotes the equilibrium conformation. If the atoms are not found in their equilibrium conformation, the spring constant $\kappa$ creates a force towards the equilibrium. The values for $x_{0}$ and $\kappa$ have to be defined. Three types of bonded potentials are used: bond, angle and dihedral potentials (see figure 3.2.

Bond potentials represent the force of two atoms connected by a chemical bond. However, in most cases the bond potentials are neglected in favor of a distance constraint. The algorithms used to impare the distance constraints are LINCS [57] or SHAKE [58].

Angle potentials are assigned to three atoms which are connected. The correct angle is assumed to be the smallest angle between the outer atoms which are connected to the central atom.

(Improper) dihedral potentials describe the interaction between four connected atoms. Dihedrals can be described by two planes and their orientation towards each other. These planes are defined by three atoms each. Either the first or last atom is excluded. The angle is defined to be zero in the cis configuration. An additional variant is the improper dihedral potential. This potential can also be applied to more complex arrangements of four connected atoms such as a central one with three atoms connected to it. The improper variant of this potential is especially relevant to describe planar groups such as benzol rings.

Non-bonded interactions act in contrast to the bonded interactions not only between covalently bound atoms but between all atoms within a certain distance. The calculation of these interactions is especially challenging for simulations as the atoms within a certain distance can change during the course of a simulation. To improve performance, a neighbor list is created with all atoms within a certain cutoff and is updated only after a predefined number of steps. Beyond a certain cutoff, most of the interactions are set to zero as also typically done for interactions between the first three bonded neighbors. The relevant non-bonded interactions are Lennard-Jones potentials and Coulomb potentials.

The Lennard-Jones potential consists of two parts

$$
V_{L J}=\frac{c_{1}}{r^{12}}-\frac{c_{2}}{r^{6}}
$$

The first part is the repulsive van der Waals term and is represented as $1 / r^{12}$. This term approximates the Pauli principle. The other part is an attractive $1 / r^{6}$ term which models the van der Waals interaction originating from the polarisability of the electron cloud around the atom. The parameters $c_{1}$ and $c_{2}$ differ between atom pairs and have to be determined.

The Coulomb potential describes the electrostatic interaction of atoms. In prin- 
ciple, the Coulomb potential can be calculated directly. However, as the potential only decays with $1 / r$, it is a very long ranged potential. Therefore, defining a cutoff distance can result in artifacts.

As a solution to the previously described problem, the periodicity of the system is used in modern MD simulation software. It allows the transformation into Fourier space and the Particle Mesh Ewald [59] (PME) method can be applied. PME reduces the complexity of the Coulomb potential interactions in a system with $N$ atoms from a $\mathcal{O}\left(N^{2}\right)$ problem to a $\mathcal{O}(N \times \log N)$ problem.

The above mentioned parameters are summarized technically in so called forcefields (FF). There are various FF differing in the actual parameters. These parameters are derived in different ways: Most FF use QM simulations as a starting point to derive the parameters that are later compared to experimental values and improved to reproduce experimental results. For the FF, different experiments are used against which they are evaluated. Thus to reproduce a physiological property of a system, some FF are more suited than others 60.

\subsubsection{Integrator}

Forces can be derived from the given potentials by calculating the spatial derivatives. These forces act on the nuclei resulting in motion of the atoms. As the system can be described as a classical system, Newton's equation of motion applies

$$
m_{i} \frac{\partial^{2} \vec{r}_{i}}{\partial t^{2}}=\vec{F}_{i}
$$

The force acting on any atom $i$ equals the mass $m$ times acceleration of the atom. This differential equation can now be integrated numerically. In MD simulations the leap frog algorithm is widely used as it is symplectic. The leap frog algorithm calculates the velocity at half steps and the atom positions at full steps

$$
\begin{aligned}
& \vec{v}_{t+\frac{\Delta t}{2}}=\vec{v}_{t-\frac{\Delta t}{2}}+\frac{\vec{F}_{t}}{m} \Delta t \\
& \vec{r}_{t+\Delta t}=\vec{r}_{t}+\vec{v}_{t+\frac{\Delta t}{2}} \Delta t .
\end{aligned}
$$

As the forces of the system $(\vec{F})$ are only calculated at any discrete time $(t)$, the selected time step $(\Delta t)$ has to be appropriate. Otherwise, the results would be inaccurate for too long time steps, due to the dependence of forces on atom positions and the large displacement of atoms during the time step. Neither can the time step be chosen too small as too many steps would be required to achieve 
a reasonable amount of simulation time. In general it was found that $1 \mathrm{fs}$ is a reasonable time step. However, by constraining the fastest motion, the hydrogen bond vibrations, the time step can be increased to $2 \mathrm{fs}$ while keeping the accuracy. In some cases, hydrogens can be replaced by virtual sites where they are represented as dummies and thus are fixed to the heavy atom they are bonded to. This replacement allows even for a $4 \mathrm{fs}$ time step [61].

\subsubsection{Periodic Boundary Conditions}

As the number of atoms which can be simulated is finite, the system inevitably will have boundaries. To prevent artifacts from these boundaries they are treated periodically. Periodic boundary conditions mean that at any boundary of the system a copy of itself is located creating an infinite environment. As mentioned before, periodic boundary conditions are commonly used in MD simulations. Periodic boundary conditions do not only allow for the use of Particle Mesh Ewald but also make the simulation more physical.

\subsubsection{Barostat and Thermostat}

MD simulations mimic conditions of a physiological environment. A typical environment is any type of cell. In a cell, the pressure and temperature are constant over time due to the large size of the overall system compared to proteins. Looking at one protein inside this cell and creating a box around it requires additional algorithms to keep a constant pressure as well as a constant temperature in the box (PT ensemble). Integration errors will otherwise lead to deviations from the initially set temperature. This is the system we are going to simulate. Furthermore, it is reasonable to keep the number of atoms inside this box constant. Thus we get an isothermal-isobaric ensemble (NPT). The NPT ensemble is achieved by coupling the system to an external heat and pressure bath. To keep the pressure constant, the box size is varied for example by the Parinello-Rahman barostat 62 . As the temperature of a system is the kinetic energy of the atoms, it can be manipulated by changing the velocities. The correct change in velocities is typically achieved by using the velocity rescaling algorithm [63].

\subsubsection{Limitations}

Due to the approximations made by neglecting QM in favor of a classical description of the forces, processes such as the formation and breaking of bonds are impossible to simulate in MD without additional algorithms. If this kind of processes are of interest in the studied system, methods combining QM and MD are available [64]. These methods simulate the majority of the system as MD whereas 
only the part relevant for the bond change is simulated as QM.

Furthermore, the current FF do not incorporate the polarizability of electron clouds. The electrostatics of atoms can influence each other which leads to an induced dipole which can be represented by polarizable FF [65-67]. Even though the development of these made great progress in the last years, it is not standard yet to use this type of FF.

Apart from this, FF only approximate the correct QM solution. This means that the accuracy of FF is limited. Another limitation that similarly applies to QM simulations is the numerical error in the integration. This error is the result of the discretization of the system and can be minimized by optimizing the time step. Large supercomputers can reach simulation times of multiple milliseconds. Nevertheless, many biological processes, even inside average sized proteins, are often unaccessible with MD simulations.

With the limitations of MD simulations in mind, it is useful to compare the results of any simulation against experimental data. This comparison should give a first hint if the simulated system is behaving in a physiological way. Furthermore, results of simulations may also suggest experimentally testable hypotheses.

\subsection{Computational Elec- trophysiology}

To study ion permeation through ion channels, we need a setup with an ion channel embedded in the membrane. In a voltage free simulation, the channel is very unlikely to perform any significant number of spontaneous permeation events. In nature, the permeations are driven through the difference in ion concentration inside and outside
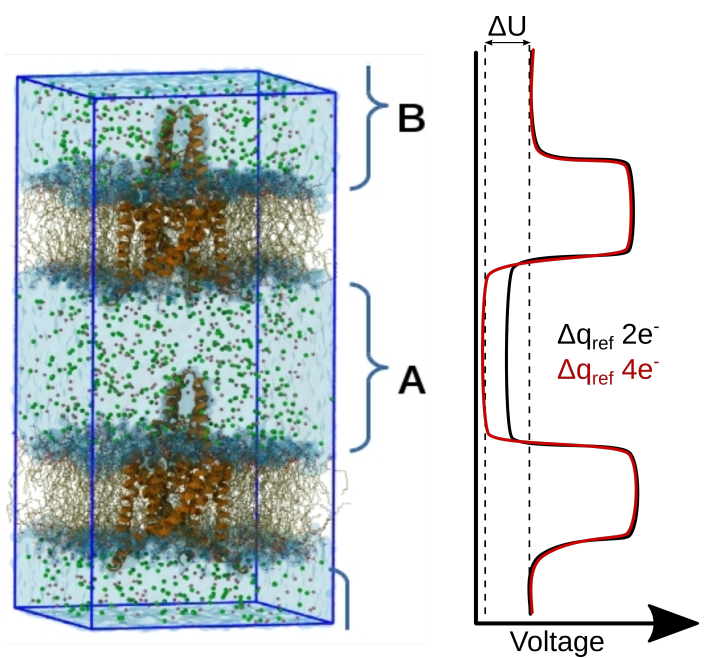

Figure 3.3.: Computational Electrophysiology A simulation box with the membrane and an ion channel inside is duplicated to get two compartments A and $\mathrm{B}$. By changing the number of ions in the different compartments, an ion gradient can be generated and kept constant by applying the Computational Electrophysiology concept. If the total charge of the inner and outer compartments differ it results in a transmembrane voltage (shown at the right). of the cell. The concentration gradient can be imitated in simulations by the Computational Electrophysiology approach to drive permeations in the most physiological way [68]. 
The basic setup can be seen in figure 3.3. The simulation system is split into two equally sized compartments separated by two membranes. An ion channel is located in each of the membranes. The two compartments allow for an ionic concentration gradient between the two compartments. As the electric potential is the double integrated ion density, a transmembrane voltage is introduced. Over time, the ion difference between the two compartments would deplete due to permeations through the channel. The Computational Electrophysiology algorithm uses an exchange between ions and water molecules to sustain the difference.

\subsection{External Electric Field}

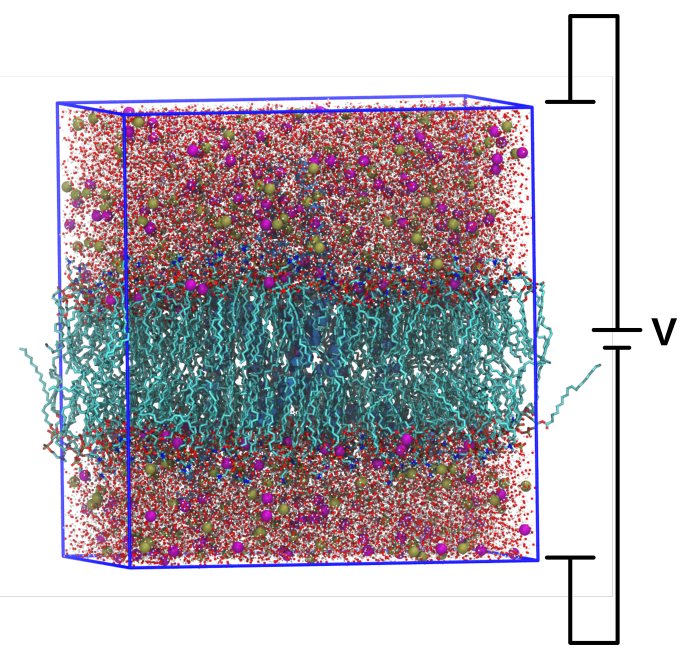

Figure 3.4.: External Electric Field In this algorithm, an external force is introduced that creates the effect of a transmembrane potential.
The external electric field is an alternative approach to simulate a transmembrane potential apart from the Computational Electrophyisiology approach. Here, a force is added to the ions similar to the force created by an electrical field. Roux 69 showed that the external electric field is a valid representation of the experimental setup where a voltage is created between two electrodes (see figure 3.4 ).

The Computational Electrophysiology approach as well as external electric field simulations are able to reproduce an electric field similar to experiments. Nevertheless, the two setups have different drawbacks and limitations. For the Computational Electrophysiology approach a larger box, usually twice the size of the electric field setup, is needed. Consequently, in a given real time, longer single trajectory simulations can be performed in external electric field simulations compared to Computational Electorphysiology simulations. In contrast, the relation between the external electric field and the locally acting field depends on the electrostatics scheme [70. Furthermore, in a system with a time dependent dielectric distribution, as in the case of a membrane pore formation, the external field has an unclear effect on the local electric field at the pore [71] and is therefore not suited for this type of systems. 


\subsection{Membrane Tension Coupling}

To study the effect of membrane tension on K2P channels we require control over it. The pressure is first corrected in z-direction using the standard barostat algorithm. Subsequently, the average membrane tension is calculated by

$$
\gamma(t)=\frac{L_{z}}{n}\left(P_{z z}(t)-\frac{P_{x x}(t)+P_{y y}(t)}{2}\right)
$$

where $L_{z}$ is the height of the box, $n$ the surface tension and $P_{x x}(t), P_{y y}(t)$ and $P_{z z}(t)$ the pressure in the corresponding direction. This membrane tension is then corrected towards a given reference value by varying the box length in $x / y$ direction.

\subsection{PLS-FMA}

After performing MD simulations, various tools are available to analyze the simulation data. One of these tools is the Partial Least Squares Functional Mode Analysis (PLS-FMA) analysis. This algorithm was extended during the scope of the thesis to incorporate asymmetry (see chapter 7).

PLS-FMA is a tool to identify a motion maximally correlated to a functional property of a system. This tool is required as the functional motion is hard to distinguish from the thermal fluctuations of the protein for many systems. Thus to use PLS-FMA, a property that is functionally relevant has to be characterized first. As an example, in an enzyme the distance between two residues at an active site could be used if it is known that this distance is relevant for its function. PLS-FMA searches for the motion which is maximally correlated to this distance. This motion is likely a motion relevant for the enzyme function.

In mathematical terms, PLS-FMA tries to predict the functional property $(f)$ from the trajectory $(\underline{X})$ by choosing optimal contributions $(\underline{b})$ of the different atoms to the functional property by reducing the squared sum of errors $(\underline{\epsilon})$ in the equation

$$
\underline{f}=\underline{b} \cdot \underline{\underline{X}}+\underline{\epsilon} .
$$

Due to the high dimensionality of $\underline{\underline{X}}$, a high risk of overfitting exists. To overcome this challenge the original implementation of FMA used a principal component analysis to reduce the dimensionallity $[72]$. Later the partial least squares (PLS) algorithm was used to reduce the dimensionality by choosing a limited number of PLS components 73]. Note that before using the PLS-FMA algorithm, the optimal number of components has to be identified by a cross-validation scheme 
(see 73 and chapter 7). 


\section{Chapter 4}

\section{Gating by Lipid Block}

This chapter focuses on the basic principles of TRAAK gating to prepare our further study of the mechanogating mechanism. Initially we considered the lipid gating hypothesis as proposed by Brohawn et al. [16], mainly based on a structure resolved by X-ray crystallography, to be the relevant gating mechanism. This hypothesis implies a lipid entering the cavity below the selectivity filter blocking ion permeation. In free simulations, a lipid partially entered the cavity possibly resembling a first step of this mechanism. To test if this lipid adopts the crystal structure configuration, we applied pulling simulations to drag the lipid towards the crystallographic configuration of the co-crystallized lipid. Simulations of this lipid inside the cavity demonstrate its inability to block the ion permeation pathway.

\subsection{Introduction}

As the gating mechanism of $\mathrm{K} 2 \mathrm{P}$ channels can not directly be deduced from the structure, as for example in $\mathrm{MscS}$, different gating hypotheses are under debate. Most experimental results suggest a selectivity filter gate as the most likely mechanism 21 24, 74]. What this gate may look like is discussed in more detail in chapter 5. However, some evidence hints at a lipid block mechanism as the TRAAK gating 16 .

The first hint pointing to the lipid block hypothesis came from X-ray crystallographic studies of TRAAK [16]. Two different states of the channel termed "up" and "down" state were identified. The ion occupancy of the selectivity filter suggests the down state to be a non conductive and the up state to be a conductive state. Furthermore, a lipid acyl chain accessing the lipid cavity below the selectivity filter was co-crystallized (see figure 4.1). This lipid entered the cavity by a fenestration created by the TM2 and the TM4 helix. As the name down state 


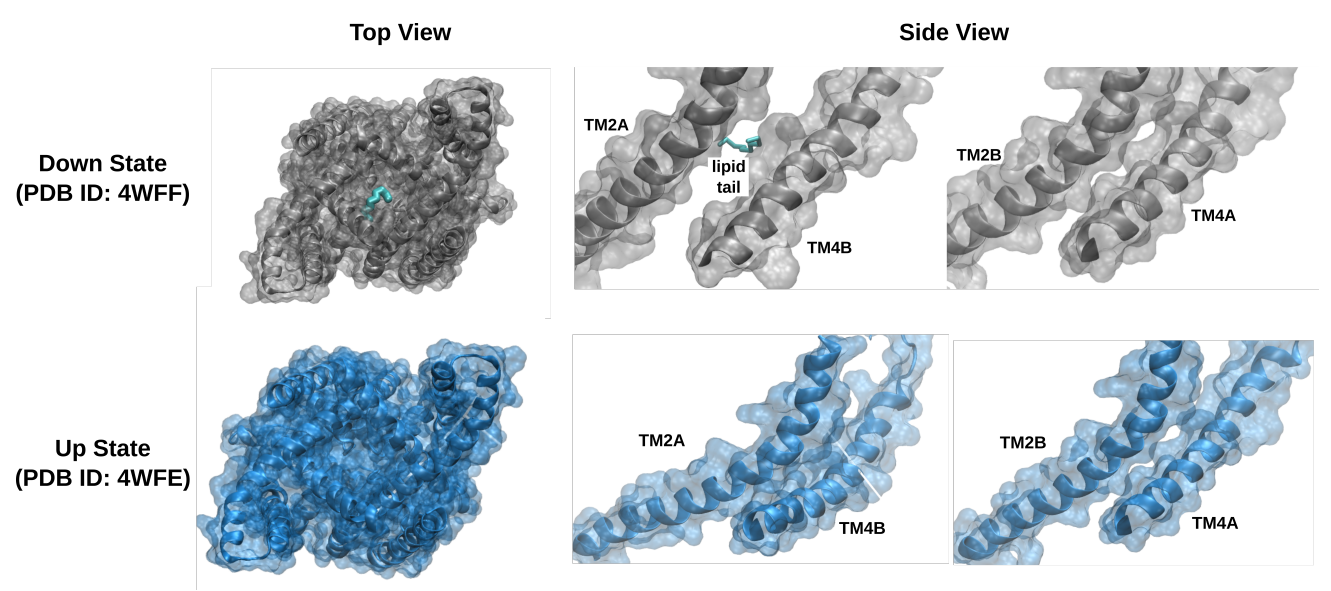

Figure 4.1.: Crystal Structures of the TRAAK Channel The crystal structures show two different states of TRAAK. The down state (PDB ID: 4WFF) shows a co-crystallized lipid tail (cyan) inside the channel. The TM4 helix of one subunit (TM4A) is in an up conformation whereas the other (TM4B) is in a down conformation. The down state opens a fenestration allowing a lipid to enter the inner cavity of the channel. The up state (PDB ID: 4WFE) has both TM4 helices in the up conformation. The closed fenestration excludes lipids from entering the inner cavity.

suggests, the TM4 helix of TRAAK is moved downward. This repositioning opens the fenestration for the lipid to enter. It was hypothesized that the lipid would block the ion entrance to the selectivity filter. A similar ion density was later found in a TREK-2 crystal structure, making a lipid block hypothesis more likely for K2P channels [17].

To further investigate the role of the lipid as blocking agent, two tests were performed by the same group who originally proposed the lipid gating theory [16]. The first test, the previously used unbranched lipids were replaced by branched diphytanoyl (DPhPC) lipids. Experiments using DPhPC lipids showed increased activity of the TRAAK channel supporting the lipid gating mechanism. Brohawn et al. speculated the branched lipids are unable to enter the cavity unlike the unbranched lipids, thus they are unable to block the cavity.

The second test was a modification of the TM2 and TM4 helices [16]: To create a disulfide bridge connecting the TM2 and TM4 helices, spatially close residues on these helices were replaced by Cysteines (I159C, R284C). At exposure to oxidizing solution, the disuplhide bridge is formed closing the fenestration and thus preventing lipid entrance. At exposure to oxidizing solution, the channel showed a more than 20 fold increased activation rate.

Taken together, these results lead us to investigate this potential mechanism of 
gating in more detail and with a theoretical approach. As molecular dynamics simulations allow a direct insight into the atomistic motions, they might allow direct insights into the mechanism as well. Therefore, we applied pulling simulations on the lipid partially entering the cavity in free simulations to achieve a lipid configuration similar to the one reported by Brohawn et al. Furthermore we performed the described double Cysteine replacement as well, introducing the disulfide bridge to study its effect on channel conductance.

The difference between simulations of the up and down crystal structures are central aspects of the studies presented in chapters 5 and 6 . In this chapter, we focus on simulations starting from the up configuration and the lipid block gating mechanism.

\subsection{Methods}

All simulations reported in this chapter were performed using the MD simulation package GROMACS 4.6 [75]. We try to resemble a physiological system with our setup. The force field used was AMBER99sb [76] with the SPC/E water model [77]. For ions Joung and Cheatham parameters were used [78] and the ion concentration was set to $0.6 \mathrm{M} \mathrm{KCl}$. The lipids were chosen to be Berger lipids 7981 . To treat the electrostatics, short-range interactions were cut off at $1.0 \mathrm{~nm}$ and longrange interactions were treated using PME 82, 83. To keep the temperature constant at $300 \mathrm{~K}$, the velocity rescaling thermostat was used [84]. The semiisotropic Berendsen barostat [85] was used to keep the pressure in z-direction at 1 bar and the surface tension in xy-direction constant at values ranging from $100 \mathrm{mN} / \mathrm{m}$ to $+100 \mathrm{mN} / \mathrm{m}$. Bonds were constrained with the LINCS algorithm [86]. Virtual sites were used for hydrogen atoms to allow for a 4 fs integration timestep [87, 88]. In simulations the Computational Electrophysiology setup was applied to keep a constant ion difference of $2 \mathrm{~K}^{+}$ions, resulting on average in a $400 \mathrm{mV}$ transmembrane voltage (refer to chapter 3) 68. For this setup two TRAAK channels, with initial coordinates from the up state PDB structure (PDB ID: 4I9W), was embedded in two membranes in a simulation box creating two compartments.

Initial simulations showed a lipid partially entering the cavity. This lipid was pulled into the cavity to resemble the configuration found by X-ray crystallographic studies using essential dynamics simulations 89 . One tail of the lipid reaching into the cavity was pulled with a constant force of $700 \mathrm{~kJ} /(\mathrm{mol} \mathrm{nm})$ along a vector. To keep the direction of the vector pointing towards the center of the cavity, position restraints on the heavy atoms of the protein were used. Subsequently, simulations were started from this configuration keeping the distance between neighboring 
atoms of the lipid and the fenestration fixed using umbrella distance potentials with a force constant of $6,000 \mathrm{~kJ} /\left(\mathrm{mol} \mathrm{nm}^{2}\right)$.

\subsection{Results}

For all simulations using the computational electrophysiology setup we observe continuous ion permeation when starting from the up crystal structure irrespective of the applied surface tension (see figure 4.2). This insensitivity to surface tension is in contrast to the experimental results which showed increased channel conductance as a result of applied surface tension. In contrast to the results from simulations of the up crystal structure, simulations starting from the down crystal structure do not show any permeation. Therefore, we exclusively focus on simulations of the up state.

As no surface tension dependence of the conductance is observed, the focus moved to studying different mechanisms of gating suggested in the literature. In free simulations, we found a lipid partially entering a fenestration, created by the TM2/TM4 helices, resembling a first step towards the location of a lipid identified in a crystallographic structure of Brohawn et al. (see figure 4.4) As the cavity of TRAAK is located at its center, a lipid able to block the ion permeations needs to enter the channel deeply through the fenestration. Such a deep penetration was not observed in our simulations. Lacking simulation time could explain the lipid's inability to fully enter the cavity and equilibrate. To overcome possible barriers along the entrance pathway and to test if the lipid would in principle be able to block ion permeation, the lipid was pulled into the cavity. The resulting structure shows the lipid tail occupying the cavity similar to the crystallographic structure (see figure 4.4).

Simulations of the resulting state without the applied pulling force, but with an additional force to keep the lipid inside the cavity (see Methods section), show the lipid retracts from the cavity in less than $10 \mathrm{~ns}$. In most of these simulations the lipid leaves the cavity completely irrespective of the distance restraints applied to it (see figure 4.4. In other simulations, the lipid moved close to the cavity wall. Both configurations allow the passage of ions (see figure 4.2 4.3. black line) and water into the cavity. Comparing the permeation rates of free simulations with simulations started from the lipid pulled in configuration shows no significant difference in permeability.

As permeation rates were not reduced by a lipid in the cavity, further investigations focused on introduced Cysteine residues. The replacement of two spatially close residues with a Cysteine (I159C, R284C) creating a disulfide bond increased con- 


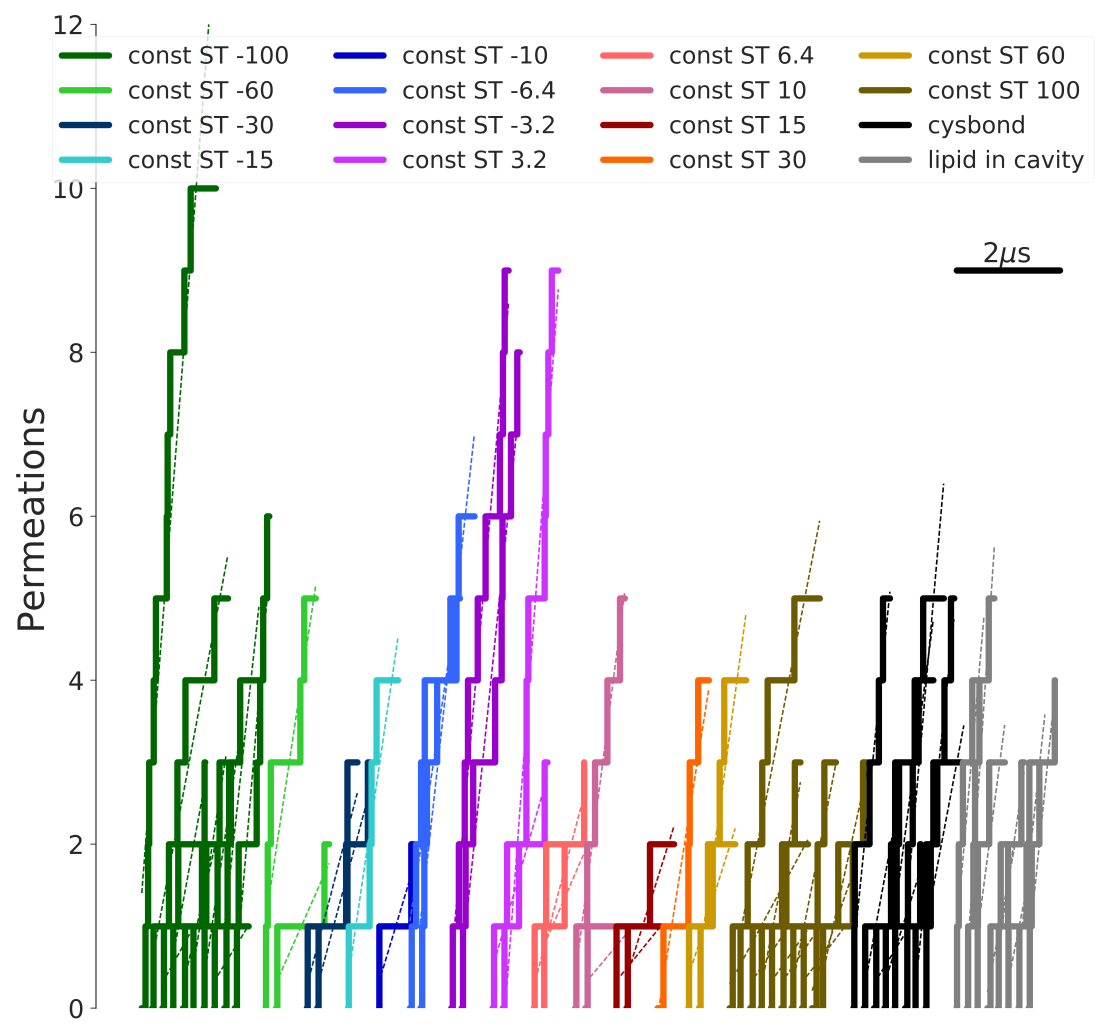

Figure 4.2.: Permeations of TRAAK Depending on Surface Tension Permeations are shown for simulations of TRAAK. Each step up represents the permeation of one potassium ion. The simulations vary in constant surface tension (const ST $[\mathrm{mN} / \mathrm{m}]$ ) they are sampled at, as indicated by the color. Additionally, simulations with a Cysteine bond (cysbond, black) and with a lipid in the cavity (gray) are shown. The time scale is represented as a black bar in the upper right. 


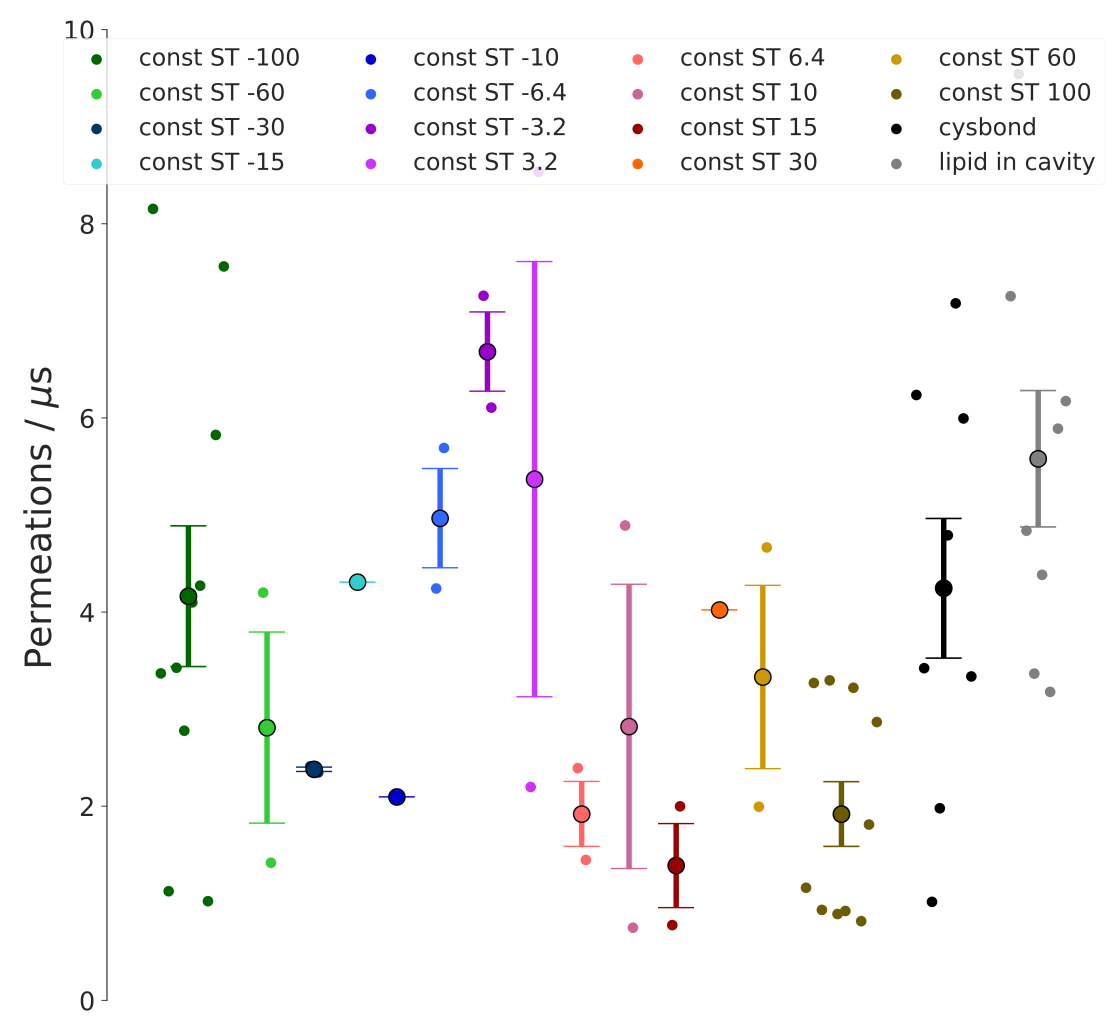

Figure 4.3.: Permeation rates of TRAAK Depending on Surface Tension Permeations of TRAAK are translated to permeation rates. The simulations are identical to 4.2. The dots represent the permeation rates of individual simulations whereas the filled circles with the errorbars represent the mean and the error of the simulations. 


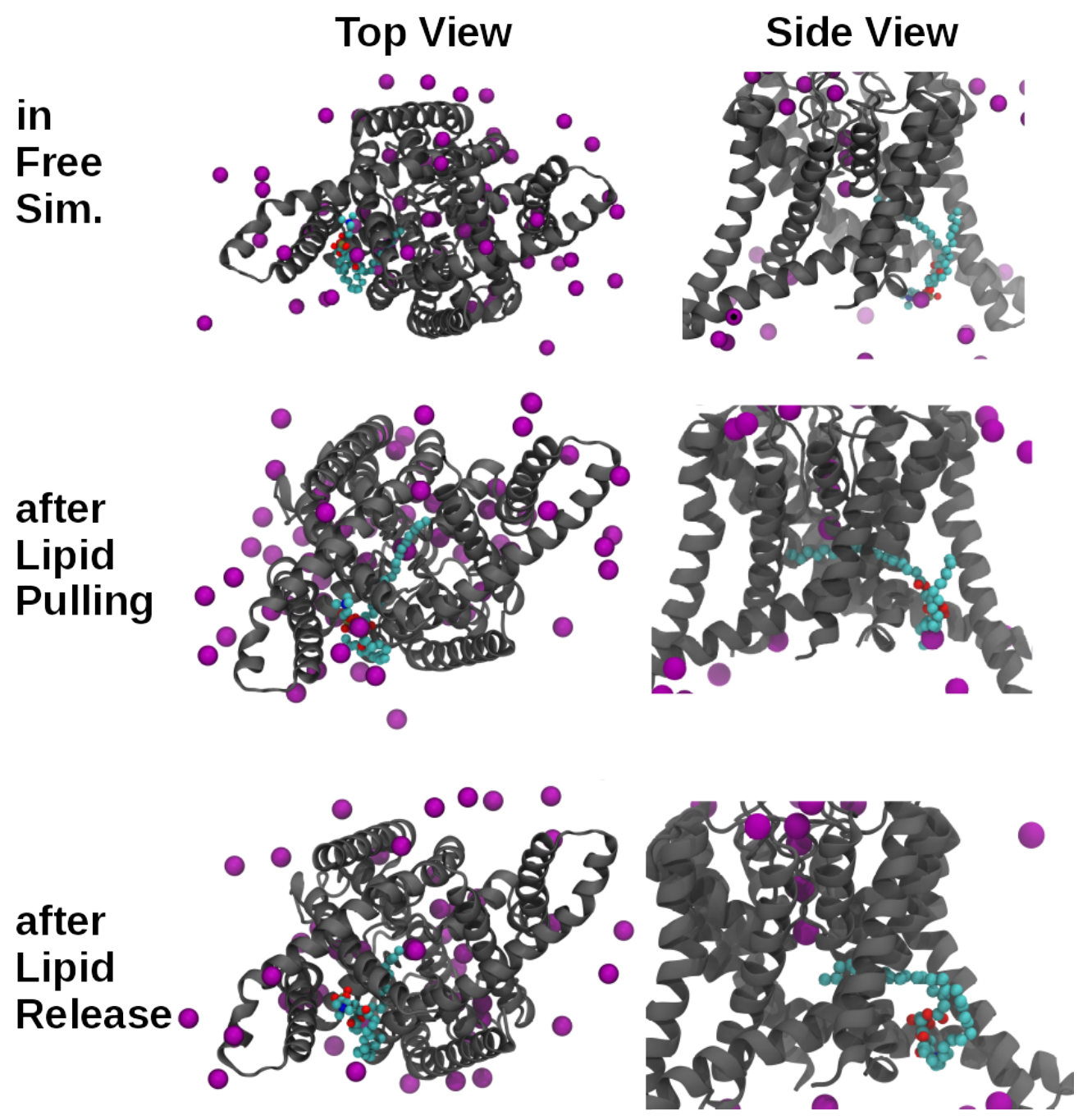

Figure 4.4.: Testing the Putative Lipid Block Mechanism The TRAAK channel is depicted as gray ribbons surrounded by potassium ions (purple spheres) and the lipid entering the fenestration (cyan and red spheres). Free simulations show a lipid entering the fenestration. The lipid does not enter as far as expected from the crystal structure (see figure 4.1). After pulling the lipid deeper into the cavity, its configuration is similar to the crystallographic down state (PDBID: $4 \mathrm{WFF}$ ). Release of the pulling force on the lipid results in a partial withdrawal of the lipid from the cavity, creating sufficient space for water molecules (not shown) and ions to enter the cavity. 
ductance experimentally. We tested this finding by introducing the two residues in both subunits of TRAAK and creating the disulfide bond. A fitting structure, with the mutated residues close together as the disulfide bonds require a certain distance, was selected as a starting structure for the mutations. Structurally the movement of TM4 is restricted in the simulations due to the disulfide bond. However, the permeation rates of these simulations do not show any significant difference 4.24.3, gray line) to simulations without the disulfide bond.

\subsection{Discussion \& Conclusions}

In none of the performed simulations did a lipid enter deeply enough into the channel cavity to block the ion permeation pathway. In addition, in simulations that started from a configuration with a lipid placed inside the cavity, the lipid left the cavity spontaneously. We conclude the cavity to be an unfavorable environment for the lipid. The tail of the lipid is hydrophobic whereas the cavity is often filled with water molecules. These results do not support the lipid block theory. An improbability of a lipid gate was later also found in another study [90]. However, these results were not available at the beginning of this thesis but support our own findings.

Furthermore, we tested the effect of introducing a Cysteine residue (I159C, R284C) showing an increased current in experiments [16] by MD simulations. Unlike the experimental results, the simulations of this double mutant did not show any effect on the conductance of the channel. We attribute this discrepancy to a lack of transitions between the conductive and non conductive state of the channel, a topic that is later explored in much more detail (see chapter 6). Without the change in the state, it is unlikely to observe any effect of the Cysteine mutation and the resulting disulfide bond. The explanation for the effect of the disulfide bond in the experiment was the occlusion of the lipid entry pathway. As we already discussed, we consider this gating mechanism unlikely. However, the difference in conductance of the channel with and without the disulfide bond could also be interpreted differently. As the bond restricts the TM4 helix to the up configuration, the stability of this configuration compared to the down configuration is increased. From the crystal structures a difference in conductance between these two states can be assumed. Thus, we suggest a selectivity filter based gating mechanism distinct from the lipid occlusion to be the alternative gating mechanism of $\mathrm{K} 2 \mathrm{P}$ channels. This alternative mechanism would not contradict the effect of the disulfide bond.

So far, no effect of the surface tension on the conductance was found. The next chapter looks into the probable gating mechanism. As we found lipid gating to be 
unlikely, a selectivity filter gate becomes more likely. In the next chapter we show how the selectivity filter gates and study the effect of membrane tension on the structural level as well as on the conductance. 



\section{Chapter 5}

\section{Mechanosensitive Gating of TREK-2}

This chapter has been published as Julian T. Brennecke and Bert L. de Groot. Mechanism of Mechanosensitive Gating of the TREK-2 Potassium Channel. Biophysical Journal (2018)

The mechanism of mechanosensitive gating of ion channels underlies many physiological processes including sensation of touch, hearing and pain perception. TREK2 is the best studied mechanosensitive member of the two-pore domain (K2P) potassium channel family. Apart from pressure sensing, it responds to a diverse range of stimuli. Two states, termed 'up' and 'down', are known from x-ray structural crystallographic studies that have been suggested to differ in conductance. However, the structural details of the gating behavior are largely unknown. In the presented work, we used molecular dynamics simulations to study the conductance of the states as well as the effect of mechanical membrane stretch on the channel. We find that the down state is less conductive than the up state. The introduction of membrane stretch in the simulations shifts the state of the channel towards an up configuration, independent of the starting configuration, and also increases its conductance. The correlation of the selectivity filter state and the conductance supports a model in which the selectivity filter gates by a carbonyl flip. This gate is stabilized by the pore helices. We suggest a modulation of these helices by an interface to the transmembrane (TM) helices. Membrane pressure changes the conformation of the TM helices directly and consequently also influences the channel conductance.

\subsection{Introduction}

In 1950, Katz first observed the translation of mechanical stress into depolarization of sensory nerve endings of frog muscles [91]. More than half a century later, 
we still do not fully understand the transition from mechanical stress to a change in conductance of ion channels. However, many important physiological processes, such as touch, hearing, balance and the sensation of pain, can nowadays be mapped to the function of mechanosensitive ion channels [35, 36].

Most of our structural understanding of mechanogating in ion channels comes from studies of the bacterial ion channels MscL and MscS 32. By computational studies, it was shown that in these channels, the surface tension of the lipid membrane directly induces a structural change $[92,93$. This mechanism was suggested before and is known as the force-from-lipid principle [47-49].

Despite the wealth of knowledge gained from these bacterial channels, it remains unclear to what extent the same principles hold for the structurally unrelated mammalian channels. The mechanogated mammalian ion channels TRAAK, TREK-1 and TREK-2 form the TRAAK/TREK subfamily of two-pore domain potassium (K2P) channels $[12$. Whereas MscL and MscS channels can gate by opening a direct ion pathway through the cell, K2P channels have a very stable region, the selectivity filter, which constricts the ions to a single file. Therefore, the mechanism of these channels is expected to be very different.

$\mathrm{K} 2 \mathrm{P}$ channels are present in a variety of cells and generate 'leak' or 'background' currents to stabilize resting membrane potential and represent important clinical targets for the treatment of cardiovascular diseases and several neurological disorders, including pain and depression [11].

Even before crystal structures of the members of the TRAAK/TREK K2P subfamily were available, studies showed that a gate in these channels had to be located in the selectivity filter and not, as in other ion channels, in the lower helix bundle [21 24]. More recent studies suggest a gating mechanism similar to C-type inactivation, where the gating takes place via a change in the selectivity filter conformation [27,74]. Crystal structures of the TRAAK and TREK-2 channel respectively revealed two different states, which were termed up and down 13, 17. The difference between these structures can only be found in the lower part of the channel we termed the lower helix bundle, but not in the selectivity filter (see figure 5.1). This lower helix bundle is more extended in the membrane plane in the up state while it points more into the cytoplasmic side in the down state. Ion occupancies of the selectivity filter suggested the up state to be conductive and the down state to be non conductive 13 15. In contrast, mutational studies on TRAAK reported the down state to be the stretch activated state, and by this conductive [20].

TREK-2 is the archetype channel of the TRAAK/TREK K2P subfamily on which 


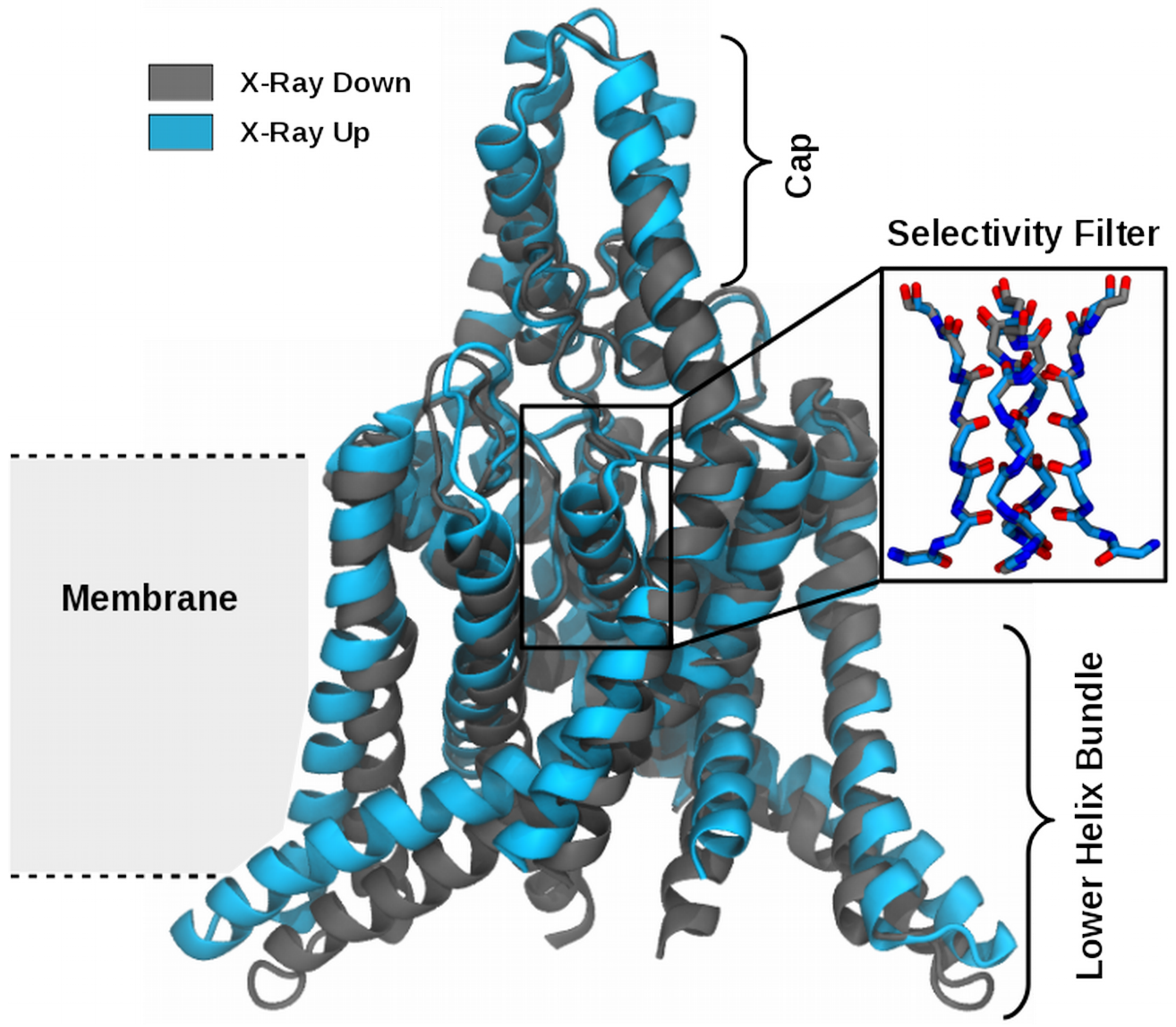

Figure 5.1.: Crystallographic Difference: Structural difference of the crystal up (4BW5, blue ribbon structure) and down (4XDJ, gray ribbon structure) structures of TREK-2 without C-terminus [17]. The major difference between the two configurations can be found in the lower helix bundle, whereas the configuration of the selectivity filter is indistinguishable (inset). On the top of the channel a cap region is located. The membrane the channel is embedded in is sketched in gray.

many of the mechanosensing studies are performed. It was extensively studied and has been crystallized in the up and down state, a prerequisite for accurate molecular dynamics (MD) simulations [17. It is not only known to play a role in thermosensation and pain perception 94,95 but is also an important pharmacological target 96. Multiple stimuli such as temperature, $\mathrm{pH}$, mechanical force, and several drugs modulate its activation [7-10]. These characteristics make TREK-2 a suitable system to study mechanogating in K2P channels.

Recent MD simulations on TREK-2 demonstrated the ability to switch the chan- 
nel from a down to an up configuration (and vice versa) by applying membrane tension (MT), whereas another non-mechanosensitive K2P channel did not show any response 90$]$. The simulations further suggested the ion occupancy in the selectivity filter to be altered by MT. In addition, extensive simulations with markov modeling revealed a pinched state of the selectivity filter which is destabilized by MT activation and is exclusively accessed in the down conformation [97].

Despite the insight gained from these studies, all results published so far were obtained without a physiological membrane potential and therefore do not yield insight into the conductance of the channel. We study the effect of membrane tension as well as the difference of the up and down state on the channel conductance. Therefore, we simulated TREK-2 in its up and down state with a membrane potential as well as with applied membrane tension.

\subsection{Methods}

The MD simulations with a total time of $28 \mu$ s were carried out using the GROMACS software package (version 5.0) [88, 98]. The up (PDB-Code: 4BW5) and down (PDB-Code: 4XDJ) crystal structures of the TREK-2 channel missing the Cterminus, which was demonstrated to retain stretch activation, [17] were embedded into a POPC lipid membrane, as common for mechanogating simulations 90,92 , and solvated in water with a $\mathrm{K}^{+}$ion concentration of $1.2 \mathrm{M}$ (as calculated by the number of ions relative to the number of waters in the system) and $\mathrm{Cl}^{-}$ions to neutralize the system.

\subsubsection{Amber}

MD simulations of membrane tensions were performed using the amber99sb forcefield [76 with SPC/E water [77], Joung et al. ion parameters [78] and Berger lipids [79 81]. Velocity rescaling [84 was applied for temperature coupling to a reference temperature of $300 \mathrm{~K}$. Berendsen pressure coupling [85] was applied to keep a constant pressure of 1 bar in the z direction with a time constant of $\tau=2.0$. For the $\mathrm{x}-\mathrm{y}$ direction (membrane plane), the constant surface-tension coupling 98 was used to keep it at the reference value. Initial velocities were sampled from a Maxwellian distribution at $300 \mathrm{~K}$. The bonds were constrained using the Lincs algorithm [86]. By replacing the hydrogen bonds with virtual sites 87, 88, internal vibrational degrees of freedom of the hydrogen atoms were removed, allowing for a 4 fs time step. Short ranged van der Waals interactions and electrostatic interactions were cut off at $1.0 \mathrm{~nm}$ each. The simulations were performed using periodic boundary conditions allowing to treat long range electrostatic interactions by the 
Particle Mesh Ewald summation 82,83] with a grid spacing of $0.135 \mathrm{~nm}$.

Missing atoms and loops in the crystal structure were modeled using the program loopy 99$]$. To integrate the channel into the membrane, the gmx membed program was used [100]. Subsequently, the system was minimized and equilibrated with restraints on the heavy atoms.

For each of the membrane tensions, four simulations of around $700 \mathrm{~ns}$ each were performed.

\subsubsection{Charmm}

Charmm (charmm36 ff [101]) simulations were set-up using the CHARMM-GUI [102 together with the membrane builder 102 104. The simulations were run using the GROMACS input generated by the CHARMM-GUI [105, 106].

The minimization and equilibration was performed using the standard GROMACS inputs of the CHARMM-GUI.

For conductance assessments, an external field of $400 \mathrm{mV}$ was applied [107]. This field creates a constant electrostatic potential on both sides of the membrane. The potential difference between the compartments thus equals the voltage of $400 \mathrm{mV}$. For the up and down state, 10 replicas of $600 \mathrm{~ns}$ each were run for each state, respectively.

\subsection{Results and Discussion}

\subsubsection{The TREK-2 Up State is more Conductive than the Down State}

Comparing the up (PDB-Code: 4BW5) and down (PDB-Code: 4XDJ) crystal structures, a difference can only be found in the lower helix bundle (Figure 5.1). These crystal structures suggest a conductive up state and a non conductive down state [17] while mutational studies suggest a conductive down state [20]. Not only the conductance of the different states is unclear, but also the mechanism by which the gating occurs.

To find a functional difference between the crystal structures, we simulated both states in a membrane with an external electric field of $400 \mathrm{mV}$ applied [107]. This setup gives us the opportunity to study the current through the channel while observing its motion in atomistic detail. We chose this setup as it more readily allows the application of a membrane pressure as compared to the double membrane setup utilized in computational electrophysiology [108]. 
Comparing the current of TREK-2 in simulations starting from the two differ-

Table 5.1.: Summary of Simulations Results

\begin{tabular}{lcc}
\hline & Up State & Down State \\
\hline Simulations with Relaxed Membrane & & \\
\hline Total simulation time $[\mu \mathrm{s}]$ & 7.4 & 7.3 \\
Nr. of ion permeations & 396 & 181 \\
Current [pA] & $8.6 \pm 1.8$ & $4.0 \pm 1.3$ \\
Current no flip [pA] & $13.6 \pm 1.6$ & $9.5 \pm 2.2$ \\
Nr. of water permeations & 19 & 19 \\
Flips [\%] & $37 \pm 12$ & $60 \pm 11$ \\
Permeations following direct knock-on [\%] & $90 \pm 7$ & $82 \pm 9$ \\
\hline Simulations with Stretched Membrane & & \\
\hline Current [pA] & $13.4 \pm 1.9$ & $12.9 \pm 1.5$ \\
Flips [\%] & $10 \pm 7$ & $10 \pm 10$
\end{tabular}

ent crystal structures, we found the channel in simulations starting from the up configuration to have a current of $8.6 \pm 1.8 \mathrm{pA}$, whereas simulations starting from the down configuration had a current of only $4.0 \pm 1.3 \mathrm{pA}$ (Table 5.1). From this difference we conclude that the up state is more conductive than the down state by a factor of two.

How this result compares to experiments is hard to address as in experiments the state of the channel cannot be determined while measuring a current. Likewise, the crystal structures do not allow us a direct readout of the difference in conductance. However, previous experimental studies on TREK-2 investigated single channel electrophysiology traces [8,109]. These studies consistently found multiple states of conductance, a closed state without any current and multiple open states which are a multiple of each other. The non conductance of the closed state is in agreement with our flipped state in which no ions permeate. Furthermore, the open state of the channel shows a current of $6 \mathrm{pA}$ at an applied voltage of $80 \mathrm{mV}$. These findings are in agreement with our simulations resulting in a current of $\approx 14 \mathrm{pA}$ at $400 \mathrm{mV}$ 
for the conductive state. Such an agreement is a strong indicator that we have sampled the correct mechanism of permeation. The additional open states could be due to multiple channels being measured simultaneously.

To find an explanation for the observed difference in conductance for the two states, we address the dynamic properties of the channel in the following.

\subsubsection{The Up State stabilizes the Selectivity Filter}

Previous studies suggest the ion gate to be located at the selectivity filter [21 24]. However, in the crystal structures, the selectivity filter of the up state is identical to the down state. As the crystal structures give a static picture only, we use the advantage of simulations to account for the flexible nature of proteins on short time scales and in atomistic detail. Therefore, we analyzed the behavior of the selectivity filter in relation to ion permeations.

In figure 5.2, the permeation events and the selectivity filter configuration of an example trajectory (up state) are shown. When the selectivity filter is in its crystallographic configuration, ions permeate at an even rate. In this example trajectory, a flip of one of the carbonyl groups (S3 in subfigure A) occurs after $150 \mathrm{~ns}$. At the same time, the ions stop permeating. At around $400 \mathrm{~ns}$ the flip is spontaneously reversed. We see that the ions start permeating again. When a flip occurs, we find a water molecule present in the selectivity filter sitting at the binding site of the flip as can be seen in figure 5.2 (A). In this configuration the flipped carbonyl and the water are stabilizing each other. The reversion of a flip to a permeating configuration is accompanied by the the water permeating and thus leaving the channel. The same behavior is consistent across all simulations and for up and down starting configurations. As we see events of water co-permeation, we further investigated the permeation mechanism. To analyze the mechanism we computed the number of permeations following the direct knock-on mechanism 15]. A permeation event is assigned to the direct knock-on type, if no permeating water can be found in the selectivity filter at the time of permeation. Both states clearly favor the pure direct knock-on mechanism, which more than $80 \%$ of the permeations exhibit (Table 5.1).

These results suggest the probability of flips to be the discriminating factor between simulations starting from the up and down structure. To test this hypothesis, we further analyzed the probability of flips in both states and also the current of the channels where no flip is present (Table 5.1). Indeed, we found that when only the non-flipped parts are taken into account, a more similar current is found for the up and down state. The difference which is not accounted for is mainly due to a single trajectory displaying a subconductive behavior. Consistently, we found that the probability of being in a flipped state is statistically significantly increased for 
A

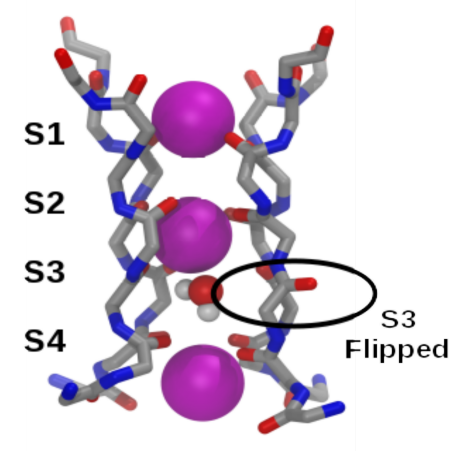

B

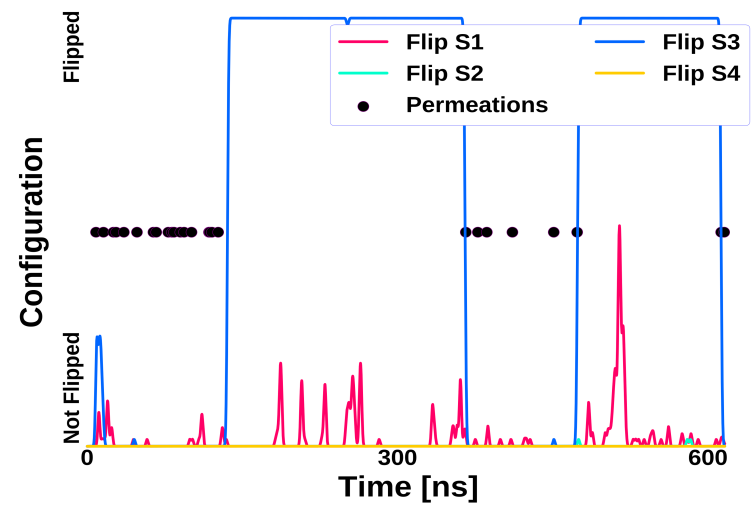

Figure 5.2.: Permeations and Carbonyl Flips: (A) The conformation of the selectivity filter is shown on the left. S1 to S4 correspond to the carbonyls separating the binding sites rather than to the binding sites themselves. The selectivity filter is filled with potassium ions (purple spheres). The carbonyl S3 shows a flipped configuration with a water molecule inside the selectivity filter. (B) Purple dots represent ion permeation events. In relation to this, the selectivity filter configuration is shown as lines (red corresponds to the state of the S1 carbonyl group, green - S2, blue - S3 and yellow corresponds to S4). If the line is at the bottom it represents a configuration where all carbonyls of the corresponding site of the selectivity filter are in their canonical configuration (as seen for example in figure (A) for S2). The top represents a state in which at least one carbonyl of the respective site is steadily flipped (as seen in figure (A) for S3). If the line is in the intermediate area it represents a temporarily flipped state (less than $1 \mathrm{~ns}$ ) and the height shows its duration.

If no flip is present the channel shows permeation events. When S3 flips after around $150 \mathrm{~ns}$, permeations stop. At around $400 \mathrm{~ns}$, the S3 carbonyl flips back into the canonical configuration and permeations resume. This behavior is representative for all simulations.

the down state, as expected. As the water block occurs together with the flip, a possible explanation for the difference in flips would be the probability of a water molecule to enter the selectivity filter, in contrast to a similar probability of a water entering the selectivity filter, but a different probability for a flip to occur when a water entered. In all 10 simulations of the up state, 19 water permeations occur (Table 5.1). This number is the same as for the simulations of the down state. The same number of water permeations in both states suggests the same probability of waters to enter the channel. Consequently, the probability for the carbonyls to flip depends on the state rather than on the water permeation probability. 
However, comparison between experiments and our simulations reveal a difference in timescales on which the channel switches between the two states. In our simulations the switching occurs on a ns- $\mu$ s timescale, whereas in experiments the gating occurs on a ms timescale. The comparison to experimental results makes us believe that the charmm forcefield, as applied in these simulations, samples the protein dynamics faster, leading to a reduced time required for selectivity filter state transitions.

In contrast to our findings, of a flip dominantly occurring in S3 (see figure 5.2 A), Aryal et al. found a flip of the selectivity filter occurring in the S0/S1 binding site when doing comparable simulations [90]. However, these simulations were performed without an external membrane potential. We found that the behavior of the ions in the selectivity filter changes significantly depending on the electric field. A spontaneous leaving of an ion at S0 is very likely with and without an external field. When an external field is applied, ions from the other binding sites tend to move in the void an ion leaving S0 leaves, preventing the carbonyls of that binding site to flip.

The probability of flips can explain the difference in conductance between the states. However, how the flip probability is altered by the overall configuration of the channel could not be resolved. TREK activator studies found that the selectivity filter can directly be activated by a ligand binding behind it. This study further suggests a mobility reduction of the pore helix - TM4 interface to be the reason for the activation [110]. Furthermore, structural analysis of functionally relevant mutations as well as differing wild type structures also suggest an allosteric transition of the information from the TM2/TM4 helices towards the selectivity filter [20]. Together with varies studies suggesting a selectivity filter gate, we find our results strongly supported $21,24,27,74]$. Based on these previous results and our own findings we suggest relative stability of the flipped versus non flipped configuration of the selectivity filter to be altered by the state of the channel and to therefore be the discriminating factor between the conductive versus non conductive state. Possible reasons for the flipped state of the selectivity filter not being observed in crystallographic studies so far could be due to its transient occurrence as well as due to a high ionic concentration used for crystallization, which likely stabilizes the canonical configuration. 

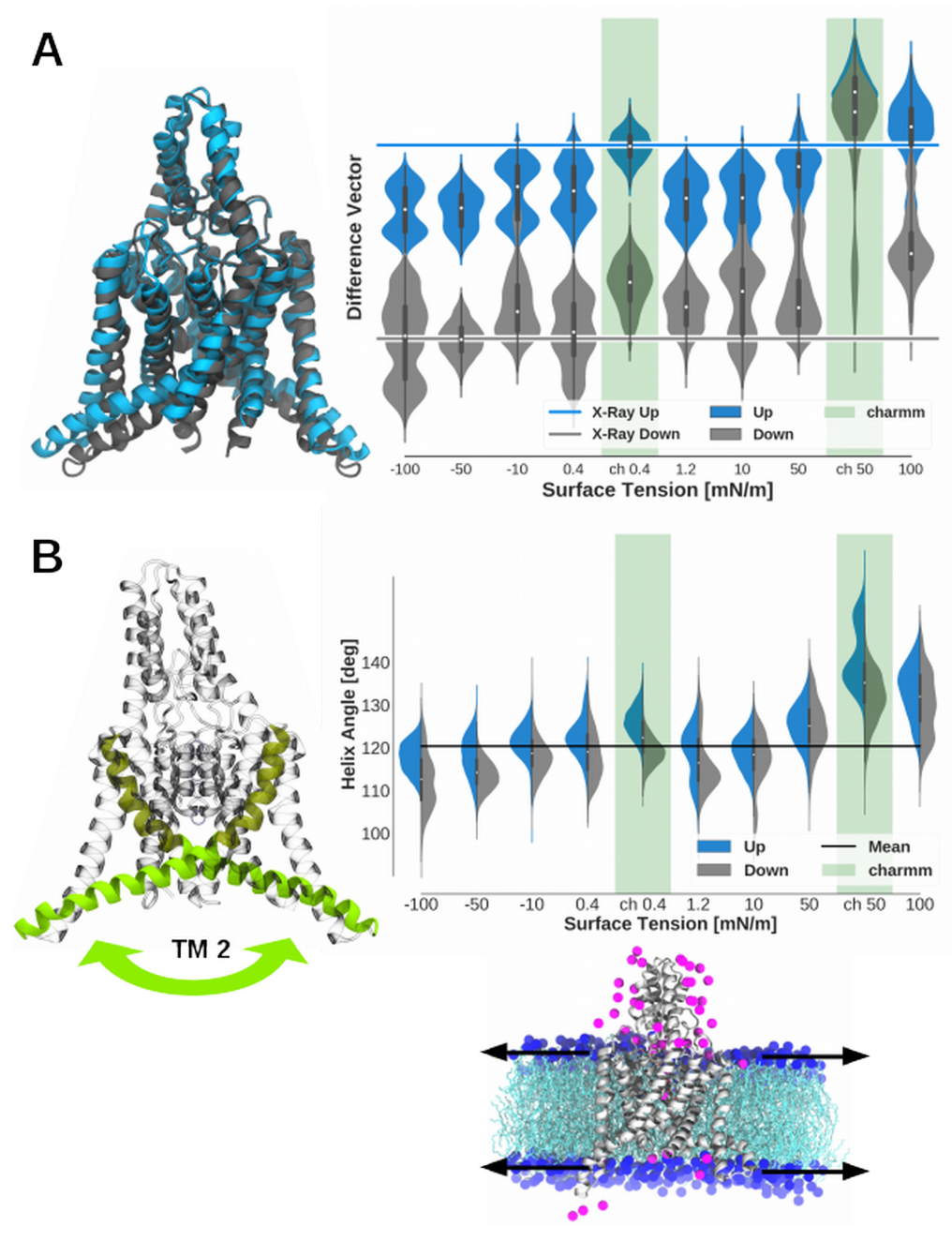

Figure 5.3.: Effects of Membrane Tension on the Up/Down Difference Vector and on the TM2 Helix Angle: (A) Projection of simulations at different membrane tensions projected onto the difference vector of the crystallographic states (excluding the cap). On the $\mathrm{x}$-axis the membrane tension is shown. The figure at the bottom represents the simulation setup where the channel is located inside the membrane and shown with some ions surrounding them. The black arrows show the stretch applied to the membrane. The gray line represents the configuration of the crystallographic down state (4XDJ), whereas the blue line represents the crystallographic up state (4BW5). Blue and gray violins represent the probability of the simulations starting from the up and down structures to be at the position on the difference vector. The dot in the violin represents the average over all simulations and the gray bars represent the variance of the data points summarized in the respective violins. MD simulations using the charmm force field are highlighted with a green background.

(B) To quantify the effect on the lower helix bundle we calculated the helix angle between the transmembrane helix 2 (TM2) of the two subunits. The violinplot shows the distribution of the helix angle for the different membrane tensions. 


\subsubsection{Membrane Stretch switches between Up and Down State}

So far, we identified a possible mechanism by which the conductance of TREK-2 can be linked to the different crystallographic states. To identify the mechanism by which the channel reacts on pressure, we performed further simulations. The majority of these simulations were performed in the amber99sb forcefield [76] and only two membrane tensions were simulated in the standard charm36 forcefield 101,102 as a reference.

When the pressure activation of the channel is measured in experiment, a suction is applied to the membrane, which leads to a distortion of the membrane, bending and stretching it simultaneously. The pressure activation of the channel is independent of the direction in which the pressure is applied, indicating a stretch activation rather than a membrane bend activation [12]. Results from Aryal et al. 90 suggest stretch activation of TREK-2 to occur by a down to up transition. To test this more extensively, we simulated TREK-2 at different membrane tensions and analyzed the effect of the stretch on the current and on the structure of the channel.

The current of the channel increases by membrane tension for both states and simulations starting from both states reach the same conductance of around $13 \mathrm{pA}$ (Table 5.1). By comparing the percentage of flips, we find a significant decrease (fisher test of the number of simulations with and w/o flips results in $p=0.001$ ). This decrease is likely the reason for the change in conductance.

To investigate if the pressure induced structural changes are related to the structural difference between the up to the down state, we used the crystallographic difference vector of TREK-2, without the cap region, and mapped the structure of the different membrane tension simulations after every 1 ns onto it (Figure 5.3 A). This transition mode incorporates the relevant motion of the protein.

The projection shows a significant movement towards the up state when the membrane is stretched. In contrast, the trend is towards the down state with no stretch or a compressed membrane. However, the simulations seem to be not fully converged yet as they do not show a gaussian distribution as expected. Focusing on the charmm trajectories, we see a full transition towards the up state. This full transition is compatible with the previously observed faster dynamics of the selectivity filter.

In the simulations with applied membrane pressure, we see a major effect on the transmembrane helix two (TM2). Therefore, we analyzed the reaction of these helices on the membrane tension in more detail. To do so, we used the same sim- 
ulations as previously mapped on the crystallographic difference vector. In these simulations, we calculated the angle between the TM2 helices of the two subunits. The results are shown in figure $5.3 \mathrm{~B}$. When a membrane stretch is applied, the angle between the TM2 helices is increased. This means the helices move further into the plane of the membrane. The compression of the membrane, in contrast, reduces the angle between the TM2 helices.

As the membrane tension is translated mainly in a change in the TM2 helices, we suggest that in the absence of the C-terminus, which is not resolved in the crystal structures, TM2 is the major force sensing domain. Looking at the effect of the membrane tension, we see a decrease of membrane thickness by stretch. We suggest the change in membrane thickness to be a major pathway to change the helix angle. We speculate that the movement of the lower helices, directly in contact with the membrane, propagate to the pore helices, which in turn affect the region behind the selectivity filter. As this is the area the carbonyls rotate into upon flipping, we suggest these changes to affect the flip probability, and with that the channel conductance. Further investigation on this mechanism will be part of subsequent investigation.

We propose a model of gating which extends the model of McClenaghan et al. 111. This model includes our findings for the difference in conductance, determined from the up and down starting configurations, as well as the effect of membrane tension on the channel configuration and current (Figure 5.4). We suggest up and down states have a different probability to be in either the conductive or non conductive state. When the channel is in the up state, it is more likely to be in the conductive state, whereas the down state increases the probability of being in the non conductive state. The conductive state is characterized by a canonical selectivity filter configuration, the non conductive state has a carbonyl flip in the selectivity filter, resulting in the disruption of the current. The effect of the membrane tension is to change the configuration of the channel. Stretch of the membrane moves the channel from a more down to a more up like configuration. Together with the relation of the up/down state to conductance of the channel, this model is proposed to explain the observed mechanogating.

\subsection{Conclusions}

To study the mechanogating mechanism of TREK-2 in atomistic detail, we performed MD simulations of the channel in a lipid membrane with applied membrane tension. The simulations revealed a factor of two difference in conductance for simulations started from the up and the down state. Further investigation on the 


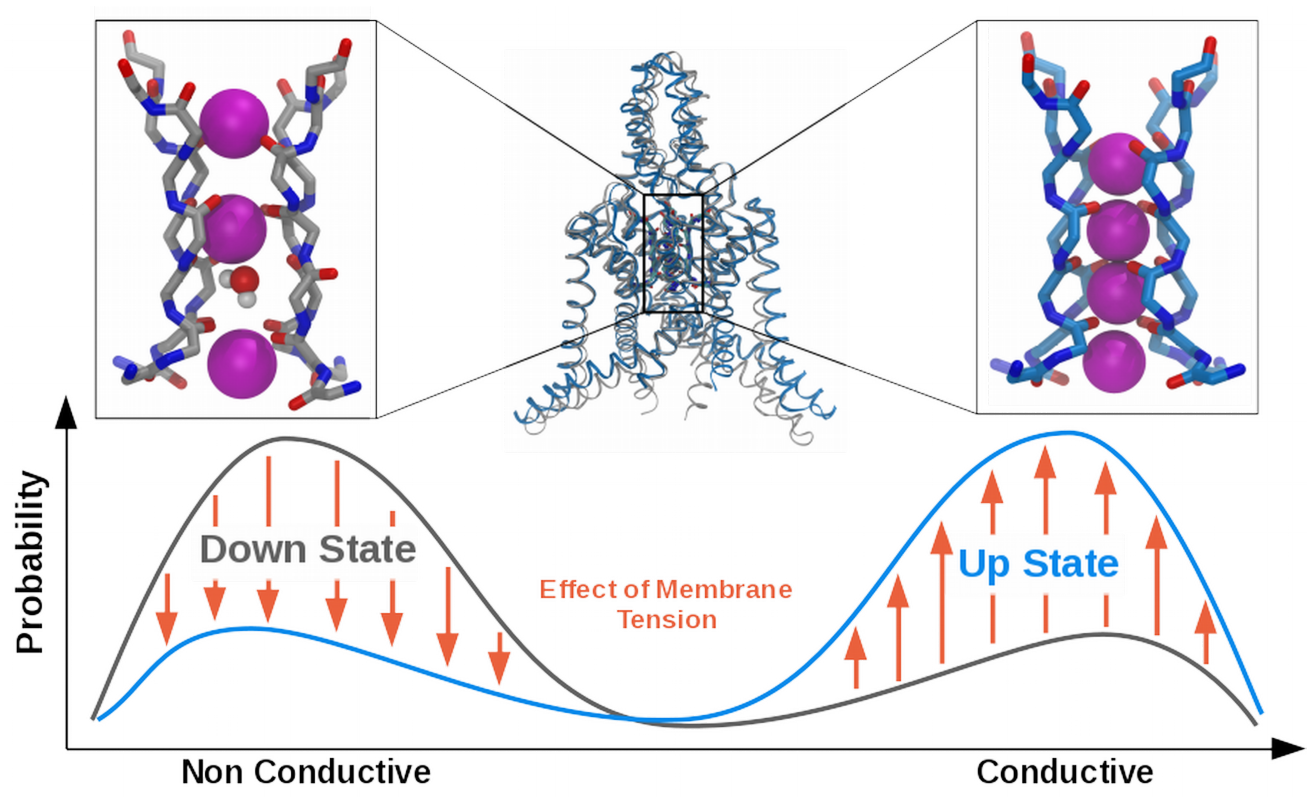

Figure 5.4.: Relation of Up and Down State to Conductance: Here, we summarize our results into a gating model. The down state (gray line) is more likely to be in a non conductive conformation than the up state (blue line). The down state is represented by a selectivity filter configuration with a flip and a water molecule in the SF. In contrast, the up state is more likely to be in a conductive configuration as represented by a canonical SF with full ion occupancy. Applying membrane tension (orange arrows) changes the state of the protein towards a more up like configuration which is more conductive.

underlying reason suggests a difference in the relative stability of the selectivity filter states to be the distinguishing factor for conductance. In the up state the selectivity filter is less likely to adopt a non conductive conformation compared to the down state. The non conductive conformation is characterized by a deviation from the canonical selectivity filter configuration, the flipped conformation, characterized by an outward pointing carbonyl group. This flipped conformation prevents ions from passing through the filter and is frequently accompanied by a water molecule in the filter.

The results are consistent with a proposed mechanism by which the transmembrane helices (TM) TM2 and TM4 change the interface of TM4 with the pore helices behind the selectivity filter $[110]$. We suggest that this change of the interface subsequently alters the relative stability of the selectivity filter states.

Consistent with findings reported by Arial et al., we found membrane tension to alter the state of the channel, and especially of the lower helix bundle, to adopt a more up or down like conformation by membrane stretch and compression, re- 
spectively [90]. This results in an increase of current by membrane stretch.

Based on these results, we propose the following model of gating which is illustrated in figure 5.4. In this model, applied membrane stretch makes the channel more likely to adopt the up state compared to the down state. Furthermore, the up state stabilizes the selectivity filter, leading to an (on average) more conductive channel as compared to the down state. This mechanical tension of the membrane can thus translate into a change in conductance of TREK-2.

\subsection{Author Contributions}

JB and BG developed the concept of the study. JB prepared and performed the simulations and analysis. JB and BG wrote the paper.

\subsection{Acknowldegments}

We thank Han Sun for providing the starting structures of the MD simulations. Funding by the Deutsche Forschungsgesellschaft (DFG) Sonderforschungsbereich (SFB) 803: Project A03 to JB and BG is gratefully acknowledged. 


\section{Chapter 6}

\section{Selectivity Filter Exchange}

\subsection{Introduction}

In the last chapter on mechanosensitive gating (chapter 5), we found the selectivity filter (SF) to be decisive for the conductance of TREK-2. The simulations revealed a conductive canonical configuration and a non conductive flipped configuration of the SF (see figure $5.2 \mathrm{~A}$ ). Both configurations are sampled by simulations of the two starting structures, termed 'up' and 'down', and the obvious correlation between SF configuration and conductance suggest a SF gate. Thus the configuration of the filter determines the conductance, rather than other structural features of the up or the down state.

To reduce the forcefield (FF) dependence of our results, we simulated the same system in a different FF. Here we present results obtained using Amber99sb and compare them to our previous results obtained using Charmm36. In these new simulations, only one SF configuration for each of the starting structures is sampled: Simulations started from the up state constantly adopt a canonical SF configuration, down simulations adopt a flipped SF configuration (see figure 6.1). Thus only a correlation between SF configuration and conductance can be assumed as structural feature distinguishing up and down and is the potential reason for the conductance difference.

To test whether the conductance originates from the SF configuration or from another undetermined factor, we exchanged the SF of the two structures creating an up state with a flipped SF and a down state with a canonical SF configuration. Simulations started from these structures allow us to disentangle the role of the overall protein state and the SF configuration.

The canonical SF configuration restores $1 / 3$ of the original conductance when inserted into the down state, whereas the flipped configuration prevents ion permeations in the up state. Together with the determined conductance of the free 


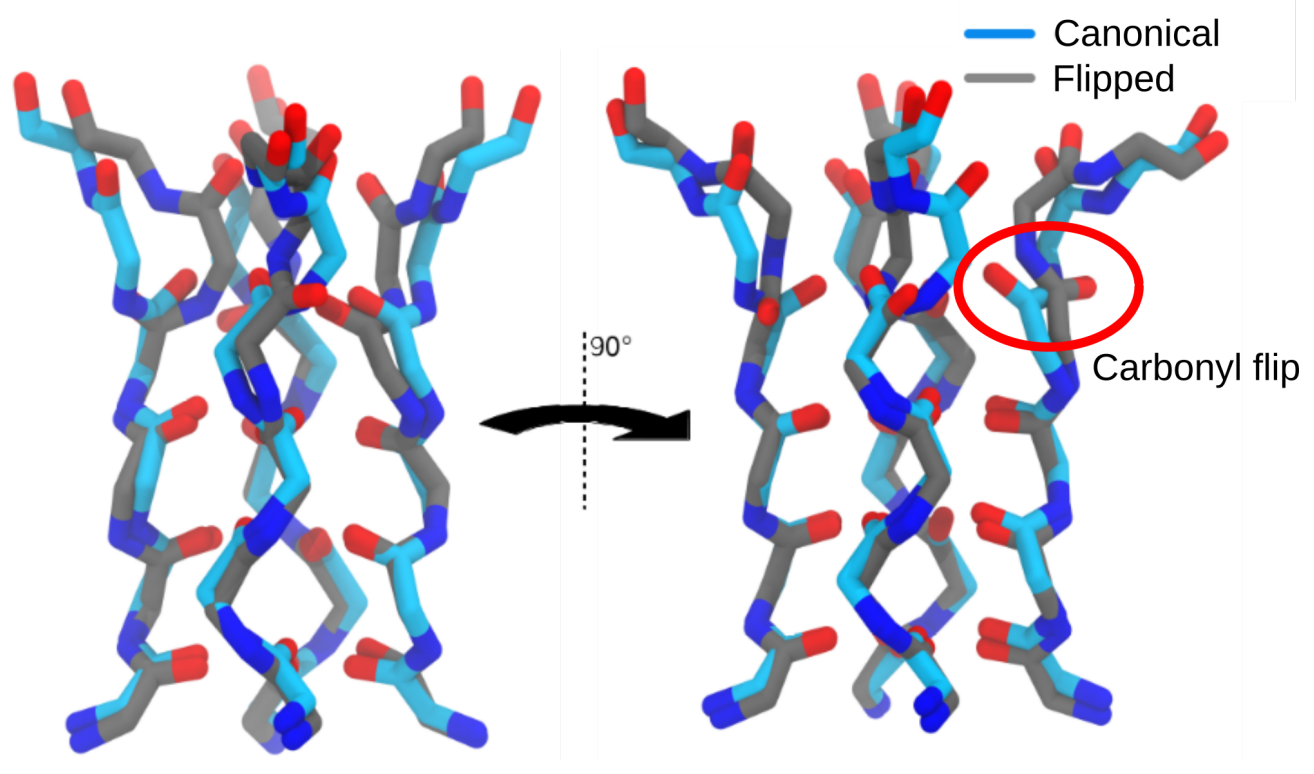

Figure 6.1.: Superposition of Canonical and Flipped SF The SF of the up state simulations (blue structure) shows a canonical configuration and allows ion permeation. In contrast, the $\mathrm{SF}$ of the down state simulations (gray structure) shows a carbonyl flip (red circle) after equilibration stopping ion permeation.

simulations, we conclude a SF gate beyond a simple correlation between the conductance and the filter configuration supporting our previous results.

\subsection{Methods}

The used simulation setup is identical to the setup used for simulations in the Amber99sb FF described in chapter 5 and the relevant results of the original simulations are reported there. In total, eight random frames were selected from these simulations, four simulations started from the up state (canonical) and four started from the down state (flipped) (figure 6.2). The SF of the protein in the selected frames was cut out and exchanged between a pair of up and down states. As the configuration of the SF differed between the pairs, they did not match the vacancy created by cutting out the original SF. Thus, an extensive energy minimization was required to fix inappropriate distances connecting the protein structure to the exchanged SF. We reduced the disturbance of the SF in the minimization by applying position restraints on the heavy atoms of the SF. For the minimization, the steepest descent algorithm was used. Subsequently, an equilibration simulation was performed for $30 \mathrm{~ns}$, using the same parameters as for the simulations with additional position restraints on the SF. To test the configuration of the SF, we 


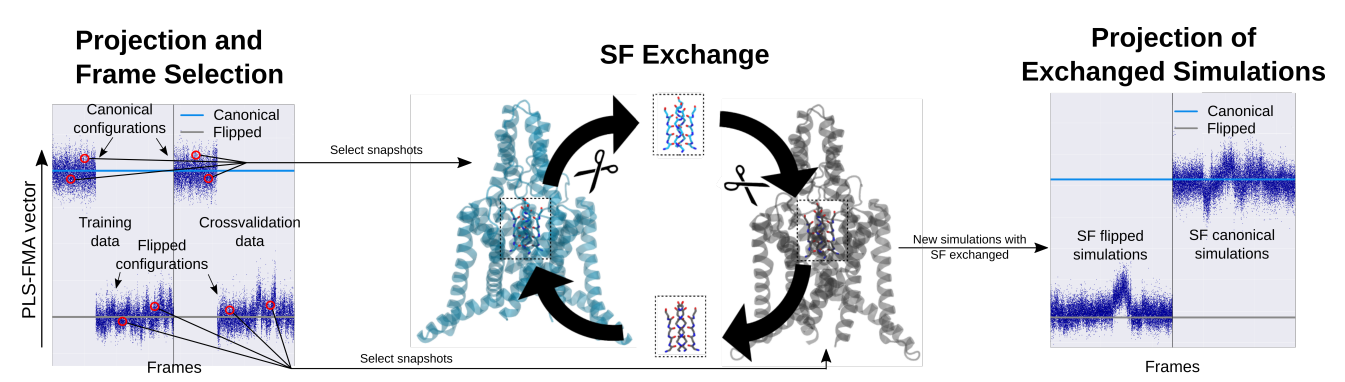

Figure 6.2.: SF Exchange Scheme Using PLS-FMA, we were able to distinguish between canonical and flipped configurations of the SF by a projection onto the PLS-FMA vector. To create the model, one half of each simulation data was used. The other half was subsequently used for cross validation. Four structures were randomly selected from canonical and flipped SF configuration simulations (red circles). To identify the effect of the SF on the conductance, we exchanged the SF of snapshots of the canonical configuration with snapshots of the flipped configuration. Simulations of these exchanged structures were started. The SF was projected on the same PLS-FMA vector to determine its stability after the exchange, as it appears to be.

\begin{tabular}{c|cc|cc} 
& \multicolumn{2}{|c|}{ Before Exchange } & \multicolumn{2}{c}{ After Exchange } \\
\hline SF config. & canonical & flipped & canonical & flipped \\
state & up & down & down & up
\end{tabular}

Table 6.1.: Overview of Simulated systems

applied PLS-FMA. Each frame of the free simulations was labeled to originate from a canonical or a flipped SF configuration, corresponding to an up or a down state. For training, half of the canonical and half of the flipped SF configurations were used. The other half was used for cross-validation.

\subsection{Results}

The PLS-FMA model of the SF distinguishes two states along the PLS-FMA vector (see figure 6.2). The state at the bottom represents the flipped SF configuration whereas the state at the top represents the canonical configuration. Simulations started from an exchanged SF (see Methods section) were projected onto the same vector to determine if the SF configuration remained stable after the exchange. SF flipped configurations are expected to remain at the bottom around the gray line, used as an indicator for the state, whereas canonical SF configurations are expected to remain at the top after the exchange. As no configuration change was 


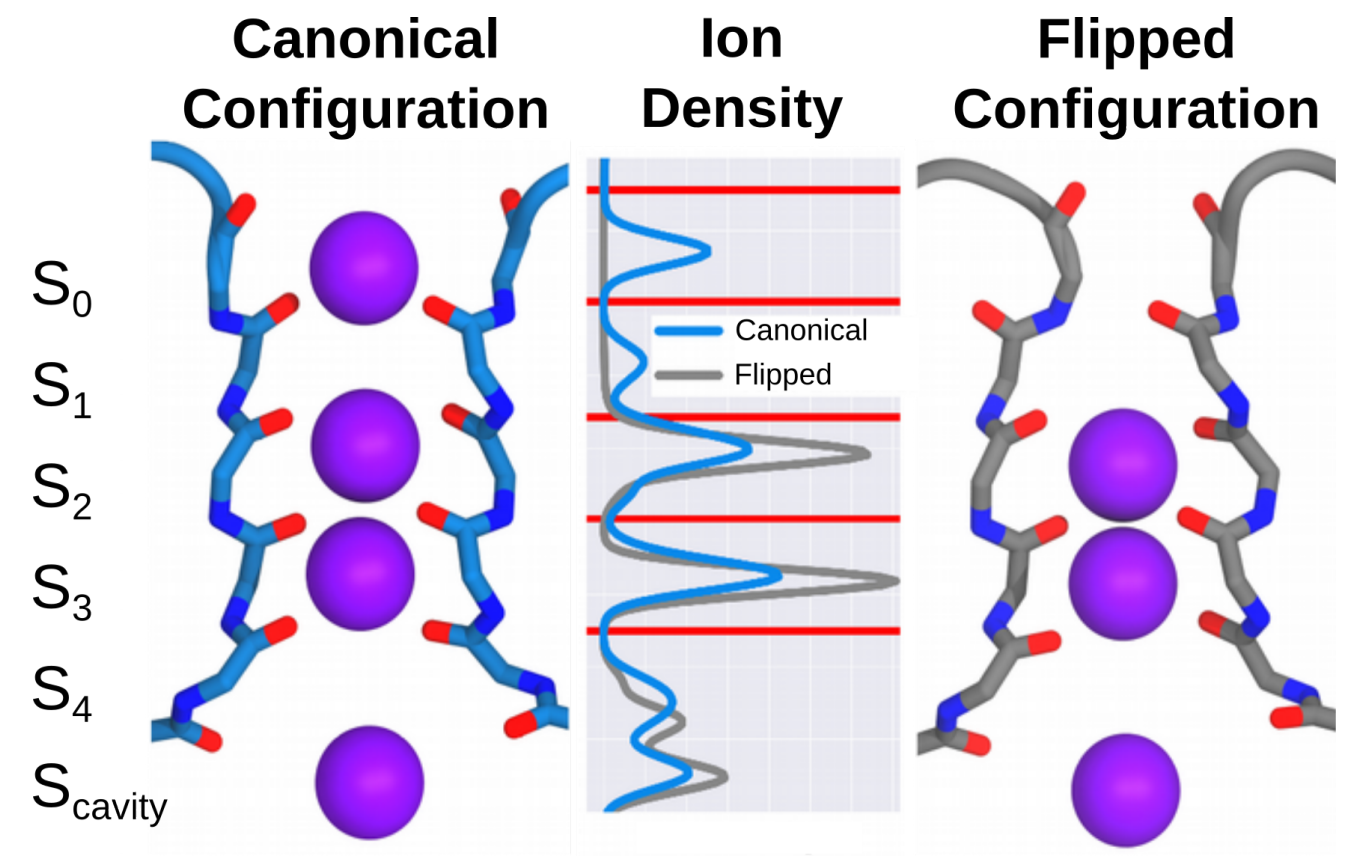

Figure 6.3.: SF Ion Density Distribution Canonical (blue) and flipped (gray) configurations of the SF show different ion density at the binding sites $\left(\mathrm{S}_{0}-\mathrm{S}_{4}\right.$ and $\mathrm{S}_{\text {cavity }}$ ). The configurations and the ions (purple spheres) shown are representative for the simulations. The flipped configuration, corresponding to the down state, shows reduced ion density of the SF. The red lines mark the position of the SF carbonyls. 
observed, we assume sufficiently long minimization and equilibration to create a stable configuration in which the SF remains in the intended state.

Permeation rate analysis after SF exchange reveals total discontinuation of permeation in the up state with a flipped SF. In contrast, the down state with the canonical SF starts to permeate potassium ions. The resulting current is $0.27 \mathrm{pA}$ at a voltage of $400 \mathrm{mV}$. This current is a reduction to $1 / 3$ of the original current for the up state with the canonical SF (original current $0.75 \mathrm{pA}$ ).

To identify the origin of the difference between the canonical SF permeations in the original simulations and after the exchange, we analyzed the effect of the SF on the potassium ions directly. In figure 6.3, the ion density of the $\mathrm{SF}$ is shown for the canonical (up state) and the flipped (down state) configuration. The ion density is defined as the probability to find a potassium ion at a given position inside the SF in any frame of the simulation. For the canonical SF configuration, we find ions occupying all five binding sites inside the SF (see figure 6.3, area between red lines) as well as the cavity. In contrast, no potassium ions occupy the upper most two binding sites $\left(\mathrm{S}_{0}, \mathrm{~S}_{1}\right)$ in the flipped $\mathrm{SF}$ configuration. The interruption of the permeation pathway by blocking ions in the upper most part of the SF likely determines the ability of the structure to permeate.

After SF exchange, the ion density of the flipped SF inserted in the up structure is similar to the ion density before the exchange (see figure 6.4). The missing density at binding sites $S_{0}$ and $S_{1}$ in the flipped $S F$ in the original and in the exchanged simulations is a potential explanation for the blocked ion transfer through the channel.

The canonical SF inserted into the down structure shows mostly a recovery of the ion density of the up simulations. Only minor deviations are visible right of the $S_{0}$ binding site and left of the cavity (gray background, figure 6.4). In both these areas, SF exchanged simulations show a reduced ion density compared to the original up simulations.

\subsection{Discussion \& Conclusions}

In the chapter on mechanosensitive gating (chapter 5), we found that the state of the SF is affected by the surrounding channel state, especially by the lower helix bundle. In the simulations presented in this chapter, we do not observe any transition between the flipped and the canonical configuration of the SF. Thus we exchanged the SF of structures from up and down state simulations to test the effect of the SF configuration independent of the surrounding channel state. Simulations primarily analyzed in the previous chapter and the simulations pre- 


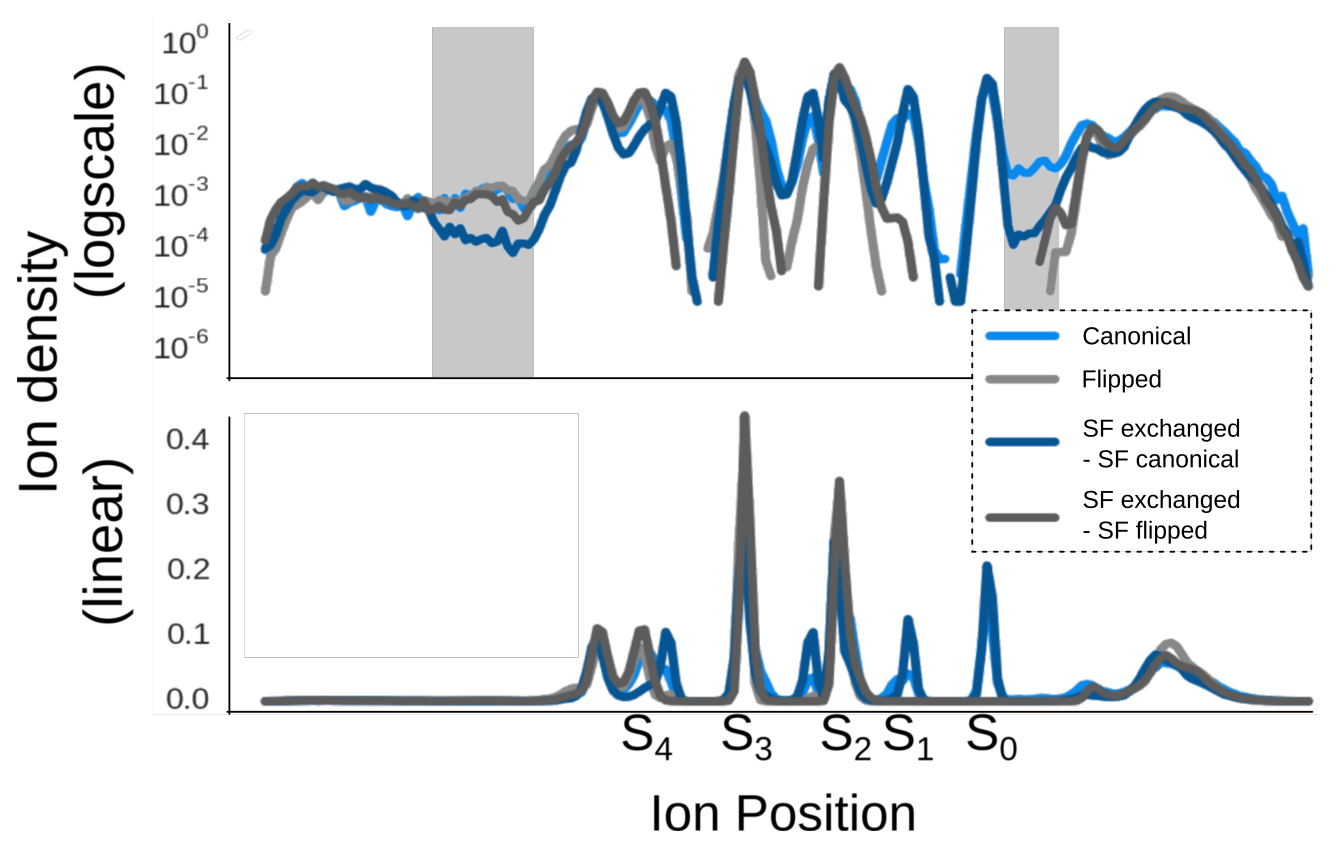

Figure 6.4.: SF Ion Density after the Exchange The ion density in and around the SF (binding sites $\mathrm{S}_{0}$ to $\mathrm{S}_{4}$ ) is shown at linear- and logscale for simulations with a canonical (light blue) and a flipped (light gray) SF configuration and for the SF exchanged simulations (darker color), respectively. After the SF exchange the ion density inside the SF is exchanged as well. Minor differences between simulations of the canonical SF and SF exchanged simulations of the canonical SF are highlighted (gray background). 
sented here differ in the respective $\mathrm{FF}$ to reduce the dependence of our results on the FF. Here we used Amber99sb, whereas results of the previous chapter were obtained using Charmm36. Our observation of a SF flip on a $100 \mathrm{~ns}$ time scale in Charmm36 simulations compared to a stable SF in the Amber99sb simulations during the entire simulation time $(\mu s)$ suggests a difference in simulation time required to sample relevant conformations in these two FFs. Comparing gating time scales observed in simulations to experimental results, from which we expect gating on the $\mu$ s time scale, we find Charmm36 to overestimate the flexibility of the SF resulting in increased configuration transitions by a factor of ten, whereas Amber99sb underestimates its flexibility by an unknown factor. We believe the correct gating kinetics to be an intermediate between the two FFs. Simulations of both Amber99sb and Charmm36 show gating of the SF. Evidence of a SF gate in $\mathrm{K} 2 \mathrm{P}$ channels was previously found by experiments [21-24, 74]. Therefore, we assume that we determined the responsible conformational change representing $\mathrm{K} 2 \mathrm{P}$ channel gating.

We expect the ion density of the up state simulations with a canonical SF configuration to show a clear indication of five well defined ion binding sites $\left(\mathrm{S}_{0}-\mathrm{S}_{4}\right)$. These five ion binding sites are represented by a locally increased ion density. A sixth increased ion density represents the cavity of the channel. In the down state simulations with a flipped SF configuration, the ion density of the upper most two binding sites $\left(S_{0}, S_{1}\right)$ is reduced to zero. The reason for the loss in ion density is most likely the carbonyl flip, disrupting the permeation pathway of the ions by removing spatially close free energy minima. Consequently, the flip directly increases the barrier ions have to overcome to permeate the channel. A flipping mechanism causing loss of ion density would agree with the idea to discriminate conductive and non conductive states by the average ion density of the SF [14.15]. However, results of Schewe et al. 74 suggest a gating charge of $\approx 2.2 \mathrm{e}^{-}$for $\mathrm{K} 2 \mathrm{P}$ channels. From our simulations we obtain a gating charge of $\approx 1.0 \mathrm{e}^{-}$for the Charmm36 FF and $\approx 2 \mathrm{e}^{-}$for the Amber99sb FF. The higher gating charge observed in experiments, suggests more ions leaving the selectivity filter at gating.

In contrast to the results of the previous chapter where flips dominantly occurred at the carbonyl between $S_{2}$ and $S_{3}$, here we find the flips occurring dominantly at the upper most carbonyls $\left(S_{0} / S_{1}, S_{1} / S_{2}\right)$. An obvious explanation for the difference is again the effect of the used FF. However, there is an additional indirect difference. The flips of the simulations in the Amber99sb FF did already occur during the equilibration and persisted for the whole simulation time. This behavior differs greatly from simulations in the Charmm36 FF which showed flips and also recovery from flips during simulations. An important difference between the equilibration and the actual simulation is the applied transmembrane voltage. 
Thus flips of the upper most carbonyls did occur without a transmembrane potential whereas flips at $S_{2} / S_{3}$ did occur with the potential applied. Simulations using the Charmm36 FF without a transmembrane potential performed by Aryal et al. [90] did show a small ion density at $S_{0}$, around 5 times smaller than in our simulations using the same FF. Applying an additional membrane stretch to make the channel more conductive resulted in an increased ion density at this binding site suggesting a relation between conductance and ion density at $S_{0}$ [90. As simulations without a transmembrane potential show a change in ion density at the upper ion binding sites across FFs, whereas the same FF used by Aryal et al. shows a change in $S_{3}$ ion density, we suggest the transmembrane potential to be the discriminating factor for the location of the change in the SF. Ions located at $S_{0}$ can leave the SF. However, if a transmembrane voltage is applied, the ions of the lower binding sites will be more likely to replace this ion. If all ions move up, the lowest ion binding site becomes unoccupied.

After exchanging the SF of the up and the down state of the channel, conductance of the original simulations is not fully recovered. The flipped SF inside the up channel abolishes ion permeation completely suggesting the SF to be the important factor for non conductance. However, the canonical SF inside the down channel does only create $1 / 3$ of the current of the original up state simulations. The difference in conductance might have two explanations: Either an additional structural feature of the down state is responsible for the subconductance, or the SF configuration is not fully conserved after the exchange. Looking at the probability of ions entering the cavity and the water molecule distribution inside, which are similar for the up and down simulations, we do not suggest an additional structural feature of the down state to cause subconductance. By looking at the ion density after the exchange, some changes, especially right of $S_{0}$, could suggest slight differences in the SF between original and exchanged simulations. This idea is further supported by the projection of the SF conformation onto the canonical-flipped PLS-FMA vector. After exchanging the SF, projections of subsequent frames are more correlated with each other. This correlation hints at structural changes in the SF in degrees of freedom perpendicular to the PLS-FMA vector. A new set of simulations could be performed to reduce the disturbance of the SF, with a higher force constant for the restraints on the selectivity filter at minimization and equilibration and a longer equilibration time.

Furthermore, we simulated the down structure with full ion density resulting in a canonical SF configuration. These simulations showed identical permeation rates to up simulations, indeed suggesting a gate exclusively located in the filter.

As the flipped SF configuration is asymmetric, application of the (a)symmetry tools introduced in chapter 7 could be reasonable. However, as the flip occurs 
during the equilibration phase, it is similar between all trajectories.

Taken together, the results of the SF exchange in the Amber99sb FF support our findings in regard to the gating mechanism from simulations in the Charmm36 FF. Simulations of both FF suggest a SF gate which is likely influenced by the lower helix bundle rather than a gate outside of the SF. 



\section{Chapter 7}

\section{Quantifying Asymmetry of Multimeric Proteins}

This chapter is currently under review as Julian T. Brennecke, Bert L. de Groot. Quantifying Asymmetry of Multimeric Proteins. Journal of Chemical Physics

A large number of proteins assemble as homooligomers. These homooligomers accomplish their function either symmetrically or asymmetrically. If asymmetry is prevalent in a structure ensemble, the asymmetric motion will occur in any of the subunits. Many computational analysis tools implicitly use ensemble averages to determine protein motions, e.g. Principal Component Analysis. Therefore, taken together, this approach results in a loss of the asymmetric signal and a false symmetric output, rendering it impossible to analyze asymmetric motions with available tools.

A first step towards understanding asymmetric systems is the quantification of asymmetry. Only a few tools exist to calculate asymmetry quantitatively, such as the Continuous Symmetry Measure (CSM). In this study, we present an extension of CSM delivering additional information about the subunit contributions to the overall asymmetry. Furthermore, we introduce an algorithm termed Functional Asymmetry MEasure (FAME). FAME is based on the algorithm PLS-FMA and allows calculating asymmetry in relation to protein function.

To validate our developed algorithm, we applied it to two different potassium channels, TREK-2 and KcsA, as well as to the unfolding mechanism of the carrier protein Transthyretin. For both potassium channel systems an artificial asymmetric motion was introduced to benchmark the algorithm in addition to demonstrate the interpretation potential of the results. Therefore, the degree of overall as well as subunit based asymmetry for KcsA was quantified using CSM as the provided extension requires more than two subunits. The functional modes of asymmetric TREK-2 motions were recovered and their asymmetry was quantified using FAME 
as a dimeric protein is the simplest application. FAME was further used to study the asymmetry of the unfolding pathway of Transthyretin.

We show the ability of both algorithms to correctly predict asymmetry. The tools are available online and can be applied to most homooligomeric systems.

\subsection{Introduction}

A major fraction of all proteins is functionally active in a homooligomeric form and many of these are symmetric [112 115]. This dominance of symmetric structures can only be explained by an evolutionary advantage of symmetry [112]. Among others, increased stability of symmetric homooligomers [114, 116, 117], a well defined number of subunits in the multimer, [112] as well as favorable folding pathways of symmetric structures 118 are speculated to be the evolutionary driving force.

However, protein functions are known which indeed require structural asymmetry, such as unidirectional motion in polymerases and ribosomes [112, or reciprocal symmetry (the adoption of different states in the subunits) as in the ATP synthase [119]. Furthermore, according to the MWC model of allostery, symmetry is a prerequisite for allosteric proteins [120]. The MWC model even suggests that the conservation of symmetry causes allostery. While binding of a ligand to one subunit, a structural change in all subunits is initiated, further facilitating the binding of similar ligands to all subunits. In contrast to the MWC model, the KNF model of allostery requires asymmetric intermediates [121].

The need for asymmetric intermediates in the KNF model suggests once more that asymmetric intermediates are essential for the dynamic function of proteins. Indeed homooligomeric proteins are known to have functionally relevant asymmetric states apart from their symmetric states. One of these examples is the heat shock protein Chaperonin 60 that adopts a functionally relevant asymmetric configuration upon ATP binding 122. Furthermore, CorA, a multimeric $\mathrm{Mg}^{2+}$ channel, was found to abandon its symmetric closed state to open [123, 124].

For many structural analysis of a molecule its structure has to be stored, and thus each atom has to be assigned a label. For monomers and heterooligomers, conventions exist making the labeling of any atom unique. However, for homooligomers the subunits are identical, what results in ambigous subunit labels. If the asymmetry of a system is the result of a transition from a symmetric to an asymmetric state, it can take place in any of the subunits. In MD simulations, the ambiguity in subunit labeling challenges the analysis of these transitions because most analysis tools work on ensembles. As the asymmetry will spontaneously occur in varying subunits, the ensemble averaging will misleadingly show symmetric transition. To overcome this challenge, a thorough understanding of the occurance symmetry and 
asymmetry is required. A first step towards it is its quantification. The contribution of the subunits to the overall asymmetry is of special interest as it shows how the individual subunits behave and consequently can resolve the ambiguity. To label subunits unambiguously, the asymmetry contribution of each subunit can be used to have the major asymmetry consistently occurring in the same subunit.

Fully symmetric structures are unfavorable in entropy as well in interaction enthalpy of the contact zones of the multimers. Thus symmetry is unlikely if it is not enforced [125], because statistical asymmetry will arise due to thermal fluctuations, unrelated to protein function. As the interest in analyzing proteins focuses on their function, it remains obvious to identify asymmetry related to function. However, the availability of additional information about the protein function is required.

To analyze and quantify structural (a)symmetry, the Continuous Symmetry Measure (CSM) was developed by Zabrodsky et al. [126, 127], extended to incorporate further measures such as local symmetries $[125]$ and applied to various systems 125, 128. In this study we present and extension to CSM calculating subunit contributions to the overall asymmetry.

Naturally, the CSM algorithm is unable to distinguish functional from statistical asymmetry. Therefore, we extended the PLS-FMA algorithm 72,73 to quantify functional asymmetry. PLS-FMA is able to predict functionally relevant motions from an ensemble of structures such as trajectories of a MD simulation. The inclusion of the algorithm gives us the Functional Asymmetry MEasure (FAME) which describes the asymmetry along the functionally relevant motions identified by PLS-FMA. An implementation of the algorithms able to process MD simulation trajectories is available online at the website https:github.com/jtbrennecke/asympy. For a proof of principle, we applied CSM to modified KcsA and FAME to TREK2 simulations with artificially introduced asymmetry. We demonstrate the ability of both algorithms to recover the introduced asymmetry. Using FAME, the heat triggered unfolding of Transthyretin is studied using contact data as a functional property. The agreement of the subunit contributions with the individual subunit contact data demonstrates the ability of FAME to deal with more difficult functional modes.

With the new algorithms the symmetry of homooligomers can be quantified. 


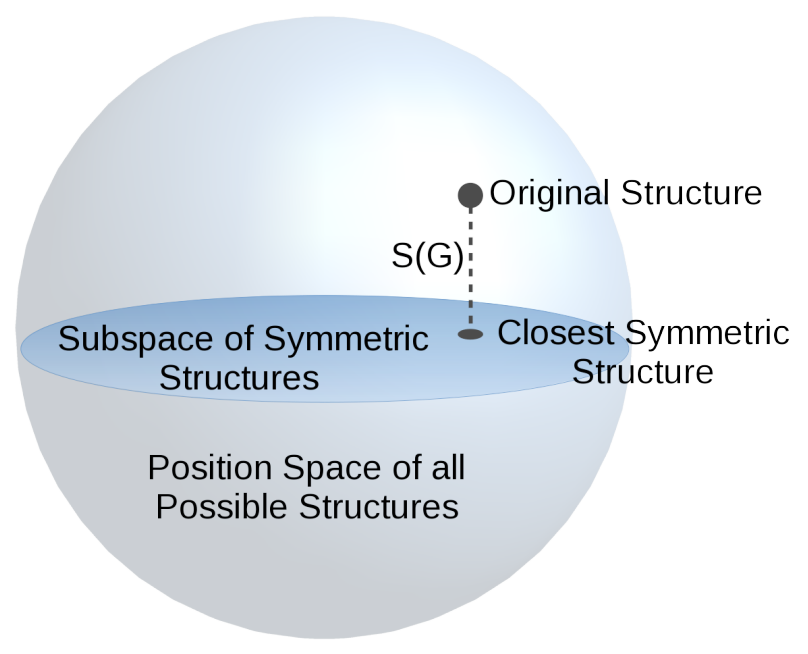

Figure 7.1.: CSM Position Space The studied original structure is projected onto its closest symmetric representation in position space. The normalized distance to this structure, indicated by the dotted line, is the CSM measure $(\mathrm{S}(\mathrm{G}))$.

\subsection{Theory}

In the following, the Continuous Symmetry Measure (CSM) will be introduced and extended to subunit contributions of asymmetry. For functionally relevant motions the Functional Asymmetry MEasure (FAME) will be developed. For both algorithms, an implementation is provided online at the website https://github.com/jtbrennecke/asympy.

This implementation works for rotational symmetries only as these are most common in proteins 114. However, the implementation can serve as a basis to extend it to other symmetries as well.

\subsubsection{Continuous Symmetry Measure (CSM)}

The Continuous Symmetry Measure (CSM) is the normalized distance $(S(G))$ of a structure to the nearest symmetric structure (see figure 7.1) 126]

$$
S(G)=\frac{100}{d^{2}} \sum_{i=1}^{N}\left|Q_{i}-Q_{i}^{s y m}\right|^{2} .
$$

$Q_{i}$ are the coordinates of the $\mathrm{i}^{\text {th }}$ atom of the protein with $N$ atoms. $Q_{i}^{s y m}$ is its corresponding symmetric structure and $d$ is a normalization factor taking the overall 
size of the protein $\left(d=\sqrt{\sum_{i=1}^{N}\left|Q_{i}\right|^{2}}\right)$ into account.

To determine the symmetric structure $\left(Q^{\text {sym }}\right)$ with the smallest distance to the original structure $(Q)$, rotations around the optimal rotation axis have to be performed. This axis can be identified analytically following the approach described by Pinsky et al. [127] Given the rotational axis, rotations according to the symmetry group of the protein are performed (see figure 7.2 ). Averaging over all rotations yields the closest symmetric structure 127 .

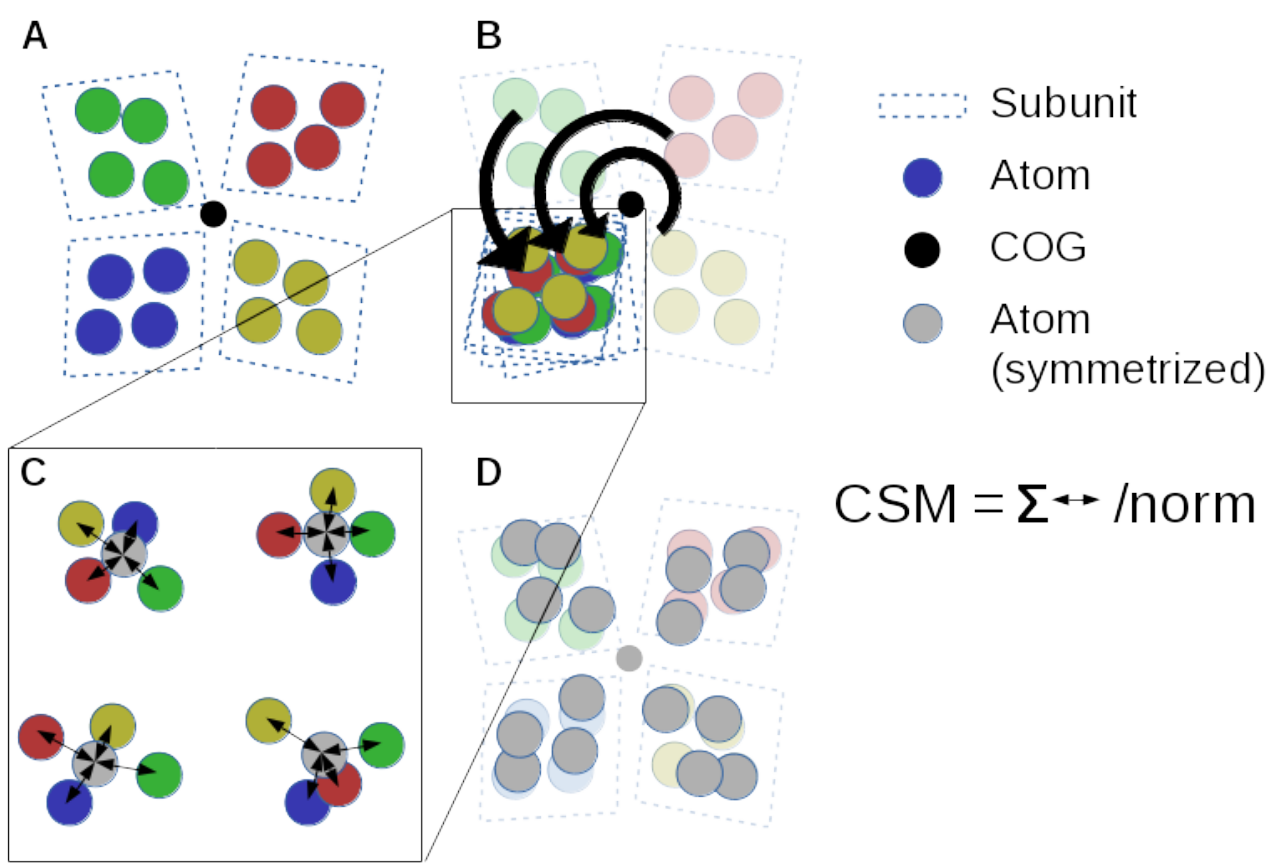

Figure 7.2.: Illustration of the CSM algorithm A visual introduction into the working of CSM. A A four fold symmetric protein (homo-tetramer, presented in 2D) of which the CSM measure is to be calculated. B Rotation around the center of geometry is applied such that all subunits are superimposed. In 3D, the rotation is performed around an axis which has to be determined previously. C The average position over the same atom in the different subunits is calculated. D Every subunit is averaged resulting in a symmetric structure.

For multimers consisting of more than two subunits, one subunit behaves most differently from the others. One example is the open state of the CorA $\mathrm{Mg}^{2+}$ channel in which four subunits form an almost symmetric structure while one subunit is moved outward 124. This distinction between the subunits is not captured in the current CSM algorithm. CSM only measures the asymmetry of 
the overall structure. To address the difference in asymmetry between the subunits we developed an extension to the CSM algorithm. As a result, a CSM measure for individual subunits is created what allows to distinguish the subunits based on their asymmetric behavior.

To access the CSM measure of each subunit $\left(S_{j}(G)\right.$ with $0<j \leq J$, with the number of subunits $J$ ), the calculation is split up into

$$
S_{j}(G)=\frac{100}{d^{2}} \sum_{i=j \cdot n+1}^{(j+1) \cdot n}\left|Q_{i}-Q_{i}^{s y m}\right|
$$

with $n=N / J$ the number of atoms per subunit. Note that the normalization factor is kept the same for the single subunits. As a result, $S_{j}(G)$ is not truly the CSM measure of each of the subunits, but rather a decomposition of the overall CSM measure into the contributions of each of the subunits. Thus the overall CSM measure is the result of a summation over the individual asymmetry measures of the subunits $\left(S(G)=\sum_{j=1}^{J} S_{j}(G)\right)$.

It is important to note that this additional decomposition is not meaningful for a dimer. For a dimer, a symmetric structure can be constructed that is the average of the two subunits. Thus the contribution of each of the subunits to the overall CSM measure is the same.

\subsubsection{Functional Asymmetry MEasure (FAME)}

Whereas the CSM method can be applied to quantify asymmetry in a system, using it, we are unable to distinguish between thermal fluctuations and functionally relevant asymmetry. Therefore, to distinguish functionally relevant from random asymmetry, we developed the functional asymmetry measure (FAME). The distinction is achieved by first determining the contributions of each subunit to a relevant functional motion of the protein. Subsequently, the information on the asymmetry is extracted by comparing the contributions of the subunits. One subunit can contribute significantly more than the others only if the motion described by the contributions is asymmetric. In contrast, if two subunits contribute similarly in respect to the same motion, we conclude the dynamics of the protein are symmetric.

To separate the functional motions from thermal fluctuations, we extended the PLS-FMA analysis 72,73 by FAME. Using PLS-FMA we try to extract the functional motions of a protein. A good estimate of the functional motion is often the maximally correlated motion (reference motion) to a given one dimensional functional property $(f)$. An example of this property could be the distance of two atoms in a binding site of an enzyme. As a result, PLS-FMA (and also FAME) requires a suitable functional property which can be challenging to find. 
In FAME, this functional property is used to calculate the contributions of the individual subunits to the reference motion and to extract the asymmetry by their relation to each other. To relate the contributions of the individual subunits to the (a)symmetry, they have to be calculated with respect to a symmetric reference motion. As a counter example: assuming an asymmetric reference motion, if two subunits would contribute equally, they could not be assumed to be symmetric as the reference motion of the individual subunits would describe different motions. However, as described before, the relation of contributions is supposed to describe the asymmetry i.e. the same contribution is defined to be symmetric. Thus the contributions have to be calculated along a symmetric reference motion. We construct the symmetric reference by permuting the monomers.

In mathematical terms, PLS-FMA is used to predict the functional property ( $\underline{\mathrm{f}}$ ) from a structural ensemble (e.g. a MD trajectory) $(\underline{\underline{X}})$ by determining the coefficients $(\beta)$ in the equation

$$
\underline{f}=\underline{\underline{X}} \underline{\beta}+\underline{\epsilon}
$$

to minimize the sum of errors $\left(\epsilon^{2}\right)$. Note that $f$ is a vector containing the unidimensional functional property to be predicted for each of the structures in the ensemble. The structural ensemble is summarized in the matrix $\underline{\underline{X}}$ containing the position of each atom for any structure of the ensemble.

To quantify asymmetry using PLS-FMA, the contributions of the subunits are separately analyzed. To separate the contributions, the coefficient vector $\beta$ is split into the individual subunits. In mathematical terms

$$
\underline{\tilde{f}}_{j}=\underline{\underline{X}} \underline{\beta}_{j}
$$

where $\underline{\tilde{f}}_{j}$ is the prediction of the contribution to the overall functional value $\underline{f}$ for the subunit $j$. The $i^{\text {th }}$ component of the vector $\underline{\beta}_{j}$ is given by

$$
\underline{\beta}_{j, i}= \begin{cases}\beta_{i} & \text { if } j \cdot 3 n \leq i<(j+1) \cdot 3 n \\ 0 & \text { otherwise }\end{cases}
$$

with $n$ denoting the number of atoms per subunit. Note that the coordinates of the structure are resorted to fit the $3 n$ coordinates into a one dimensional vector. The overall functional property $(\underline{\tilde{f}})$ is the sum over all contributions $\left(\underline{f}_{j}\right)$. As a result, the contribution of each of the subunits $\underline{C}_{j}$ to the overall functional property $\underline{f}$ can be estimated as $\underline{C}_{j}=\underline{\tilde{f}}_{j} / \underline{\tilde{f}}$. 

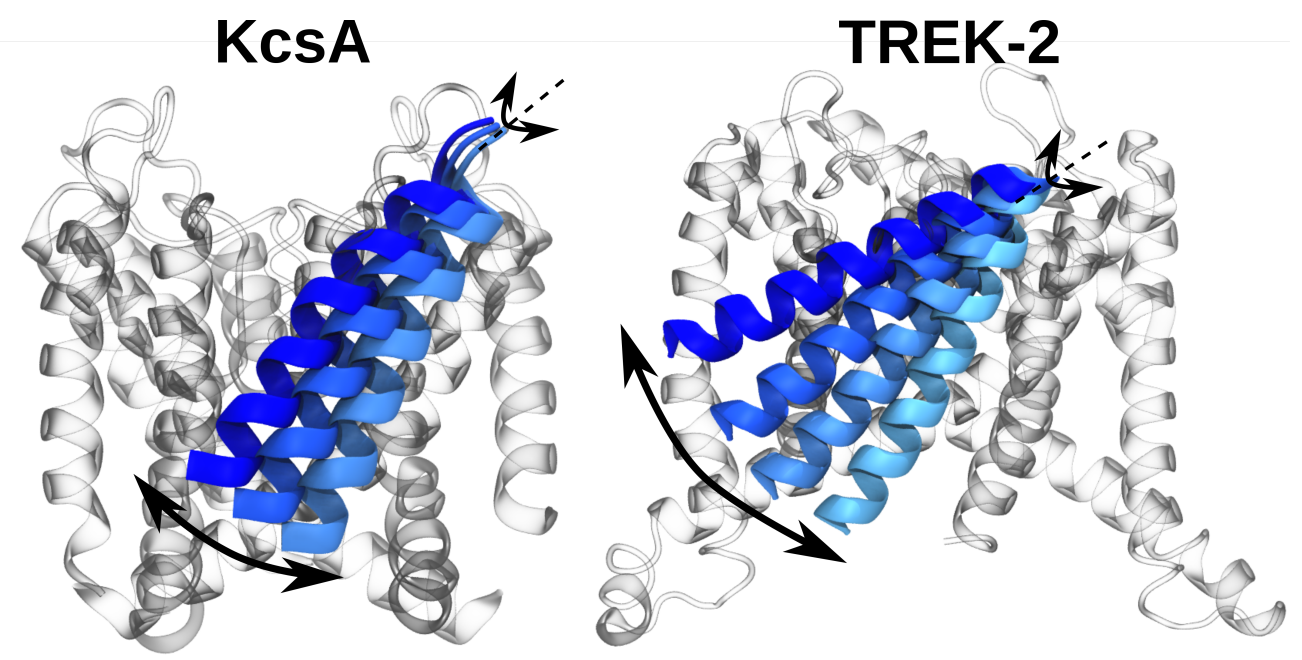

Figure 7.3.: Artificially Introduced Motion For two different test systems, the static KcsA channel and simulations of the TREK-2 channel, manipulations on outer helices are performed. They are rotated around a hinge point at their origin. These systems are later used to illustrate the analysis of (a)symmetry by CSM and FAME.

To interpret the result, the expectation on $\underline{C}_{j}$ needs to be understood for both asymmetric and symmetric subunits. If the subunits are symmetric, the contribution of each is expected to be $1 / J$ ( $J=N r$. of subunits). However, if they are asymmetric, the contributions deviate from $1 / J$ until they reach either zero or one. Zero corresponds to no contribution of the subunit whereas a value of one corresponds to a unique contribution of the subunit to the functional motion. By construction, $\underline{C}_{j}$ is normed to $\sum_{j} \underline{C}_{j}=1$.

\subsubsection{Applications}

To test the algorithms and illustrate their use in analyzing (a)symmetry, three different test systems were used, i.e. KcsA, TREK-2 and TTR. The first system is the well studied KcsA channel [15] with a four fold symmetric crystal structure (PDB ID: 3f5w). To control its motion, a trajectory is created by duplicating the crystal structure 500 times. These 500 structures are supposed to represent five trajectories containing 100 frames each. For each of these five trajectories, one of the four subunits is selected randomly. In this subunit, a motion of the outer helices is artificially introduced (see figure 7.3). This motion is a rigid body rotation around the base of the helix. In each frame, the helix is rotated slightly as long as the angle stays in a certain range. The angle and the subunit which is altered are recorded and shown in figure 7.4. Thereby, a trajectory of a symmetric protein with a well defined asymmetric motion in one of the subunits is created. The RMSD of the resulting trajectory is shown in the figure A.1. 
The second test system is the eukaryotic mechanosensitive potassium channel TREK-2. Previously published equilibrium MD simulations of this homodimer without its asymmetric cap were used [129]. On top of the motions from the MD simulations a similar protocol as described before for KcsA is used (see figure 7.3). However, to make it more realistic a signal, the functional property, was not exclusively introduced in one subunit but was distributed over both subunits. A trace of values was created similar to the angle in KcsA reflecting the functional property $(f)$ for FAME. The trace was distributed between the two subunits according to a second trace with values between zero and one. For the helix angle of subunit one, the first trace was multiplied with the distribution value (contribution of subunit one) and the remaining helix angle (1-distribution) demonstrated the movement of the helix angle of the second subunit. Thus the contribution to the signal by each of the subunits represents the asymmetry. If both subunits have the same angle (contribution $=0.5$ ), the system is assumed to be symmetric. In contrast, if only the first (second) subunit contributes (contribution=1.0 (0.0)), the highest functional asymmetry is assumed for the system. Predicting the asymmetry of the system is the main goal of this algorithm. The comparison of the RMSD of the system before and after introducing the helix motion is illustrated in figure A.2. The third test system is the human transport protein Transthyretin (TTR). MD simulations of tetrameric TTR (PDB ID: 4pvm [130]) were carried out at $298 \mathrm{~K}$ and at $598 \mathrm{~K}$. Whereas simulations at $298 \mathrm{~K}$ showed a stable behaviour, we saw denaturation of the protein in simulations at $598 \mathrm{~K}$ with one of the monomers typically initializing the unfolding process. The progress of denaturation can be quantified by the number of native contacts inside the protein. The overall number of native contacts was quantified using the tool developed by Best et al. 131 Furthermore, FAME was used to determine the individual degree of denaturation of each subunit.

\subsection{Results}

To demonstrate the usage of CSM and FAME and the interpretation of their results, artificial systems of KcsA and TREK-2 with a known asymmetry were created and tested. The more challenging example is the unfolding process of the tetrameric protein TTR. Here, information about native contacts is used that also reveals the contribution of the subunits to test a more subtle relation between functional property and structure of the system. 


\subsubsection{CSM analysis of KcsA}

To test the ability of the CSM algorithm in predicting the contributions of different subunits, full control over the complete asymmetry in the test system is required. To achieve this, a symmetric crystal structure of the KcsA channel was used and an asymmetric motion was artificially introduced (see section Applications). This asymmetric motion represents the symmetry distortion by bending one helix (see figure 7.3 .

The CSM algorithm was applied to the created ensemble. The result is the total asymmetry of the protein, and we find it to be similar to the input asymmetry (see figure 7.4). However, the total asymmetry does not give any details on the asymmetry distribution over the different subunits. As seen from the input data, the signal (helix motion) is present in the different subunits which is the information reconstructed by the presented extension of the CSM algorithm. For the first part of the frames $(<1000)$, the highest contribution of the asymmetry is assigned to the second subunit, in which the signal was introduced. However, looking at the resulting asymmetry, other subunits contribute partially. The partial asymmetry in these is a result of the symmetric structure definition, which is constructed by averaging over the configuration of each subunit. Thus the subunit with the introduced signal contributes to the average structure also. As a result, some of the contributions to the asymmetry can be found in the subunits without the signal, which is not an error but rather the asymmetry definition.
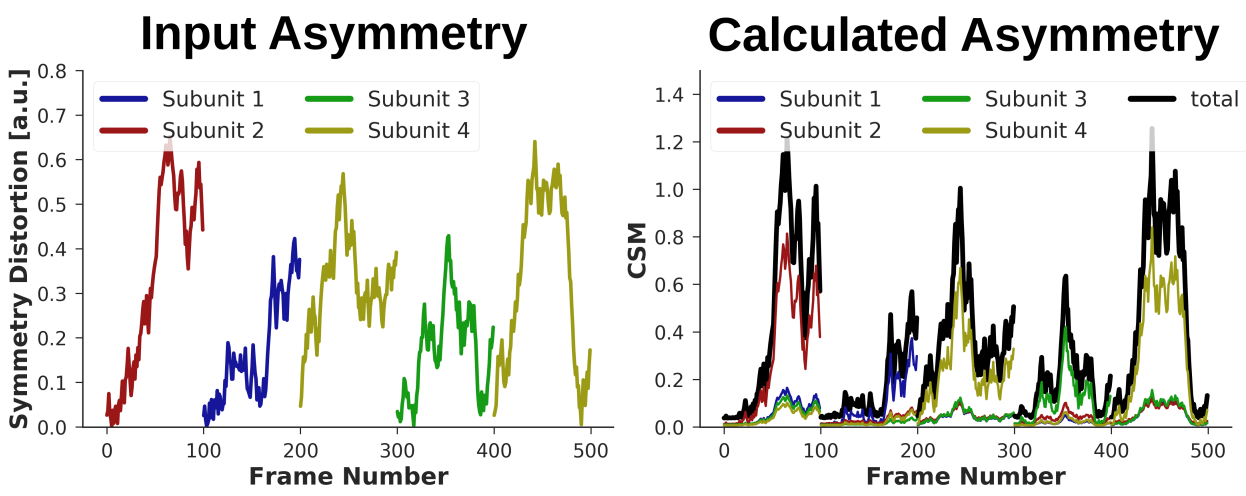

Figure 7.4.: CSM decomposition of KcsA The four fold symmetric KcsA channel (crystal structure) is used and a modification of a single helix is introduced. This helix is rotated by a given angle which distorts the symmetry of the structure. The distortion is shown in as the Input Asymmetry. Due to symmetry reasons, the distortion can be introduced in any of the four subunits which is indicated by the color. The asymmetry determined by the CSM algorithm is shown in Calculated Asymmetry. In addition to the total asymmetry score (black line), the contributions of the different subunits are shown (colored lines). 


\subsubsection{FAME analysis of TREK-2}

The advantage of the FAME algorithm compared to the CSM algorithm is its ability to distinguish between functional asymmetry and asymmetry due to thermal fluctuations. To test the prediction potential for functional asymmetry, a system containing functional as well as non-functional asymmetry was created. To this end, in the presented test system the functional asymmetry is added to a MD simulation trajectory of TREK-2 at room temperature. For the first analysis, the information about the helix angle was used directly as a functional property predicted by PLS-FMA. Secondly, the applicability of FAME to a more indirect functional property was tested (see section FAME analysis of TTR).

To this end, the overall helix angle was predicted by PLS-FMA. To use PLS-FMA, the optimal number of PLS components were evaluated first (refer to 73 for more details and see figure A.3. . Using the optimal number of components, the original input data was predicted with high precision (see figure 7.5, original data is predicted by PLS-FMA).

The FAME algorithm provides further information on the composition of the general prediction by PLS-FMA. The PLS-FMA prediction is decomposed into the contribution of the different subunits, which were used to calculate the functional asymmetry. The information about the asymmetry is stored in the input contribution as the overall signal is asymmetrically distributed over the two subunits. If the contributions are equally distributed between the two subunits, the signal is symmetric as dotted lines in figure 7.5 illustrate. On the contrary, if the contribution is above the dotted line, the signal is mainly found in subunit one, below the dotted line is a dominant signal in subunit two. This unequal distribution of the signal is the asymmetry. As can be seen in figure $7.5\left(C_{1}\right)$ the contribution predicted by the FAME algorithm is similar $\left(R^{2}=0.71\right)$ to the input contribution.

\subsubsection{FAME analysis of TTR}

The human Transthyretin (TTR) is a tetrameric protein transporting the hormone thyroxine and the retinol-binding protein 132,133 . The temperature triggered unfolding process of TTR was calculated. For the unfolding of a protein, the symmetry is not enforced and thus the unfolding process will start in one of the subunits and will likely be dominated by the unfolding of this subunit. Information on the unfolding process is gathered by calculating contact data (see section Applications). The contact data of the entire protein were used as functional input data (see figure A.4). The deviation of the different subunits of the tetramer to the average number of contacts is shown in figure 7.6. A deviation from the average number of contacts represents the asymmetry of one subunit. If all subunits would 


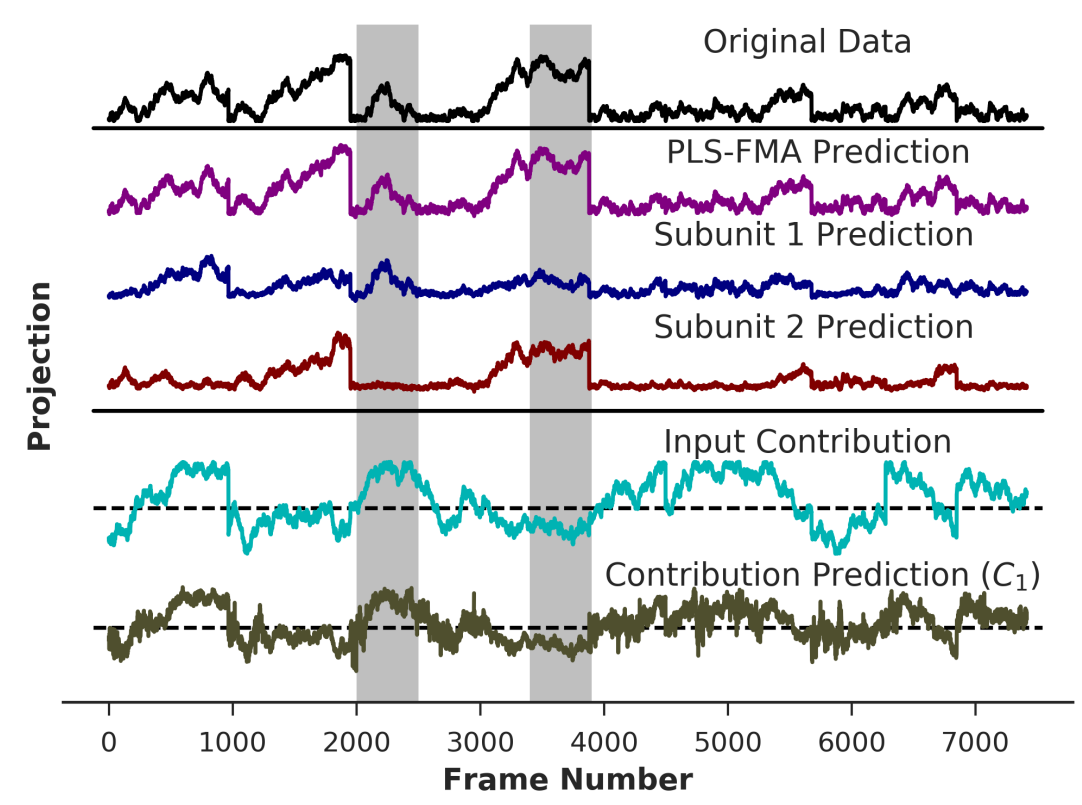

Figure 7.5.: Signal decomposition into subunit contributions The overall PLS-FMA input signal (Original Data) is predicted by the standard PLS-FMA algorithm (PLS-FMA Prediction). The FAME algorithm disassembled the signal into its contributions from the different subunits (Subunit 1/2 Prediction). The input asymmetry (Input Contribution) is predicted by the FAME algorithm (Contribution Prediction $\left(C_{1}\right)$ ) where the dotted lines represent equal contributions of the two subunits. The highlighted regions are illustrate the result of the algorithm particularly well. 

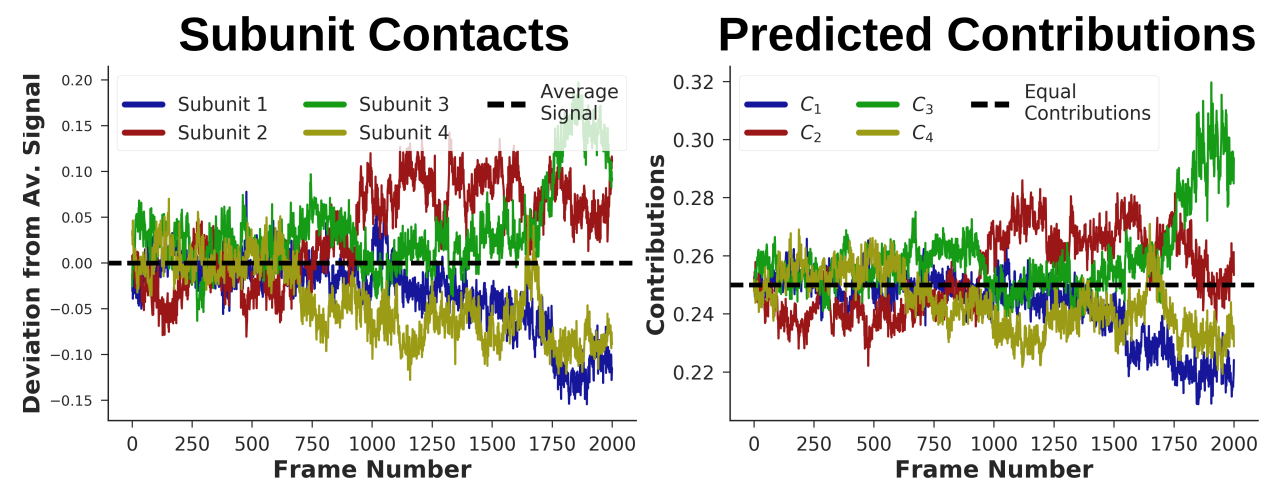

Figure 7.6.: TTR denaturation studied by FAME The Subunit Contacts are shown for each of the subunits. These are a measure of (a)symmetry across the subunits. We aim to predict the correct contributions of each of the subunit by FAME. The Predicted Contributions compare well to the subunit contacts which therefore shows FAMEs ability to retrieve the (a)symmetry of the system.

denature in the same way, the contact numbers of each of the subunits would be the same. Only if the denaturing process takes place in an asymmetric way, the contacts differ between the subunits. In figure 7.6 , the contributions $\left(C_{j}\right)$ of the different subunits to the overall denaturing process are predicted. The comparison of the input data to the calculated contributions reveals a significant agreement. Initially, the subunits behave very similarly. Only after around half of the trajectory, the subunits two and three show a higher contribution to the contact data than the other subunits. A comparable behaviour is apparent from the calculated subunit contributions. However, looking at the intermediate part, starting around frame 1000, the change in subunit two is more pronounced in the original data and almost returns to an equal contribution for the predicted data towards the end of the simulation.

\subsection{Discussion}

The aim of the presented research was to find suitable measures for (a)symmetry with and without functional relation. Application of our extension of CSM to KcsA show its ability to produce results in good agreement with the known input asymmetry on a subunit level. Comparing the input with regard to the subunits in which the helix angle motion is introduced and the results of the subunit information, the algorithm demonstrates its ability to correctly detect the subunit in which the change was introduced. It should be noted that the splitting of the CSM value into subunit contributions is only meaningful for oligomers with more 
than two subunits. In the case of a dimer, each of the subunits will have exactly the same contribution to the asymmetry as the closest fully symmetric structure is the average of the two.

The results of the TREK-2 FAME analysis demonstrate the ability of this algorithm to predict the contribution of each subunit accurately given a useful functional property. How the contributions and subunit predictions are related to the overall functional data can be readily derived from the highlighted region in the FAME algorithm results for TREK-2 (see figure 7.5). The first highlighted small peak in the original data, was predicted correctly by the PLS-FMA algorithm. Looking at the predictions for the individual subunits, it is obvious that the peak originates from a signal in subunit one whereas in subunit two, no signal can be seen. The input contributions show that at the creation time of the trajectory, the signal was mainly introduced into the first subunit, meaning that the helix of the first subunit was altered whereas the helix of the second subunit was kept in its original state. This asymmetry is what the algorithm is supposed to recover from the data. As can be seen for the given artificial data where the results of the algorithm can be compared to the input, the FAME algorithm recovers the contribution of the subunits $\left(C_{j}\right)$ well.

Another way to interpret the data is to consider what PLS-FMA does. PLSFMA creates a vector describing the motion of the protein most correlated with the functional data. Further, it projects the actual motion of the protein from the simulation along this vector. The contribution value shows at which position along this vector one subunit is located in reference to the other subunits. Therefore, the contribution value is equal to a functional asymmetry measure.

Similar to the KcsA channel, the information about the asymmetry of TTR was recovered. In this case, the functional value was decomposed into the contributions of the subunits. However, in most cases, such as the size of a cavity in the middle of a multimer, it is impossible to disassemble the functional value according to the contribution of the different subunits. In such cases, the presented methodology can contribute to a more complete understanding of a homooligomeric system.

Lastly, a comment on the possibility to combine both algorithms to estimate the overall asymmetry of a system compared to the functional asymmetry. If the CSM algorithm is used to compute the overall asymmetry of the system and the FAME algorithm is used to calculate the functional asymmetry, the difference of these two reveals statistical asymmetry of the system. Statistical asymmetry arises from a lack of enforcement of symmetry of the system and results mainly from thermal fluctuations.

Overall we could demonstrate our algorithms ability to quantify (a)symmetry with and without regard to functional relevance. 


\subsection{Conclusions}

Two methods were presented to analyze asymmetry. If additional information on a functional coordinate of interest is missing, a modified version of the CSM algorithm can be used [126, 127]. This version allows for the quantification of the overall system asymmetry as well as the quantification of the asymmetric contributions of the individual subunits.

If additional information on a functional property of the system is available, it allows for the use of PLS-FMA based FAME algorithm restricting asymmetry to functional motions only. The application of both algorithms to quantify asymmetry are shown and the interpretation of results is discussed. A comparison to the known input (a)symmetry revealed the ability of the algorithms to quantify asymmetry correctly.

The software used to perform the analysis is freely available online at the website https://github.com/jtbrennecke/asympy. It can be applied to simulations of rotational symmetric proteins without additional modifications. A more detailed step by step usage of the software is provided in the supplementary material of this publication.

\subsection{Acknowledgment}

We thankfully acknowledge Matteo Aldeghi for performing the MD simulations of TTR.

Funding awarded by the Deutsche Forschungsgesellschaft Sonderforschungsbereich 803: Project A03 to J.T.B. and B.L.d.G. is gratefully acknowledged. 



\section{Chapter 8}

\section{Summary and Conclusions}

To elucidate the mechanogating of $\mathrm{K} 2 \mathrm{P}$ channels, understanding of the gating mechanism is crucial as a first step. With this thesis, we focus on the gating mechanism of TRAAK and TREK-2 followed by mechanogating. Another relevant aspect in ion channel functionality is their structural symmetry, respectively their asymmetry. Therefore, two different algorithms allowing asymmetry quantification are presented in this thesis. The main conclusions from the applied theoretical approach are summarized in the following subchapters.

\subsection{Gating and Mechanogating}

Using MD simulations, we observed a change of conductance in K2P channels and thus were able to identify the gating mechanism. We identified the selectivity filter as the discriminating factor between a conductive and a non conductive channel and showed how its stability is influenced by the surrounding part of the structures of the channel in the 'up' and 'down' states. Whereas simulations of the up state displayed a more stable selectivity filter, simulations using the down crystal structure were prone to abandon the canonical configuration of the selectivity filter and adopted a flipped conformation. We suggest the selectivity filter flip as reason for the conductance change, as has also been speculated for C-type inactivation in other potassium channels such as KcsA.

Simulations conducted in a different setup for comparison and reduction of the force field bias, did not show spontaneous transitions between canonical and flipped selectivity filter configurations. However, the selectivity filter remained in a stable canonical configuration during up state simulations whereas flips occurred during the equilibration of the down state crystal structure and remained stable. The stability of the selectivity filter enabled us to exchange it between simulations of the two states and disentangle effects of the selectivity filter configuration from 
the overall state of the channel. Inserting the flipped selectivity filter into the up state configuration resulted in a non conductive channel whereas the down state could recover conductance partially by insertion of the canonical selectivity filter. This result supports gating by the selectivity filter rather than by a helix crossing gate.

After improved understanding of $\mathrm{K} 2 \mathrm{P}$ channel gating, we addressed the effect of membrane tension on channel conductance. Gating and mechanogating processes in $\mathrm{K} 2 \mathrm{P}$ channels share the common mechanism of selectivity filter gating. Channels simulated with increased membrane tension moved towards an up like configuration and subsequently showed increased conductance. As we found gating of $\mathrm{K} 2 \mathrm{P}$ channels to be influenced by the up/down state difference which is altered by membrane tension, we summarized our findings in a conclusive model of gating and mechanogating.

A selectivity filter gate theory is supported by multiple results from patch clamp experiments. Another gating theory is based on the lipid block hypothesis, evidenced by crystal structures of TRAAK showing a co-crystallized lipid chain inside the cavity. Additional experimental results of the same group support this hypothesis. To test the plausibility of lipid block gating, possibly in addition to selectivity filter gating, we simulated TRAAK with atoms of the lipid tail inside its cavity. However, the lipid left the cavity in free simulations rendering a lipid block mechanism unlikely.

\subsection{Quantification of Asymmetry}

Many computational analysis tools implicitly use ensemble averages to determine protein motions, e.g. Principal Component Analysis, and thus neglect asymmetry. Therefore, a solid understanding of asymmetry is required to correct for the neglect. As mentioned above, a first step towards understanding asymmetry of homooligomeric proteins is its quantification. Therefore, we developed two algorithms to quantify both their overall and functionally relevant asymmetry. We extended the existing Continuous Symmetry Measure (CSM) algorithm to determine the general asymmetry down to the subunit level but independent of the protein function. Additionally, we implemented the Functional Asymmetry MEasure (FAME) algorithm to calculate the asymmetry restricted to a functional reference motion and therefore particularly revealing the functional asymmetry.

We demonstrate the applicability of both algorithms in different test cases: The potassium channel KcsA was used to test the CSM algorithm and TREK-2 was used to test the FAME algorithm. As a more challenging test TTR unfolding 
was used. We recovered the asymmetry of the process using the FAME algorithm with information available on the overall unfolding process as additional information only. The calculations were in agreement with results obtained by additional information on the systems and are therefore suited to quantify asymmetry of homooligomeric proteins. However, application to further test systems are required to demonstrate the general applicability of the presented algorithms. 



\section{Chapter 9}

\section{Outlook}

To further contribute to understanding the K2P gating mechanism, future research could focus on the influence of the lower helix bundle on the selectivity filter. Our results suggest an influence of the surrounding part of the protein on the selectivity filter state to be part of the communication pathway from a membrane tension change to a conductance change. The major difference of the surrounding part is found in the lower helix bundle which is also identified to be altered by membrane tension. However, a full mechanistic understanding is not yet reached. A first step in this direction is the canonical correlation analysis (CCA) that can be used to identify maximally correlated motions of the lower helix bundle and the selectivity filter. A first assessment by a collaborator, using CCA, identified relevant residues located at the lower helix bundle of TREK-2. The configuration of these residues appears correlated to the selectivity filter state. Simulations with one of these residues replaced by Alanine, showed a change in selectivity filter stability accompanied by a change in conductance of TREK-2. However, the replaced residues do not explain the pathway by which the lower helix bundle influences the selectivity filter. Identification of the communication pathway should be part of future research.

To improve the interpretation of the asymmetry quantification algorithms, we suggest to apply the algorithms to additional systems. Identification of physiologically relevant asymmetric motions by the FAME algorithm could establish the presented methods in the field and advance the usage of these algorithms of MD users. A suitable system is the $\mathrm{Mg}^{2+}$ channel CorA which shows asymmetry in Cryo-EM structures for example 124. However, previous simulations showed additional bell-like deflection which are not supported experimentally and could reflect lacking protein stability in these simulations [123. For CorA, the bell-like deflection will likely interfere with the asymmetric closed to open transition rendering the overall interpretation of the data problematic and therefore the information gained 
by possible outcomes of these simulations should be assessed in advance.

Other potential test systems include anti-cooperative enzymes, as these systems show a well defined asymmetric transition. A potential candidate is the metabolic enzyme Glyceraldehyde-3-phosphate dehydrogenase (GADPH), showing anti-cooperativity at coenzyme binding $134-136$. For GADPH, two crystal structures are available at $3 \AA$ resolution $(2 \mathrm{X} 0 \mathrm{~N}, 2 \mathrm{HKI})$. The crystal structures might posses sufficient resolution and detail to simulate the enzyme. However, before simulating GADPH, an estimate of the transition time scales is required as simulations are currently limited to the millisecond time scale. For further asymmetric systems, refer to the review by Nagradova 137.

As computer power increases it will be more feasible to get to time scales of functionally asymmetric motions in the future. This increase in time scales will also allow future research on the gating mechanism of TREK/TRAAK channels in more detail as currently only few transitions of the selectivity filter state can be observed. 


\section{Bibliography}

[1] C.-C. Shieh, M. Coghlan, J. P. Sullivan, and M. Gopalakrishnan, "Potassium channels: molecular defects, diseases, and therapeutic opportunities," Pharmacological reviews, vol. 52, no. 4, pp. 557-594, 2000.

[2] M. E. Curran, I. Splawski, K. W. Timothy, G. M. Vincen, E. D. Green, and M. T. Keating, "A molecular basis for cardiac arrhythmia: Herg mutations cause long qt syndrome," Cell, vol. 80, no. 5, pp. 795-803, 1995.

[3] R. Reyes, F. Duprat, F. Lesage, M. Fink, M. Salinas, N. Farman, and M. Lazdunski, "Cloning and expression of a novel ph-sensitive two pore domain k+ channel from human kidney," Journal of Biological Chemistry, vol. 273, no. 47, pp. 30863-30869, 1998.

[4] F. M. Ashcroft and P. Rorsman, "Electrophysiology of the pancreatic $\beta$-cell," Progress in biophysics and molecular biology, vol. 54, no. 2, pp. 87-143, 1989.

[5] D. L. Browne, S. T. Gancher, J. G. Nutt, E. R. Brunt, E. A. Smith, P. Kramer, and M. Litt, "Episodic ataxia/myokymia syndrome is associated with point mutations in the human potassium channel gene, kcna1," Nature genetics, vol. 8, no. 2, p. 136, 1994.

[6] B. Hille, "Ionic channels of excitable membranes," tech. rep., Sinauer Associates Inc., 1992.

[7] Z. Xiao, P.-Y. Deng, L. Rojanathammanee, C. Yang, L. Grisanti, K. Permpoonputtana, D. Weinshenker, V. A. Doze, J. E. Porter, and S. Lei, "Noradrenergic depression of neuronal excitability in the entorhinal cortex via activation of TREK-2 $\mathrm{K}^{+}$channels," J. Biol. Chem., vol. 284, no. 16, pp. 10980-10991, 2009.

[8] H. Bang, Y. Kim, and D. Kim, "TREK-2, a new member of the mechanosensitive tandem-pore $\mathrm{K}^{+}$channel family," J. Biol. Chem., vol. 275, no. 23, pp. 17412-17419, 2000. 
[9] G. Sandoz, D. Douguet, F. Chatelain, M. Lazdunski, and F. Lesage, "Extracellular acidification exerts opposite actions on TREK1 and TREK2 potassium channels via a single conserved histidine residue," $P N A S$, vol. 106, no. 34, pp. 14628-14633, 2009.

[10] J. Noël, G. Sandoz, and F. Lesage, "Molecular regulations governing TREK and TRAAK channel functions," Channels, vol. 5, no. 5, pp. 402-409, 2011.

[11] P. Enyedi and G. Czirják, "Molecular background of leak $\mathrm{K}^{+}$currents: Twopore domain potassium channels," Physiol. Rev., vol. 90, no. 2, pp. 559-605, 2010 .

[12] S. G. Brohawn, Z. Su, and R. MacKinnon, "Mechanosensitivity is mediated directly by the lipid membrane in TRAAK and TREK1 $\mathrm{K}^{+}$channels," PNAS, vol. 111, no. 9, pp. 3614-3619, 2014.

[13] S. G. Brohawn, J. del Mármol, and R. MacKinnon, "Crystal structure of the human K2P TRAAK, a lipid- and mechano-sensitive $\mathrm{K}^{+}$ion channel," Science, vol. 335, no. 6067, pp. 436-441, 2012.

[14] Y. Zhou, J. H. Morais-Cabral, A. Kaufman, and R. MacKinnon, "Chemistry of ion coordination and hydration revealed by a $\mathrm{K}^{+}$; channel - fab complex at 2.0 A resolution," Nature, vol. 414, no. 6859, pp. 43-48, 2001.

[15] D. A. Köpfer, C. Song, T. Gruene, G. M. Sheldrick, U. Zachariae, and B. L. de Groot, "Ion permeation in $\mathrm{K}^{+}$channels occurs by direct coulomb knockon," Science, vol. 346, no. 6207, pp. 352-355, 2014.

[16] S. G. Brohawn, E. B. Campbell, and R. MacKinnon, "Physical mechanism for gating and mechanosensitivity of the human traak k+ channel," Nature, vol. 516, no. 7529, pp. 126-130, 2014.

[17] Y. Y. Dong, A. C. Pike, A. Mackenzie, C. McClenaghan, P. Aryal, L. Dong, A. Quigley, M. Grieben, S. Goubin, S. Mukhopadhyay, G. F. Ruda, M. V. Clausen, L. Cao, P. E. Brennan, N. A. Burgess-Brown, M. S. P. Sansom, S. J. Tucker, and E. P. Carpenter, "K2P channel gating mechanisms revealed by structures of TREK-2 and a complex with Prozac," Science, vol. 347, no. 6227, pp. 1256-1259, 2015.

[18] C. Heurteaux, G. Lucas, N. Guy, M. El Yacoubi, S. Thümmler, X.-D. Peng, F. Noble, N. Blondeau, C. Widmann, M. Borsotto, et al., "Deletion of the background potassium channel trek-1 results in a depression-resistant phenotype," Nat. Neurosci., vol. 9, no. 9, pp. 1134-1141, 2006. 
[19] L. E. Kennard, J. R. Chumbley, K. M. Ranatunga, S. J. Armstrong, E. L. Veale, and A. Mathie, "Inhibition of the human two-pore domain potassium channel, trek-1, by fluoxetine and its metabolite norfluoxetine," Br. J. Pharmacol., vol. 144, no. 6, pp. 821-829, 2005.

[20] M. Lolicato, P. Riegelhaupt, C. Arrigoni, K. Clark, and D. M. Jr., "Transmembrane helix straightening and buckling underlies activation of mechanosensitive and thermosensitive K2P channels," Neuron, vol. 84, no. 6, pp. 1198-1212, 2014.

[21] S. N. Bagriantsev, R. Peyronnet, K. A. Clark, E. Honore, and D. L. Minor, "Multiple modalities converge on a common gate to control K2P channel function," EMBO J., vol. 30, no. 17, pp. 3594-3606, 2011.

[22] P. L. Piechotta, M. Rapedius, P. J. Stansfeld, M. K. Bollepalli, G. Erhlich, I. Andres-Enguix, H. Fritzenschaft, N. Decher, M. S. Sansom, S. J. Tucker, and T. Baukrowitz, "The pore structure and gating mechanism of K2P channels," EMBO J., vol. 30, no. 17, pp. 3607-3619, 2011.

[23] M. Rapedius, M. R. Schmidt, C. Sharma, P. J. Stansfeld, M. S. Sansom, T. Baukrowitz, and S. J. Tucker, "State-independent intracellular access of quaternary ammonium blockers to the pore of TREK-1," Channels, vol. 6, no. 6, pp. 473-478, 2012.

[24] N. Zilberberg, N. Ilan, and S. A. Goldstein, "KCNKØ: opening and closing the 2-p-domain potassium leak channel entails "C-type" gating of the outer pore," Neuron, vol. 32, no. 4, pp. 635-648, 2001.

[25] A. Cohen, Y. Ben-Abu, and N. Zilberberg, "Gating the pore of potassium leak channels," European Biophysics Journal, vol. 39, no. 1, pp. 61-73, 2009.

[26] R. E. Hulse, J. R. Sachleben, P.-C. Wen, M. Moradi, E. Tajkhorshid, and E. Perozo, "Conformational dynamics at the inner gate of kcsa during activation," Biochemistry, vol. 53, no. 16, pp. 2557-2559, 2014.

[27] R.-G. Zhuo, P. Peng, X.-Y. Liu, H.-T. Yan, J.-P. Xu, J.-Q. Zheng, X.-L. Wei, and X.-Y. Ma, "Allosteric coupling between proximal C-terminus and selectivity filter is facilitated by the movement of transmembrane segment 4 in TREK-2 channel," Sci. Rep., vol. 6, p. 21248, 2016.

[28] J. P. Schrum, T. F. Zhu, and J. W. Szostak, "The origins of cellular life," Cold Spring Harbor perspectives in biology, vol. 2, no. 9, p. a002212, 2010. 
[29] I. Budin, A. Debnath, and J. W. Szostak, "Concentration-driven growth of model protocell membranes," Journal of the American Chemical Society, vol. 134, no. 51, pp. 20812-20819, 2012.

[30] B. D. Bennett, E. H. Kimball, M. Gao, R. Osterhout, S. J. Van Dien, and J. D. Rabinowitz, "Absolute metabolite concentrations and implied enzyme active site occupancy in escherichia coli," Nature chemical biology, vol. 5, no. 8, p. 593, 2009.

[31] C. Delamarche, D. Thomas, J.-P. Rolland, A. Froger, J. Gouranton, M. Svelto, P. Agre, and G. Calamita, "Visualization of aqpz-mediated water permeability in escherichia coli by cryoelectron microscopy," Journal of bacteriology, vol. 181, no. 14, pp. 4193-4197, 1999.

[32] I. R. Booth and P. Blount, "The MscS and MscL families of mechanosensitive channels act as microbial emergency release valves," J. Bacteriol., vol. 194, no. 18, pp. 4802-4809, 2012.

[33] D. Schmidt, J. del Mármol, and R. MacKinnon, "Mechanistic basis for low threshold mechanosensitivity in voltage-dependent k+ channels," Proceedings of the National Academy of Sciences, vol. 109, no. 26, pp. 10352-10357, 2012 .

[34] I. R. Booth, S. Miller, A. Müller, and L. Lehtovirta-Morley, "The evolution of bacterial mechanosensitive channels," Cell Calcium, vol. 57, no. 3, pp. 140150, 2015.

[35] S. Ranade, R. Syeda, and A. Patapoutian, "Mechanically activated ion channels," Neuron, vol. 87, no. 6, pp. 1162 - 1179, 2015.

[36] S. Sukharev and F. Sachs, "Molecular force transduction by ion channels diversity and unifying principles," J. Cell Sci., vol. 125, no. 13, pp. 30753083, 2012.

[37] S.-H. Woo, E. A. Lumpkin, and A. Patapoutian, "Merkel cells and neurons keep in touch," Trends in cell biology, vol. 25, no. 2, pp. 74-81, 2015.

[38] M. Beurg, R. Fettiplace, J.-H. Nam, and A. J. Ricci, "Localization of inner hair cell mechanotransducer channels using high-speed calcium imaging," Nature neuroscience, vol. 12, no. 5, p. 553, 2009.

[39] J. Hu and G. R. Lewin, "Mechanosensitive currents in the neurites of cultured mouse sensory neurones," The Journal of physiology, vol. 577, no. 3, pp. 815$828,2006$. 
[40] J. Hao and P. Delmas, "Multiple desensitization mechanisms of mechanotransducer channels shape firing of mechanosensory neurons," Journal of Neuroscience, vol. 30, no. 40, pp. 13384-13395, 2010.

[41] F. Rugiero, L. J. Drew, and J. N. Wood, "Kinetic properties of mechanically activated currents in spinal sensory neurons," The Journal of physiology, vol. 588, no. 2, pp. 301-314, 2010.

[42] P. Delmas, J. Hao, and L. Rodat-Despoix, "Molecular mechanisms of mechanotransduction in mammalian sensory neurons," Nature Reviews Neuroscience, vol. 12, no. 3, p. 139, 2011.

[43] Z. Yan, W. Zhang, Y. He, D. Gorczyca, Y. Xiang, L. E. Cheng, S. Meltzer, L. Y. Jan, and Y. N. Jan, "Drosophila NOMPC is a mechanotransduction channel subunit for gentle-touch sensation," Nature, vol. 493, no. 7431, pp. 221-225, 2013.

[44] B. Coste, J. Mathur, M. Schmidt, T. J. Earley, S. Ranade, M. J. Petrus, A. E. Dubin, and A. Patapoutian, "Piezo1 and Piezo2 are essential components of distinct mechanically activated cation channels," Science, vol. 330, no. 6000, pp. 55-60, 2010.

[45] Z. Su, A. Anishkin, C. Kung, and Y. Saimi, "The core domain as the force sensor of the yeast mechanosensitive TRP channel," The Journal of general physiology, vol. 138, no. 6, pp. 627-640, 2011.

[46] J. Árnadóttir and M. Chalfie, "Eukaryotic mechanosensitive channels," Annual review of biophysics, vol. 39, pp. 111-137, 2010.

[47] B. Martinac, J. Adler, and C. Kung, "Mechanosensitive ion channels of e. coli activated by amphipaths," Nature, vol. 348, no. 6298, pp. 261-263, 1990.

[48] S. I. Sukharev, P. Blount, B. Martinac, and C. Kung, "Mechanosensitive channels of escherichia coli: the MscL gene, protein, and activities," Annu. Rev. Physiol., vol. 59, no. 1, pp. 633-657, 1997.

[49] J. Teng, S. Loukin, A. Anishkin, and C. Kung, "The force-from-lipid (FFL) principle of mechanosensitivity, at large and in elements," Pflugers Arch., vol. 467, no. 1, pp. 27-37, 2015.

[50] M. D. Edwards, Y. Li, S. Kim, S. Miller, W. Bartlett, S. Black, S. Dennison, I. Iscla, P. Blount, J. U. Bowie, et al., "Pivotal role of the glycine-rich tm3 helix in gating the mscs mechanosensitive channel," Nature Structural and Molecular Biology, vol. 12, no. 2, p. 113, 2005. 
[51] C. Kung, "A possible unifying principle for mechanosensation," Nature, vol. 436, no. 7051, pp. 647-654, 2005.

[52] F. Maingret, M. Fosset, F. Lesage, M. Lazdunski, and E. HonorÃ@), "TRAAK is a mammalian neuronal mechano-gated K+channel," J. Biol. Chem., vol. 274, no. 3, pp. 1381-1387, 1999.

[53] E. Neher and B. Sakmann, "The patch clamp technique," Scientific American, vol. 266, no. 3, pp. 44-51, 1992.

[54] J. J. Clare, "Targeting ion channels for drug discovery," Discovery medicine, vol. 9, no. 46, pp. 253-260, 2010.

[55] R. O. Dror, M. O. Jensen, D. W. Borhani, and D. E. Shaw, "Exploring atomic resolution physiology on a femtosecond to millisecond timescale using molecular dynamics simulations," J Gen Physiol, vol. 135(6), pp. 555-562, 2010 .

[56] P. Carloni, U. Rothlisberger, and M. Parrinello, "The role and perspective of ab initio molecular dynamics in the study of biological systems," Accounts of Chemical Research, vol. 35, no. 6, pp. 455-464, 2002.

[57] B. Hess, H. Bekker, H. J. C. Berendsen, and J. G. E. M. Fraaije, "Lincs: A linear constraint solver for molecular simulations," J Comput Chem, vol. 18, no. 12, pp. 1463-1472, 1997.

[58] J. Ryckaert, G. Ciccotti, and H. Berendsen, "Numerical integration of the cartesian equations of motion of a system with constraints: molecular dynamics of n-alkanes," J Comp Phys, vol. 23, no. 3, pp. 327-341, 1977.

[59] T. Darden, D. York, and L. Pedersen, "Particle mesh ewald: An n·log(n) method for ewald sums in large systems," J Chem Phys, vol. 98, p. 10089, 1993.

[60] S. Rauscher, V. Gapsys, M. J. Gajda, M. Zweckstetter, B. L. de Groot, and H. Grubmüller, "Structural ensembles of intrinsically disordered proteins depend strongly on force field: a comparison to experiment," Journal of chemical theory and computation, vol. 11, no. 11, pp. 5513-5524, 2015.

[61] H. J. C. Berendsen and W. F. Van Gunsteren, Molecular Dynamics Simulations: Techniques and Approaches, pp. 475-500. Dordrecht: Springer Netherlands, 1984.

[62] M. Parrinello and A. Rahman, "Polymorphic transitions in single crystals: A new molecular dynamics method," J Appl Phys, vol. 52, p. 7182, 1981. 
[63] G. Bussi, D. Donadio, and M. Parrinello, "Canonical sampling through velocity rescaling," J Chem Phys, vol. 126, p. 014101, Jan. 2007.

[64] H. M. Senn and W. Thiel, "Qm/mm methods for biomolecular systems," Angewandte Chemie International Edition, vol. 48, no. 7, pp. 1198-1229, 2009.

[65] J. W. Ponder and D. A. Case, "Force fields for protein simulations," $A d v$ Protein Chem, vol. 66, pp. 27-+, 2003.

[66] A. Warshel and S. T. Russell, "Calculations of electrostatic interactions in biological - systems and in solutions," Q Rev Biophys, vol. 17, no. 3, pp. 283$422,1984$.

[67] F. S. Lee, Z. T. Chu, and A. Warshel, "Microscopic and semimicroscopic calculations of electrostatic energies in proteins by the polaris and enzymix programs," J Comput Chem, vol. 14, pp. 161-185, Feb. 1993.

[68] C. Kutzner, H. Grubmüller, B. L. De Groot, and U. Zachariae, "Computational electrophysiology: the molecular dynamics of ion channel permeation and selectivity in atomistic detail," Biophysical journal, vol. 101, no. 4, pp. 809-817, 2011.

[69] B. Roux, "The membrane potential and its representation by a constant electric field in computer simulations," Biophysical journal, vol. 95, no. 9, pp. 4205-4216, 2008.

[70] M. Neumann, "Dipole moment fluctuation formulas in computer simulations of polar systems," Molecular Physics, vol. 50, no. 4, pp. 841-858, 1983.

[71] R. A. Böckmann, B. L. De Groot, S. Kakorin, E. Neumann, and H. Grubmüller, "Kinetics, statistics, and energetics of lipid membrane electroporation studied by molecular dynamics simulations," Biophysical journal, vol. 95, no. 4, pp. 1837-1850, 2008.

[72] J. S. Hub and B. L. de Groot, "Detection of functional modes in protein dynamics," Plos Comput Biol, vol. 5, p. e1000480, Aug. 2009.

[73] T. Krivobokova, R. Briones, J. S. Hub, A. Munk, and B. L. de Groot, "Partial least-squares functional mode analysis: Application to the membrane proteins aqp1, aqy1, and clc-ec1," Biophys J, vol. 103, pp. 786-796, Aug. 2012 . 
[74] M. Schewe, E. Nematian-Ardestani, H. Sun, M. Musinszki, S. Cordeiro, G. Bucci, B. L. de Groot, S. J. Tucker, M. Rapedius, and T. Baukrowitz, "A non-canonical voltage-sensing mechanism controls gating in $\mathrm{K} 2 \mathrm{P} \mathrm{K}^{+}$channels," Cell, vol. 164, no. 5, pp. 937-949, 2016.

[75] B. Hess, C. Kutzner, D. van der Spoel, and E. Lindahl, "Gromacs 4.0: algorithms for highly efficient, load-balanced, and scalable molecular simulation," J Chem Theory Comput., vol. 4, pp. 435-447, 2008.

[76] V. Hornak, R. Abel, A. Okur, B. Strockbine, A. Roitberg, and C. Simmerling, "Comparison of multiple amber force fields and development of improved protein backbone parameters," Proteins: Struct., Funct., Bioinf., vol. 65, no. 3, pp. 712-725, 2006.

[77] H. Berendsen, J. Grigera, and T. Straatsma, "The missing term in effective pair potentials," J. Phys. Chem., vol. 91, no. 24, pp. 6269-6271, 1987.

[78] I. S. Joung and T. E. Cheatham III, "Determination of alkali and halide monovalent ion parameters for use in explicitly solvated biomolecular simulations," J. Phys. Chem. B, vol. 112, no. 30, pp. 9020-9041, 2008.

[79] O. Berger, O. Edholm, and F. Jähnig, "Molecular dynamics simulations of a fluid bilayer of dipalmitoylphosphatidylcholine at full hydration, constant pressure, and constant temperature," Biophys. J., vol. 72, no. 5, pp. 20022013, 1997.

[80] M. Bachar, P. Brunelle, D. P. Tieleman, and A. Rauk, "Molecular dynamics simulation of a polyunsaturated lipid bilayer susceptible to lipid peroxidation," J. Phy. Chem. B, vol. 108, no. 22, pp. 7170-7179, 2004.

[81] A. Cordomí, G. Caltabiano, and L. Pardo, "Membrane protein simulations using amber force field and berger lipid parameters," J. Chem. Theory Comput., vol. 8, no. 3, pp. 948-958, 2012.

[82] T. Darden, D. York, and L. Pedersen, "Particle mesh ewald: An N log (N) method for ewald sums in large systems," J. Chem. Phys., vol. 98, no. 12, pp. 10089-10092, 1993.

[83] U. Essmann, L. Perera, M. L. Berkowitz, T. Darden, H. Lee, and L. G. Pedersen, "A smooth particle mesh ewald method," J. Chem. Phys., vol. 103, no. 19, pp. 8577-8593, 1995.

[84] G. Bussi, D. Donadio, and M. Parrinello, "Canonical sampling through velocity rescaling," J. Chem. Phys., vol. 126, no. 1, p. 014101, 2007. 
[85] H. J. Berendsen, J. v. Postma, W. F. van Gunsteren, A. DiNola, and J. Haak, "Molecular dynamics with coupling to an external bath," J. Chem. Phys., vol. 81, no. 8, pp. 3684-3690, 1984.

[86] B. Hess, H. Bekker, H. J. Berendsen, and J. G. Fraaije, "Lincs: a linear constraint solver for molecular simulations," J. Comput. Chem., vol. 18, no. 12 , pp. 1463-1472, 1997.

[87] K. A. Feenstra, B. Hess, and H. J. Berendsen, "Improving effciency of large timescale molecular dynamics simulations of hydrogen-rich systems," J. Comput. Chem., vol. 20, pp. 786-798, 1999.

[88] D. van der Spoel, E. Lindahl, B. Hess, G. Groenhof, A. E. Mark, and H. J. C. Berendsen, "GROMACS: fast, flexible and free," J. Comput. Chem., vol. 26, pp. 1701-1718, 2005.

[89] A. Amadei, A. B. M. Linssen, and H. J. C. Berendsen, "Essential dynamics of proteins," Proteins, vol. 17, pp. 412-425, Dec. 1993.

[90] P. Aryal, V. Jarerattanachat, M. V. Clausen, M. Schewe, C. McClenaghan, L. Argent, L. J. Conrad, Y. Y. Dong, A. C. Pike, E. P. Carpenter, T. Baukrowitz, M. S. Sansom, and S. J. Tucker, "Bilayer-mediated structural transitions control mechanosensitivity of the TREK-2 K2P channel," Structure, vol. 25, no. 5, pp. 708-718, 2017.

[91] B. Katz, "Action potentials from a sensory nerve ending," J. Physiol., vol. 111, no. 3-4, pp. 248-260, 1950.

[92] J. Gullingsrud, D. Kosztin, and K. Schulten, "Structural determinants of MscL gating studied by molecular dynamics simulations," Biophys. J., vol. 80, no. 5, pp. 2074-2081, 2001.

[93] M. Sotomayor and K. Schulten, "Molecular dynamics study of gating in the mechanosensitive channel of small conductance MscS," Biophys. J., vol. 87, no. 5, pp. 3050-3065, 2004.

[94] C. Acosta, L. Djouhri, R. Watkins, C. Berry, K. Bromage, and S. N. Lawson, "TREK2 expressed selectively in IB4-binding C-fiber nociceptors hyperpolarizes their membrane potentials and limits spontaneous pain," J. Neurosci., vol. 34, no. 4, pp. 1494-1509, 2014.

[95] V. Pereira, J. Busserolles, M. Christin, M. Devilliers, L. Poupon, W. Legha, A. Alloui, Y. Aissouni, E. Bourinet, F. Lesage, A. Eschalier, M. Lazdunskide, and J. Noël, "Role of the TREK2 potassium channel in cold and warm 
thermosensation and in pain perception," PAIN®, vol. 155 , no. 12 , pp. 25342544, 2014.

[96] Z. Es-Salah-Lamoureux, D. F. Steele, and D. Fedida, "Research into the therapeutic roles of two-pore-domain potassium channels," Trends Pharmacol. Sci., vol. 31, no. 12, pp. 587-595, 2010.

[97] M. P. Harrigan, K. A. McKiernan, V. Shanmugasundaram, R. A. Denny, and V. S. Pande, "Markov modeling reveals novel intracellular modulation of the human TREK-2 selectivity filter," Sci. Rep., vol. 7, no. 1, p. 632, 2017.

[98] D. van der Spoel, E. Lindahl, B. Hess, A. R. van Buuren, E. Apol, P. J. Meulenhoff, D. P. Tieleman, A. L. T. M. Sijbers, K. A. Feenstra, R. van Drunen, and H. J. C. Berendsen, Gromacs User Manual version 5.0. www.gromacs.org, 2014.

[99] C. S. Soto, M. Fasnacht, J. Zhu, L. Forrest, and B. Honig, "Loop modeling: Sampling, filtering, and scoring," Proteins: Struct., Funct., Bioinf., vol. 70, no. 3, pp. 834-843, 2008.

[100] M. G. Wolf, M. Hoefling, C. Aponte-Santamaría, H. Grubmüller, and G. Groenhof, "g_membed: Efficient insertion of a membrane protein into an equilibrated lipid bilayer with minimal perturbation," J. Comput. Chem., vol. 31, no. 11, pp. 2169-2174, 2010.

[101] J. B. Klauda, R. M. Venable, J. A. Freites, J. W. O’ Connor, D. J. Tobias, C. Mondragon-Ramirez, I. Vorobyov, A. D. MacKerell Jr, and R. W. Pastor, "Update of the CHARMM all-atom additive force field for lipids: validation on six lipid types," J. Phys. Chem. B, vol. 114, no. 23, p. 7830, 2010.

[102] S. Jo, T. Kim, V. G. Iyer, and W. Im, "CHARMM-GUI: a web-based graphical user interface for CHARMM," J. Comput. Chem., vol. 29, no. 11, pp. 1859-1865, 2008.

[103] E. L. Wu, X. Cheng, S. Jo, H. Rui, K. C. Song, E. M. Dávila-Contreras, Y. Qi, J. Lee, V. Monje-Galvan, R. M. Venable, J. B. Klauda, and W. Im, "CHARMM-GUI membrane builder toward realistic biological membrane simulations," J. Comput. Chem., vol. 35, no. 27, pp. 1997-2004, 2014.

[104] S. Jo, J. B. Lim, J. B. Klauda, and W. Im, "CHARMM-GUI membrane builder for mixed bilayers and its application to yeast membranes," Biophys. J., vol. 97, no. 1, pp. 50-58, 2009. 
[105] B. R. Brooks, C. L. Brooks, A. D. Mackerell, L. Nilsson, R. J. Petrella, B. Roux, Y. Won, G. Archontis, C. Bartels, S. Boresch, A. Caflisch, L. Caves, Q. Cui, A. R. Dinner, M. Feig, S. Fischer, J. Gao, M. Hodoscek, W. Im, K. Kuczera, T. Lazaridis, J. Ma, V. Ovchinnikov, E. Paci, R. W. Pastor, C. B. Post, J. Z. Pu, M. Schaefer, B. Tidor, R. M. Venable, H. L. Woodcock, X. Wu, W. Yang, D. M. York, and M. Karplus, "CHARMM: the biomolecular simulation program," J. Comput. Chem., vol. 30, no. 10, pp. 1545-1614, 2009.

[106] J. Lee, X. Cheng, J. M. Swails, M. S. Yeom, P. K. Eastman, J. A. Lemkul, S. Wei, J. Buckner, J. C. Jeong, Y. Qi, S. Jo, V. S. Pande, D. A. Case, C. L. Brooks, A. D. MacKerell, J. B. Klauda, and W. Im, "CHARMMGUI input generator for NAMD, GROMACS, AMBER, OpenMM, and CHARMM/OpenMM simulations using the CHARMM36 additive force field," J. Chem. Theory Comput., vol. 12, no. 1, pp. 405-413, 2016.

[107] B. Roux, "Influence of the membrane potential on the free energy of an intrinsic protein," Biophys. J., vol. 73, no. 6, pp. 2980-2989, 1997.

[108] C. Kutzner, H. Grubmüller, B. L. De Groot, and U. Zachariae, "Computational electrophysiology: the molecular dynamics of ion channel permeation and selectivity in atomistic detail," Biophys. J., vol. 101, no. 4, pp. 809-817, 2011.

[109] C. Gnatenco, J. Han, A. K. Snyder, and D. Kim, "Functional expression of TREK-2 $\mathrm{K}^{+}$channel in cultured rat brain astrocytes," Brain Res., vol. 931, no. 1, pp. 56-67, 2002.

[110] M. Lolicato, C. Arrigoni, T. Mori, Y. Sekioka, C. Bryant, K. A. Clark, and D. L. Minor Jr, "K2P2. 1 (TREK-1)-activator complexes reveal a cryptic selectivity filter binding site," Nature, vol. 547, pp. 364-368, 2017.

[111] C. McClenaghan, M. Schewe, P. Aryal, E. P. Carpenter, T. Baukrowitz, and S. J. Tucker, "Polymodal activation of the TREK-2 K2P channel produces structurally distinct open states," J. Gen. Physiol., vol. 147, no. 6, pp. 497505, 2016.

[112] D. S. Goodsell and A. J. Olson, "Structural symmetry and protein function," Annual review of biophysics and biomolecular structure, vol. 29, 2000.

[113] I. André, C. E. Strauss, D. B. Kaplan, P. Bradley, and D. Baker, "Emergence of symmetry in homooligomeric biological assemblies," Proceedings of the National Academy of Sciences, vol. 105, no. 42, pp. 16148-16152, 2008. 
[114] T. L. Blundell and N. Srinivasan, "Symmetry, stability, and dynamics of multidomain and multicomponent protein systems," Proceedings of the National Academy of Sciences, vol. 93, no. 25, pp. 14243-14248, 1996.

[115] W. R. Taylor, A. C. May, N. P. Brown, and A. Aszódi, "Protein structure: geometry, topology and classification," Reports on Progress in Physics, vol. 64, no. 4, p. 517, 2001.

[116] I. M. Klotz, N. Langebman, and D. Dahnall, "Quaternary structure of proteins," Annual review of biochemistry, vol. 39, no. 1, pp. 25-62, 1970.

[117] A. J. Cornish-Bowden and D. Koshland, "The quaternary structure of proteins composed of identical subunits," Journal of Biological Chemistry, vol. 246, no. 10, pp. 3092-3102, 1971.

[118] P. G. Wolynes, "Symmetry and the energy landscapes of biomolecules," Proceedings of the National Academy of Sciences of the United States of America, vol. 93, no. 25, p. 14249, 1996.

[119] J. P. Abrahams, A. G. Leslie, R. Lutter, and J. E. Walker, "Structure at 2.8 a resolution of f1-atpase from bovine heart mitochondria," Nature, vol. 370, no. 6491, p. 621, 1994.

[120] J. Monod, J. Wyman, and J. Changeux, "On the nature of allosteric transitions: A plausible model.," Journal of molecular biology, vol. 12, pp. 88-118, 1965 .

[121] D. Koshland Jr, G. Némethy, and D. Filmer, "Comparison of experimental binding data and theoretical models in proteins containing subunits," Biochemistry, vol. 5, no. 1, pp. 365-385, 1966.

[122] H. Saibil, D. Zheng, A. Roseman, A. Hunter, G. Watson, S. Chen, A. Auf der Mauer, B. O'hara, S. Wood, N. Mann, et al., "Atp induces large quaternary rearrangements in a cage-like chaperonin structure," Current Biology, vol. 3, no. 5, pp. 265-273, 1993.

[123] R. Pfoh, A. Li, N. Chakrabarti, J. Payandeh, R. Pomès, and E. F. Pai, "Structural asymmetry in the magnesium channel cora points to sequential allosteric regulation," Proceedings of the National Academy of Sciences, vol. 109, no. 46, pp. 18809-18814, 2012.

[124] D. Matthies, O. Dalmas, M. J. Borgnia, P. K. Dominik, A. Merk, P. Rao, B. G. Reddy, S. Islam, A. Bartesaghi, E. Perozo, et al., "Cryo-em structures of the magnesium channel cora reveal symmetry break upon gating," Cell, vol. 164 , no. 4, pp. 747-756, 2016. 
[125] M. Bonjack-Shterengartz and D. Avnir, "The near-symmetry of proteins," Proteins: Structure, Function, and Bioinformatics, vol. 83, no. 4, pp. 722734, 2015.

[126] H. Zabrodsky, S. Peleg, and D. Avnir, "Continuous symmetry measures," Journal of the American Chemical Society, vol. 114, no. 20, pp. 7843-7851, 1992.

[127] M. Pinsky, C. Dryzun, D. Casanova, P. Alemany, and D. Avnir, "Analytical methods for calculating continuous symmetry measures and the chirality measure," Journal of computational chemistry, vol. 29, no. 16, pp. 2712 2721, 2008.

[128] M. Bonjack-Shterengartz and D. Avnir, "The enigma of the near-symmetry of proteins: Domain swapping," PloS one, vol. 12, no. 7, p. e0180030, 2017.

[129] J. T. Brennecke and B. L. de Groot, "Mechanism of mechanosensitive gating of the trek-2 potassium channel," Biophysical journal, vol. 114, no. 6, pp. 1336-1343, 2018.

[130] M. Haupt, M. P. Blakeley, S. J. Fisher, S. A. Mason, J. B. Cooper, E. P. Mitchell, and V. T. Forsyth, "Binding site asymmetry in human transthyretin: insights from a joint neutron and x-ray crystallographic analysis using perdeuterated protein," IUCrJ, vol. 1, no. 6, pp. 429-438, 2014.

[131] R. B. Best, G. Hummer, and W. A. Eaton, "Native contacts determine protein folding mechanisms in atomistic simulations," Proceedings of the $\mathrm{Na}$ tional Academy of Sciences, vol. 110, no. 44, pp. 17874-17879, 2013.

[132] J. Hamilton and M. Benson, "Transthyretin: a review from a structural perspective," Cellular and Molecular Life Sciences CMLS, vol. 58, no. 10, pp. 1491-1521, 2001.

[133] G. Schreiber, B. Southwell, and S. Richardson, "Hormone delivery systems to the brain-transthyretin," Experimental and Clinical Endocrinology $\& 5$ Dabetes, vol. 103, no. 02, pp. 75-80, 1995.

[134] S. A. Bernhard and R. A. MacQuarrie, "Half-site reactivity and the "inducedfit" hypothesis," Journal of molecular biology, vol. 74, no. 1, pp. 73-78, 1973.

[135] F. Seydoux, O. Malhotra, S. Bernhard, and G. Stark, "Half-site reactivit," CRC critical reviews in biochemistry, vol. 2, no. 2, pp. 227-257, 1974. 
[136] W. B. Stallcup and D. Koshland Jr, "Half-of-the-sites reactivity and negative co-operativity: The case of yeast glyceraldehyde 3-phosphate dehydrogenase," Journal of molecular biology, vol. 80, no. 1, pp. 41-62, 1973.

[137] N. N. K, "Interdomain interactions in oligomeric enzymes: creation of asymmetry in homo-oligomers and role in metabolite channeling between active centers of hetero-oligomers," FEBS Letters, vol. 487, no. 3, pp. 327-332, 2001. 


\section{Appendix A}

\section{Supplementary Material Asymmetry}

In the following a user manual on usage of the algorithms for asymmetry quantification as presented in chapter 7 is given, followed by additional figures. Both are submitted for publication as supplementary material to the putative publication of chapter 7 .

\section{A.1 User Manual Asympy}

This manual provides detailed information on the usage of the provided tool. The package can be downloaded from https:github.com/jtbrennecke/asympy. To run the programs, Python 2.7 with these packages is required:

- pylab

- mdtraj

- scipy

- optparse

- sklearn

- ipy_progressbar (optional)

First, the usage of the CSM calculation tool (CSM.py) with the individual subunit contributions is introduced. In the next part, the usage of the tool to calculate the symmetry measure according to the FAME algorithm (FAME.py) is demonstrated. To be able to calculate the FAME algorithm correctly, the trajectory and the functional input data have to be prepared to get a symmetric reference motion (see figure A.5). The preparation is done using the prepare_files.py tool.

For all examples provided, various files are used. All tools require a PDB structure 
file (struct.pdb), which has to be matched with a trajectory file containing an ensemble of structures, e.g. a MD trajectory (traj.xtc). The CSM and the preparative tools expect the sorting of the subunits to be either clockwise or counter-clockwise. However, if the sorting differs from this convention, it can be changed by giving the atom ranges of each subunit in (counter-)clockwise order. A tetramer with a clockwise labeling of the subunits $1,3,2$, 4 with 10 atoms each could be analyzed using the parameter setting -c' $[[0,10],[20,30],[10,20],[30,40]]$ '.

\section{CSM.py}

By the CSM tool the asymmetry for each frame of a trajectory is calculated:

CSM.py -f traj.xtc -s struct.pdb -n 4 -o asym.txt -e asym.xtc.

This command takes a trajectory with a matching PDB file with four subunits $(-n$ 4) and calculates the CSM measure. This measure in combination with the measure for the individual subunits is written to the file asym.txt. A typical output is shown in table A.1. Here the first column is the frame number. The second

$$
\begin{array}{cccccc}
\text { \# } & \text { Frame } & \text { CSM } & \text { overall, CSM } & \text { for } & \text { subunits } \\
0 & 0.95 & 0.24 & 0.45 & 0.12 & 0.14 \\
1 & 1.2 & 0.32 & 0.47 & 0.2 & 0.21
\end{array}
$$

Table A.1.: asym.txt Example output of CSM tool.

column gives the overall CSM score. The columns starting from the third column are the partial CSM scores for the different subunits.

An additional result from the CSM calculation is the fully symmetric structure for each frame. These structures are saved into the asym.xtc file.

\section{prepare_files.py}

The prepare_files program prepares the input for the FAME algorithm. As described in the main manuscript, a symmetrized input is needed to create a symmetric PLS-FMA vector:

prepare files.py -f traj.xtc -s struct.pdb - n 4 -d data.dat -x out.xtc -p out.dat.

This command takes the data file data.dat, the format should be similar to the example input in table A.2, and the trajectory file. As a result, the extended and rotated trajectory according to figure A.5 is written to $-x$ out.xtc. The input data is copied into the output file - $p$ out.dat to match the trajectory for the FAME tool. 


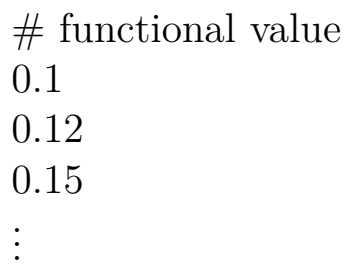

Table A.2.: data.dat format for the input data file. Alternatively, the frame number can be given before the functional value.

\section{FAME.py}

After preparing the input files using the prepare_files program, the output can be used to run the FAME algorithm. For the FAME algorithm, the optimal number of PLS components have to be determined first to avoid overfitting. The determination is done by running the FAME program with a negative value for the number of PLS components, that tests components starting at one to the absolute of the given number:

FAME.py -f out.xtc -s struct.pdb -n 4 -d out.dat -c -10 -o comp.dat.

The command tries components one to ten and evaluates the correlation. The program automatically uses the first half of the input as training data and the second half of the data as cross-validation data. An example output (-o comp.dat) is illustrated in figure A.3. In this example, three components would be a good choice.

Consequently, the FAME program can be run with three PLS components:

FAME.py -f out.xtc -s struct.pdb -n 4 -d out.dat -c 3 -w model.dat -o contributions.dat

-e extremes_ew.pdb-p extremes.pdb.

As an input for FAME, the output files of the prepare files program are used. The output is the prediction for the functional value $\tilde{f}(-w$ model.dat). To get an idea on which parts are predicted well and which parts are not, the prediction $\tilde{f}$ can be visualized with the input data $f$ (see figure A.5). Note that for the final FAME algorithm the data set is not split into training and cross-validation data blocks but all data is used to build an optimal model.

Furthermore, the contributions $C_{j}$ for each of the subunits are written to -o contributions.dat. The PDB file - $p$ extremes.pdb gives a graphical representation of the reference motion and -e extremes_ew.pdb gives the ensemble weighted representation of the motion (refer to Hub and de Groot for further explanation).

The PLS-FMA algorithm is insensitive to the absolute values of the functional 
input data. However, for the decomposition of the PLS-FMA prediction into the subunit components and especially for the contribution calculation, the selecteion of the absolute values of the functional input is crucial. As $\underline{C}_{j}=\underline{\tilde{f}}_{j} / \underline{\tilde{f}}$ it is clear that changing the sign in $\underline{\tilde{f}}_{j}$ or $\underline{\tilde{f}}$ would require the other value to change its sign accordingly to keep $\underline{C}_{j}$ in the range of $0 \leq \underline{C}_{j} \leq 1$, which is required for this measure. Thus applying a linear transformation on the functional input data $(f)$ might be required to get a reasonable prediction for the contributions. The algorithm would apply no value to $\underline{C}_{j}$ if it is outside the range. Therefore, the number of missing values combined with the expected continuity of the contributions $\underline{C}_{j}$ (sudden changes in asymmetry are not expected for a continuous simulation) can be used to estimate the need for a linear transformation. If a linear transformation is applied, the missing values can be used to evaluate the improvement of the results through the transformation.

Note that the current implementation reassigns subunit labels and minimizes the RMSD to a given reference to achieve symmetry correction. However, an alternative (and mathematically more correct but computationally significantly more demanding) method is the calculation of the correct rotation axis followed by a rotation around this, similar to the CSM approach. (See figure A.4 as an example for TTR.) 


\section{A.2 Supplementary Figures}

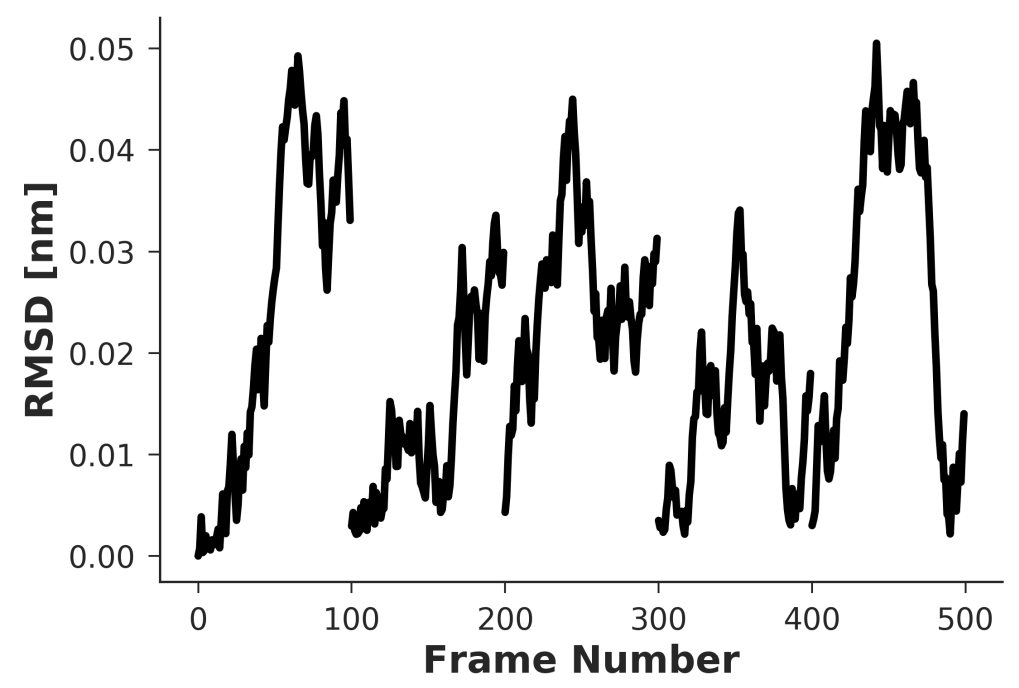

Figure A.1.: RMSD KcsA The RMSD of the trajectory of KcsA in reference to the symmetric crystal structure demonstrates how small the introduced asymmetric motions actually are.

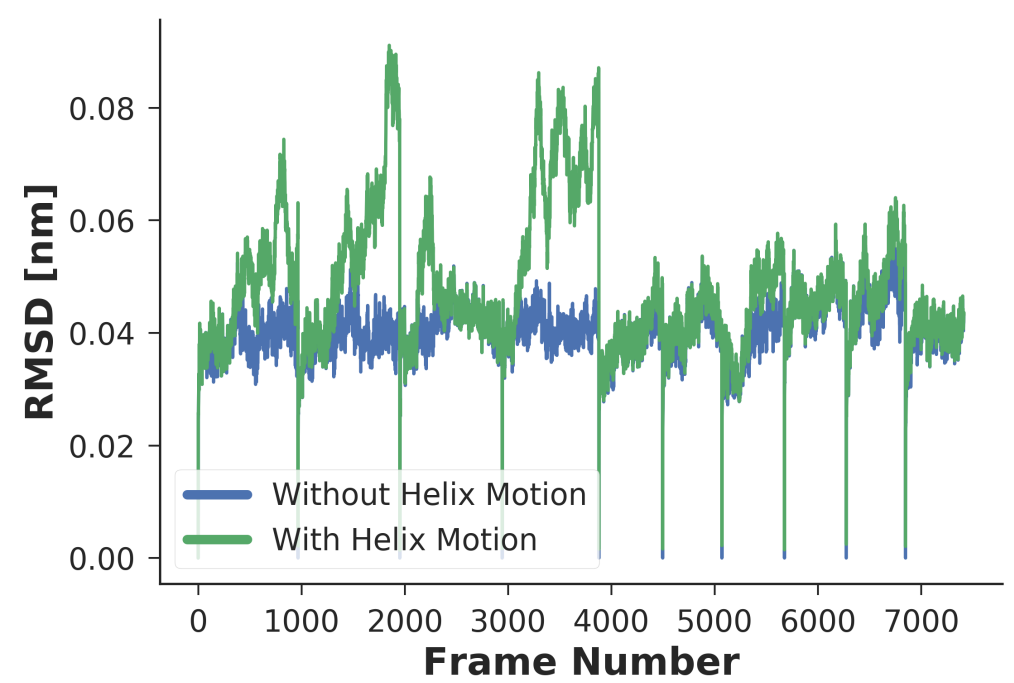

Figure A.2.: RMSD TREK-2 The RMSD of the trajectory of TREK-2 comparing the structure of the original trajectory (blue line) and the trajectory after introducing the helix motion (green line). 


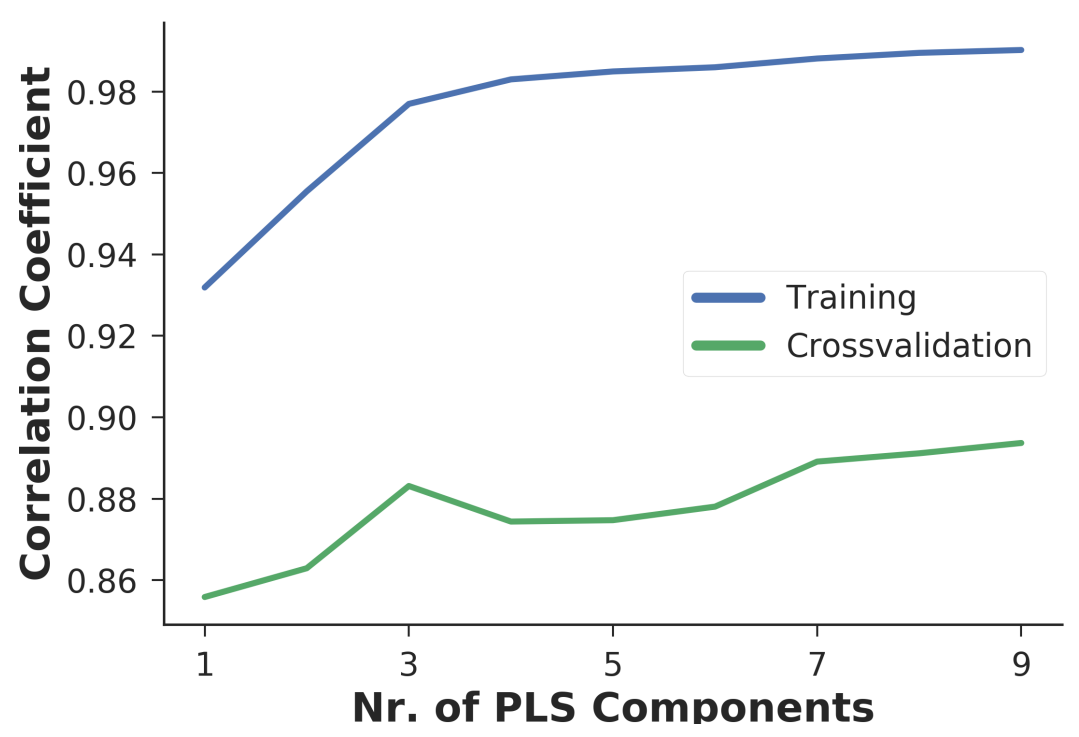

Figure A.3.: PLS component estimation To perform the PLS-FMA analysis, the correct number of PLS components has to be determined previously. Different PLS components have to be tested and the correlation between the functional input data and the model created with this number of components is calculated. The correlation for the training data is shown in blue and the correlation for the crossvalidation is shown in green.

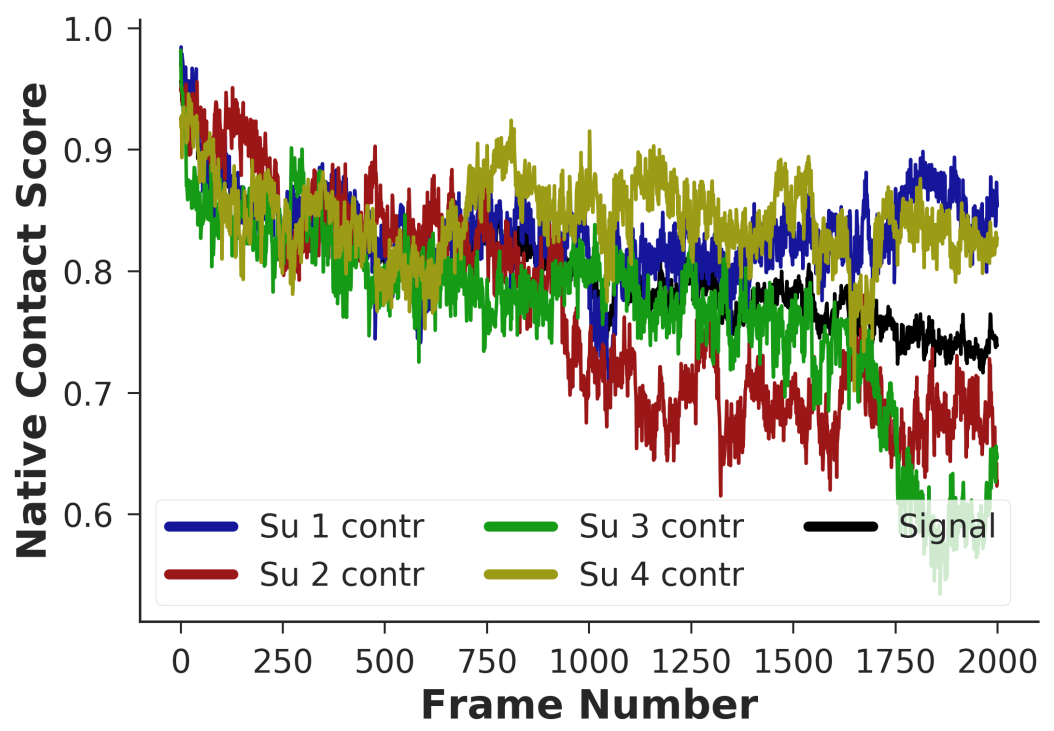

Figure A.4.: TTR contacts The black signal line in the background represents the overall contact score of the TTR simulations by calculating the native contact score based on the subunits independently (colored lines). 


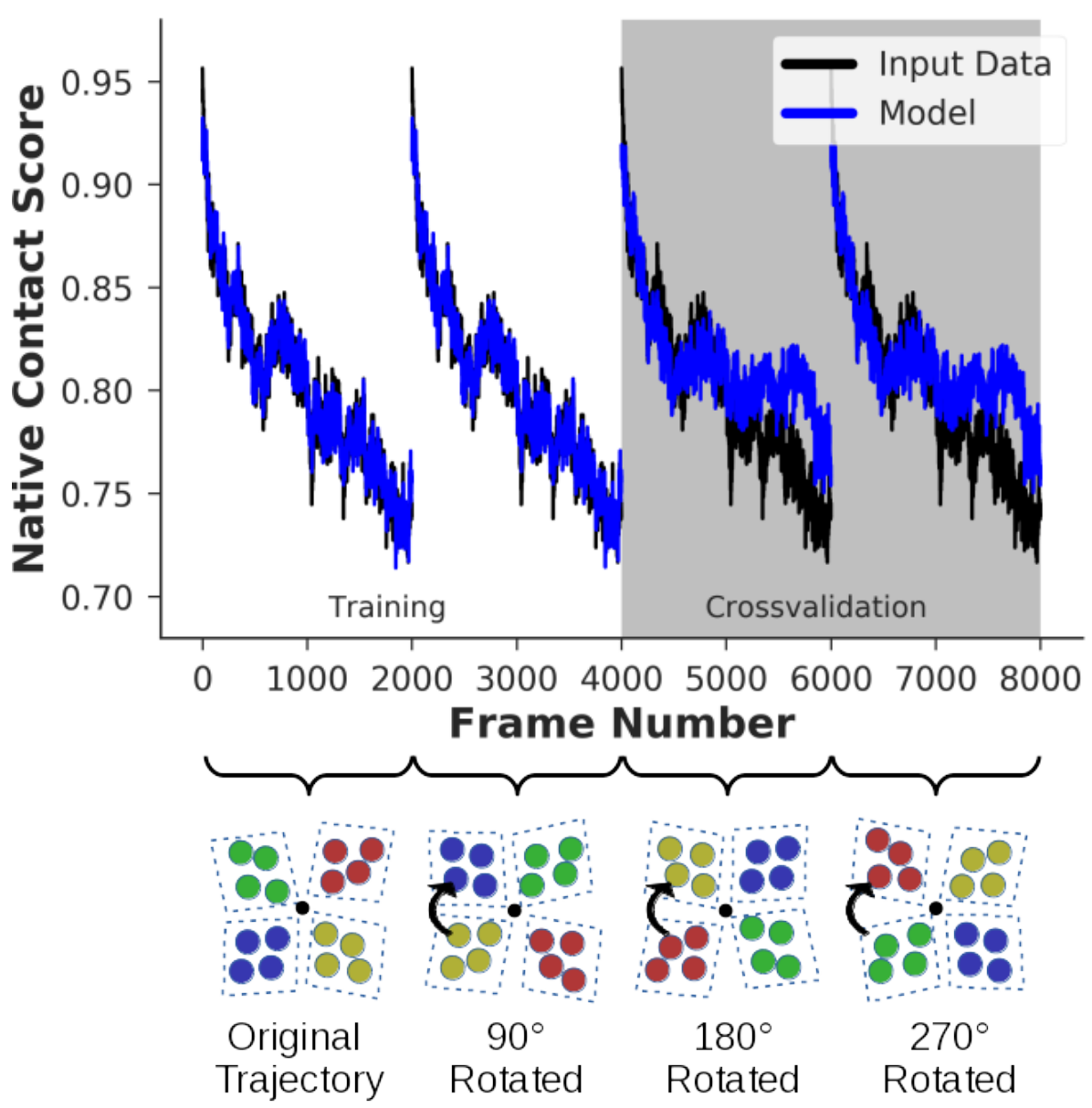

Figure A.5.: TTR rotation scheme To symmetrize the reference motion, the input ensemble is symmetrized by using all possible assignments of the labels. Thus the functional values are appended such that the overall dataset includes as many times the same functional value as subunits in the protein. Furthermore, the trajectory itself is duplicated by the same number. In the first repeat of the trajectory all subunits are rotated by 360 degrees divided by the number of subunits. The next repeat will be rotated by twice that and so on.

The input data (black line) is split in two halves where the first halve (white background) is used to train the model (blue line). For crossvalidation all input data are compared to the created model (gray background). 


\section{Curriculum Vitae}

\section{Personal}

Name: Julian Tim Brennecke

Address: Stargarder Weg 18, 37083 Göttingen, Germany

Birth date: 08.02.1991, Hildesheim, Germany

\section{Education}

03/2015 - present: PhD at the Max Planck Institute for Biophysical Chemistry in the group for Biomolecular Dynamics, Göttingen, Germany Supervisor Bert L. de Groot

04/2013 - 10/2014: M.Sc. in Physics at Georg-August-Universität Göttingen, Germany 10/2010 - 03/2014: B.Sc. In Physics at Georg-August-Universität Göttingen, Germany 08/2008 - 08/2010: Abitur Christian-von-Dohm-Gymnasium Goslar, Germany 